Pure and Applied Mathematics Quarterly

Volume 4, Number 3

(Special Issue: In honor of

Fedor Bogomolov, Part 2 of 2 )

$785-875,2008$

\title{
Non-commutative Hodge-to-de Rham Degeneration via the Method of Deligne-Illusie
}

\author{
Dmitry Kaledin ${ }^{1}$
}

To Fedor Bogomolov, with gratitude and appreciation

to celebrate his 60-th birthday.

\section{Contents}

$\begin{array}{ll}\text { Introduction } & 786\end{array}$

$\begin{array}{ll}\text { Acknowledgements. } & 789\end{array}$

$\begin{array}{ll}\text { Note added in proof. } & 790\end{array}$

$\begin{array}{ll}\text { 1. Linear algebra. } & 790\end{array}$

1.1. Recollection on homology of small categories. 790

1.2. Example: simplicial vector spaces. 793

1.3. Effectively finite simplicial objects. $\quad 796$

$\begin{array}{lll}\text { 1.4. Fibrations and cofibrations. } & 798\end{array}$

1.5. Versions of the cyclic category. 802

1.6. Cyclic homology and periodicity. 804

1.7. Homological effects of the $p$-fold coverings. 808

1.8. Relation to group homology. 811

Received December 1, 2006.

${ }^{1}$ Partially supported by CRDF grant RUM1-2694. 
2. Cartier map - the simple case. 813

2.1. Cyclic homology of algebras. 813

2.2. Polycyclic objects and the inverse Cartier map. 816

3. Algebraic topology. 820

3.1. Generalities on stable homotopy. $\quad 820$

3.2. Additivization. 826

3.3. The cube construction. 834

3.4. Multiplication. $\quad 839$

4. Cartier map - the general case. 843

4.1. Generalized Cartier map. 843

$\begin{array}{ll}\text { 4.2. Splitting at first order. } & 848\end{array}$

4.3. Splitting in higher orders. 855

5. Degeneration. 861

5.1. Statements. 861

5.2. Cosimplicial algebras and splittings. 864

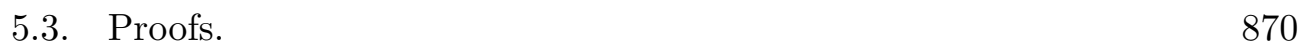

$\begin{array}{ll}\text { References } & 873\end{array}$

\section{INTRODUCTION}

Hodge theory, since its appearance in complex analysis, has long become one of the most useful tools in complex algebraic geometry. At the core of its algebraic applications lies a statement first extracted by P. Deligne [D2]; this statement in itself is purely algebraic. It claims that for a smooth projective algebraic variety $X$ over a field $K$ of characteristic 0 , the natural spectral sequence

$$
H^{p}\left(X, \Omega_{X}^{q}\right) \Rightarrow H_{D R}^{p+q}(X)
$$

degenerates at first term, so that we have a non-canonical decomposition $H_{D R}^{p+q}(X)$ $=\bigoplus H^{p}\left(X, \Omega_{X}^{q}\right)$. Here $H_{D R}^{\cdot}(X)$ is the de Rham cohomology of $X$, - that is, 
the hypercohomology of $X$ with coefficients in the de Rham complex, - and $H^{\bullet}\left(X, \Omega_{X}^{*}\right)$ is its cohomology with coefficients in the coherent sheaves of differential forms (these days known as Hodge cohomology). The spectral sequence is known as the Hodge-to-de Rham spectral sequence.

The degeneration statement has two proofs. One is analytic and gives much more (including a canonical form of the decomposition $H_{D R}^{p+q}(X)=\bigoplus H^{p}\left(X, \Omega_{X}^{q}\right)$ ). The other is purely algebraic and gives exactly the degeneration. This proof has been discovered by P. Deligne and L. Illusie [DI], following earlier work by G. Faltings; it is based on reduction to positive characteristic.

It has been known for some time now that all the terms in the Hodge-to-de Rham spectral sequence can be defined naturally in much greater generality: instead of a scheme $X$, one can consider a site, or a topological space, equipped with a sheaf of non-commutative associative algebras $\mathcal{A}$. In this generalization, the role of differential forms is played by the Hochschild homology sheaves $H_{\bullet}(\mathcal{A})$, and the de Rham complex is replaced with the cyclic homology complex $H C$. $(\mathcal{A})$ discovered in about 1982 independently by A. Connes [Co], J.-L. Loday and D. Quillen [LQ], and B. Feigin and B. Tsygan [FT1].

Thus the Hodge-to-de Rham spectral sequence is well-defined for non-commutative algebras, and a natural question to ask is when does it degenerate. Somewhat surprisingly, it seems that this did not receive much attention for a long time. Recently, things have changed. There was a resurgence of interest in "non-commutative geometry", motivated in part by physical applications. In particular, we refer the reader to a large paper [KS] by M. Kontsevich and Y. Soibelman. Among many other things, they give a precise conjectural form of the Hodge-to-de Rham degeneration statement in the non-commutative world.

The goal of this paper is to prove the Kontsevich-Soibelman Conjecture (unfortunately, under some additional assumptions). To do this, we use the method of Deligne-Illusie.

To an algebraic geometer, this would at first seem hopeless, since the DeligneIllusie method relies on the Cartier isomorphism between de Rham cohomology classes and differential forms in positive characteristic, which in turn comes from the Frobenius map. The Frobenius map obviously has no non-commutative counterpart. Thus the author was very surprised when he realized in 2005 that the 
Cartier map is, nevertheless, perfectly well defined in the non-commutative world, and one can apply the argument in [DI] with minimal modifications. This has been done in a way in the preprint [Ka], where a form of non-commutative Hodgeto-de Rham degeneration was proved.

Since then, the author had an opportunity to understand things better, and to discuss the subject with many people. It turned out that the non-commutative Cartier map, while a surprise to almost all algebraic geometers, is practically a banality for algebraic topologists. Namely, in the framework of the so-called Topological Cyclic Homology introduced by M. Bökstedt, W.C. Hsiang and I. Madsen $[\mathrm{BHM}],[\mathrm{M}]$, the Cartier map appears at least implicitly, as a part of the notion of a cyclotomic spectrum, and actually quite explicitly - in particular, in the paper $[\mathrm{H}]$ of L. Hesselholt, where the author constructs a version of Witt vectors for non-commutative rings. We recommend this very clear paper, written apparently with an eye to a non-topologist reader, as an entrance point to the subject for people not familiar with topological language; a nice introduction can also be found in $[\mathrm{HM}]$. In fact, it seems that the degeneration statement itself was not discovered ten years ago only by a freak accident.

Thus ideologically, the subject naturally belongs to algebraic topology, and even in $[\mathrm{Ka}]$, the topological flavor is quite clear. On the other hand, there is a definite difference of language between algebraic topology and algebraic geometry, probably responsible for the break in communications mentioned above. In Topological Cyclic Homology, the standard language is that of equivariant stable homotopy theory. In author's experience, algebraic geometers are quite comfortable with homological algebra and can accept on trust some stable homotopy; but the complicated machinery of equivariant spectra is beyond reach for most (sadly, including the author).

Since for us, the main interest of Hodge-to-de Rham degeneration lies in applications to algebraic geometry, be it commutative or not, we have decided to attempt a certain compomise. While we try to fully acknowledge the topological origin of most of our constructions and explain it at least informally, formally, the paper is completely independent from topological notions. In particular, the generalized Cartier map that we eventually construct is a map of complexes, not a map of spectra. Thus everything should be accessible to an algebraic geometer who does not even know the definition of a spectrum. To an algebraic 
topologist, many pages of the paper would seem hopelessly naive, for which we can only apologize. Same goes for possibly inaccurate historical references and misattributions.

The paper is largely self-contained. It is organized as follows. In Section 1, we give the necessary preliminaries - this includes generalities on homology of small categories, basic facts on cyclic homology (mostly within the scope of [L]), and also some facts about the relation between periodic cyclic homology and Tate homology of cyclic groups. In Section 2, we introduce cyclic homology of algebras, and we construct the Cartier map under one additional technical assumption. This assumption is very strong; thus in practice, the Cartier map defined in Section 2 is almost useless. However, we feel that it would be better to first explain things in a simple special case. Section 3 is the technical heart of the paper: in Subsection 3.1, we explain informally the relation of our constructions with those known in algebraic topology, and in the rest of the Section, which is formally independent from Subsection 3.1, we give these constructions. We note that of crucial importance here are some techniques that we have learned from the work of T. Pirashvili; essentially, it is these techniques which allow one to stay within homological algebra to the end, without formal references to topological notions. It should be stressed that very little, perhaps nothing at all in Section 3 is new (but there are no references in a form convenient for our applications). In Section 4, we use Section 3 to define a generalized Cartier map, and then show under what additional conditions it can be stripped down to an isomorphism, as required for the Deligne-Illusie method. Finally, in Section 5 we discuss various degeneration statements, formulate the Kontsevich-Soibelman Conjecture, and prove it under additional assumptions.

Acknowledgements. Throughout this work, continuous attention and encouragement of A. Beilinson, M. Kontsevich, L. Katzarkov and N. Markarian were extremely helpful; I have benefitted much from discussions with them. After [Ka] was posted, I had an opportunity to meet L. Hesselholt. I am very grateful for his interest, explanations, and answers to all of my questions, which I am sure were often naive to the point of trying one's patience. I am also very grateful to M. Bershtein, R. Bezrukavnikov, A. Braverman, B. Feigin, V. Franjou, E. Getzler, D. Kazhdan, A. Khoroshkin, A. Kuznetsov, S. Loktev, A. Losev, G. Merzon, T. 
Pantev, G. Sharygin, D. Tamarkin, B. Tsygan, M. Verbitsky, and V. Vologodsky for helpful discussions. A crucial part of this work was done while visiting KIAS in Seoul in the beautiful springtime of 2006, at a kind invitation of Bumsig Kim. Conjecture 5.4 owes much to discussions with B. Toën and B. Keller (in fact, it might properly be attributed to B. Toën).

The paper is somewhat long and somewhat technical, and it has required a lot of editing. Sasha Kuznetsov volunteered to help, and he did an absolutely fantastic job of reading a first draft of the paper, indicating numerous gaps and inaccuracies to me, and suggesting many improvements in the exposition.

I am thankful to the referee for some important comments and for indicating some gaps.

Finally, it is a great pleasure to dedicate the paper to Fedya Bogomolov, who turns 60 this year. His continuous and discrete presense in Russian mathematics in particular, and in Mathematics as a whole, has always been a great source of inspiration for me. I hope this paper amuzes him. In fact, I am quite surprised it contains no references to his work, since all my previous papers did, no matter the choice of a subject. I strongly suspect this is only due to my ignorance the relevant Bogomolov Theorem, yet another one, probably has been proved 30 years ago and just somehow escaped my attention.

Note added in proof. At about the same time as the first version of this paper was posted to arxiv.org, B. Toën gave a very elegant and short proof of what was Conjecture 5.4 and is now Theorem 5.4. This allows us to get rid of the most restrictive assumption in the first version of our Theorem 5.5.

\section{Linear ALgebra.}

1.1. Recollection on homology of small categories. In our approach to cyclic homology, we follow [Co] and use the toolkit of homology of small categories (for more detailed expositions of this point of view, see [L, Section 6] or [FT2]). For the convenience of the reader, let us recall what forms this toolkit.

Fix a base field $k$. For any small category $\Gamma$, denote by $\operatorname{Fun}(\Gamma, k)$ the category of functors from $\Gamma$ to the category $k$-Vect of $k$-vector spaces. This is an abelian category with enough injectives and enough projectives; we denote the 
corresponding derived category by $\mathcal{D}(\Gamma, k)$ (or $\mathcal{D}^{b}(\Gamma, k), \mathcal{D}^{+}(\Gamma, k), \mathcal{D}^{-}(\Gamma, k)$, as the needs arise).

One can look at the category $\operatorname{Fun}(\Gamma, k)$ in two complementary ways. Algebraically, one thinks of a small category as a generalization of a group, so that $\operatorname{Fun}(\Gamma, k)$ is a generalization of the category of representations of a group. Once the base field $k$ is fixed, a representation of a group $G$ is the same thing as a module over its group algebra $k[G]$; analogously, one can treat objects in $\operatorname{Fun}(\Gamma, k)$ as modules over an "algebra with many objects".

Topologically, $\operatorname{Fun}(\Gamma, k)$ is the same thing as the category of presheaves of $k$ vector spaces on the opposite category $\Gamma^{o}$; in many respects, objects in $\operatorname{Fun}(\Gamma, k)$ behave in the same way as usual sheaves on topological spaces.

In particular, the category $\operatorname{Fun}(\Gamma, k)$ is a symmetric tensor category (with pointwise tensor product, $(F \otimes G)([a])=F([a]) \otimes G([a])$ for any object $[a] \in \Gamma$ and any $F, G \in \operatorname{Fun}(\Gamma, k))$. We also have an exterior tensor product: for any two small categories $\Gamma^{\prime}, \Gamma$, and any $F \in \operatorname{Fun}(\Gamma, k), F^{\prime} \in \operatorname{Fun}\left(\Gamma^{\prime}, k\right)$, we define $F \otimes F^{\prime} \in \operatorname{Fun}\left(\Gamma \times \Gamma^{\prime}, k\right)$ by $\left(F \otimes F^{\prime}\right)\left([a] \times\left[a^{\prime}\right]\right)=F([a]) \otimes F^{\prime}\left(\left[a^{\prime}\right]\right)$. For any functor $f: \Gamma^{\prime} \rightarrow \Gamma$ between small categories $\Gamma, \Gamma^{\prime}$, we have the restriction functor $f^{*}: \operatorname{Fun}(\Gamma, k) \rightarrow \operatorname{Fun}\left(\Gamma^{\prime}, k\right)$. It has a right-adjoint $f_{*}: \operatorname{Fun}\left(\Gamma^{\prime}, k\right) \rightarrow \operatorname{Fun}(\Gamma, k)$ and a left-adjoint $f_{!}: \operatorname{Fun}\left(\Gamma^{\prime}, k\right) \rightarrow \operatorname{Fun}(\Gamma, k)$; in category theory, these are known as the Kan extensions. If $f: \Gamma^{\prime} \rightarrow \Gamma$ admits a left-adjoint $f^{\prime}: \Gamma \rightarrow \Gamma^{\prime}$, then $f^{*}$ is left-adjoint to $f^{\prime *}$, so $f^{*} \cong f_{!}^{\prime}$ (and similarly for right-adjoints). In the particular case when $f=\iota_{[a]}: \mathrm{pt} \rightarrow \Gamma$ is the embedding of an object $[a] \in \Gamma$, we obtain functors $\iota_{[a] !} k, \iota_{[a] *} k$, which are called the functors represented and corepresented by $[a]$. Explicitly, for any $[b] \in \Gamma$ we have

$$
\left(\iota_{[a] !} k\right)([b])=k[\Gamma([a],[b])], \quad\left(\iota_{[a] *} k\right)([b])=k[\Gamma([b],[a])]^{*},
$$

the $k$-vector space spanned by the sets of maps in $\Gamma$ from $[a]$ to $[b]$ and the dual to the $k$-vector space spanned by the set of maps from $[b]$ to $[a]$. Representable functors are projective, and corepresentable functors are injective; both generate the category $\operatorname{Fun}(\Gamma, k)$. The functor $f_{*}$ is exact on the left, and the functor $f_{!}$is exact on the right, so that we can form derived functors $L^{\bullet} f_{!}: \mathcal{D}^{-}\left(\Gamma^{\prime}, k\right) \rightarrow \mathcal{D}^{-}(\Gamma, k)$, $R^{\bullet} f_{*}: \mathcal{D}^{+}\left(\Gamma^{\prime}, k\right) \rightarrow \mathcal{D}^{+}(\Gamma, k)$. In the particular case when $f=\tau: \Gamma \rightarrow$ pt is the projection to a point pt, this gives the notions of homology and cohomology of the 
small category $\Gamma$ with coefficients in some functor $E \in \operatorname{Fun}(\Gamma, k)$ :

$$
H_{\bullet}(\Gamma, E)=L^{\bullet} \tau_{!} E, \quad H^{\bullet}(\Gamma, E)=R^{\bullet} \tau_{*} E .
$$

For any two small categories $\Gamma, \Gamma^{\prime}$, and any $F \in \operatorname{Fun}(\Gamma, k), F^{\prime} \in \operatorname{Fun}\left(\Gamma^{\prime}, k\right)$, we have the Künneth formula

$$
H_{.}\left(\Gamma \times \Gamma^{\prime}, F \otimes F^{\prime}\right) \cong H_{\bullet}(\Gamma, F) \otimes H_{\bullet}\left(\Gamma^{\prime}, F^{\prime}\right) .
$$

By abuse of notation, we will denote by $k \in \operatorname{Fun}(\Gamma, k)$ the constant functor which sends every object in $\Gamma$ to $k$ (properly speaking, this should be denoted $\tau^{*} k$ ). Just as in the case of sheaves on a topological space, for any $E \in \operatorname{Fun}(\Gamma, k)$ we have

$$
H^{\bullet}(\Gamma, E) \cong \operatorname{Ext}_{\mathrm{Fun}(\Gamma, k)}^{\bullet}(k, E)
$$

In particular, the cohomology $H^{\bullet}(\Gamma, k)=\operatorname{Ext}_{\mathrm{Fun}(\Gamma, k)}(k, k)$ is an algebra, and for any $E \in \operatorname{Fun}(\Gamma, E), H^{*}(\Gamma, k)$ is a module over the algebra $H^{*}(\Gamma, k)$. So is the homology $H_{\bullet}(\Gamma, E)$. To interpret homology in a way similar to (1.1), one can use the algebraic interpretation of $\operatorname{Fun}(\Gamma, k)$. Namely, for any $E \in \operatorname{Fun}(\Gamma, k)$, $F \in \operatorname{Fun}\left(\Gamma^{o}, k\right)$, one defines the "convolution" tensor product $F \otimes_{\Gamma} E$ as the cokernel of the natural map

$$
\bigoplus_{\substack{f:[a] \rightarrow[b],[a],[b] \in \Gamma}} F([b]) \otimes E([a]) \stackrel{F(f) \otimes \mathrm{id}-\mathrm{id} \otimes E(f)}{\longrightarrow} \bigoplus_{[a] \in \Gamma} F([a]) \otimes E([a]),
$$

where the sum on the left-hand side is taken over all morphisms in $\Gamma$, and the sum on the right-hand side is taken over all objects. In category theory, this construction is known as "coend"; it is completely analogous to the tensor product of a left module and a right module over an associative algebra. In particular, convolution is right-exact. For any $E \in \operatorname{Fun}(\Gamma, k)$, we have

$$
H_{\text {. }}(\Gamma, E) \cong \operatorname{Tor}_{\Gamma}^{\cdot}(k, E),
$$

where $\operatorname{Tor}_{\Gamma}{ }_{\Gamma}$ are the derived functors of the convolution functor $\otimes_{\Gamma}$. We also have a version of the projection formula: given a functor $\tau: \Gamma^{\prime} \rightarrow \Gamma$ and functors $F \in \operatorname{Fun}(\Gamma, k), G \in \operatorname{Fun}\left(\Gamma^{\prime o}, k\right)$, we have a natural isomorphism

$$
F \otimes_{\Gamma} \tau_{!}^{o} G \cong \tau^{*} F \otimes_{\Gamma^{\prime}} G,
$$

and similarly for derived functors $L^{\bullet} \tau_{!}$, Tor $\dot{\Gamma}_{\Gamma}$. For any $F^{\prime} \in \operatorname{Fun}\left(\Gamma^{\prime}, k\right), G \in$ $\operatorname{Fun}\left(\Gamma^{\prime o}, k\right)$, we can set $F=\tau_{!} F^{\prime}$; then by adjunction, we have a natural map 
$F^{\prime} \rightarrow \tau^{*} F$, and (1.4) induces a natural map

$$
F^{\prime} \otimes_{\Gamma^{\prime}} G \rightarrow \tau^{*} F \otimes_{\Gamma^{\prime}} G \cong F \otimes_{\Gamma} \tau_{!}^{o} G \cong \tau_{!} F^{\prime} \otimes_{\Gamma} \tau_{!}^{o} G
$$

(and similarly for the derived functors). The convolution tensor product $\otimes_{\Gamma}$ is sufficiently functorial, so that, given small categories $\Gamma, \Gamma_{1}, \Gamma_{2}$ and functors $E_{1} \in$ $\operatorname{Fun}\left(\Gamma_{1} \times \Gamma, k\right), E_{2} \in \operatorname{Fun}\left(\Gamma^{o} \times \Gamma_{2}, k\right)$, we can define $E_{1} \otimes_{\Gamma} E_{2} \in \operatorname{Fun}\left(\Gamma_{1} \times \Gamma_{2}, k\right)$ by applying (1.2) pointwise, for every object $\left[a_{1}\right] \times\left[a_{2}\right] \in \Gamma_{1} \times \Gamma_{2}$. The same is true for the derived functors $\operatorname{Tor}_{\Gamma}^{\cdot}(-,-)$. A useful application of this technique is the following: for any two small categories $\Gamma, \Gamma^{\prime}$, a functor $K \in \operatorname{Fun}\left(\Gamma^{o} \times \Gamma^{\prime}, k\right)$ defines a functor

$$
E \mapsto E \otimes_{\Gamma} K
$$

from $\operatorname{Fun}(\Gamma, k)$ to $\operatorname{Fun}\left(\Gamma^{\prime}, k\right)$. In such a situation, we will say that the functor is represented by the kernel $K \in \operatorname{Fun}\left(\Gamma^{o} \times \Gamma^{\prime}, k\right)$.

1.2. Example: simplicial vector spaces. Let $\Gamma=\Delta^{o}$, the opposite to the category of finite non-empty linearly ordered sets. Then $\operatorname{Fun}\left(\Delta^{o}, k\right)$ is the category of simplicial $k$-vector spaces $E$. Since $\Delta^{o}$ has an initial object, the constant functor $k \in \operatorname{Fun}\left(\Delta^{o}, k\right)$ is representable, hence projective; therefore for any simplicial $k$-vector space $E_{.} \in \operatorname{Fun}\left(\Delta^{o}, k\right)$, we have $H^{\geq 1}\left(\Delta^{o}, E_{\mathbf{0}}\right)=0$. By Yoneda Lemma, $H^{0}\left(\Delta^{o}, E_{\bullet}\right)=E_{0}$. On the other hand, the homology $H_{\bullet}\left(\Delta^{o}, E\right)$ is usually non-trivial.

Lemma 1.1. For any simplicial $k$-vector space $E . \in \operatorname{Fun}\left(\Delta^{o}, k\right)$, the homology $H_{.}\left(\Delta^{o}, E_{\bullet}\right)$ can be computed by the standard complex $\left\langle E_{\bullet}, d\right\rangle$, where the differential $d$ is the alternating sum of the face maps.

Proof. For any $i \geq 0$, we have

$$
E_{i}=E \otimes_{\Delta} k_{i},
$$

where $k_{i} \in \operatorname{Fun}(\Delta, k)$ is the functor represented by $[i+1] \in \Delta$, the set with $(i+1)$ elements. The differential $d: E_{\bullet}+1 \rightarrow E_{\bullet}$ is induced by a differential $d: k_{\bullet+1} \rightarrow k_{i}$. To prove the claim, it suffices to prove that the complex $\left\langle k_{\bullet}, d\right\rangle$ is a resolution of the constant functor $k \in \operatorname{Fun}(\Delta, k)$. This we may prove pointwise, evaluating at all objects $[n] \in \Delta$. In other words, it suffices to prove the claim for all representable $E . \in \operatorname{Fun}\left(\Delta^{o}, k\right)$. This amounts to the trivial computation of chain homology of the standard simplices. 
In this paper, we will also need a slightly refined version of Lemma 1.1. Namely, it is well-known that, given a simplicial vector space $E_{\text {. }}$, instead of the complex $\left\langle E_{\bullet}, d\right\rangle$ one can consider the normalized quotient complex $\left\langle N\left(E_{\bullet}\right), d\right\rangle$, where for any $n, N\left(E_{n}\right)$ is the quotient of $E_{n}$ by the images of all the degeneration maps. Then the quotient map $E_{\bullet} \rightarrow N\left(E_{\bullet}\right)$ is a quasiisomorphism. Moreover, it has been proved a long time ago by A. Dold [Do], and independently by D. Kan, that the correspondence $E_{\bullet} \mapsto\left\langle N\left(E_{\bullet}\right), d\right\rangle$ is actually an equivalence of abelian categories between $\operatorname{Fun}\left(\Delta^{o}, k\right)$ and the category $C^{\leq 0}(k)$ of complexes of $k$-vector spaces which are trivial in positive (cohomological) degrees. Moreover, one can replace the category of $k$-vector spaces with an arbitrary abelian category $\mathcal{C}$ - we still have an equivalence

$$
\mathrm{D}: \operatorname{Fun}\left(\Delta^{o}, \mathcal{C}\right) \stackrel{\sim}{\longrightarrow} C^{\leq 0}(\mathcal{C})
$$

between simplicial objects in $\mathcal{C}$ and complexes in $\mathcal{C}$ which are trivial in positive degree. This is now known as the Dold-Kan equivalence. As in Lemma 1.1, the complex corresponding to an object $E . \in \operatorname{Fun}\left(\Delta^{o}, \mathcal{C}\right)$ represents the object $L^{\cdot} \tau_{!}\left(E_{\bullet}\right) \in \mathcal{D}^{-}(\mathcal{C})$, where, as before, the functor $\tau_{!}: \operatorname{Fun}\left(\Delta^{o}, \mathcal{C}\right) \rightarrow \mathcal{C}$ is induced by the projection to the point $\tau: \Delta^{o} \rightarrow$ pt.

We note that the opposite $\mathcal{C}^{o}$ to an abelian category $\mathcal{C}$ is also abelian. Since we obviously have $\operatorname{Fun}\left(\Delta^{o}, \mathcal{C}^{o}\right) \cong \operatorname{Fun}(\Delta, \mathcal{C})^{o}$, the Dold-Kan equivalence has a version for cosimplicial objects - we have

$$
\operatorname{Fun}\left(\Delta^{o}, \mathcal{C}\right) \cong C^{\geq 0}(\mathcal{C}),
$$

where $C^{\geq 0}(\mathcal{C})$ is the category of complexes in $\mathcal{C}$ which are trivial in negative degrees. The complex corresponding to an object $E$. $\in \operatorname{Fun}(\Delta, \mathcal{C})$ represents the object $R^{\bullet} \tau_{*}\left(E_{\text {. }}\right) \in \mathcal{D}^{-}(\mathcal{C})$

In the case $\mathcal{C}=k$-Vect, both $\operatorname{Fun}\left(\Delta^{o}, k\right)$ and $C^{\leq 0}(k)$ are symmetric tensor categories. The Dold-Kan equivalence is compatible with the tensor structure to some extent - there exists a functorial map

$$
\mathrm{D}\left(V_{\bullet}\right) \otimes \mathrm{D}\left(W_{\bullet}\right) \rightarrow \mathrm{D}\left(V_{\bullet} \otimes W_{\bullet}\right)
$$

for any $V_{\bullet}, W_{.} \in \operatorname{Fun}\left(\Delta^{o}, k\right)$, and this map is compatible with the associativity and commutativity morphisms. It is also a quasiisomorphism (which cannot be inverted - this is the well-known "commutative cochain problem"). In addition, if the complexes $\mathrm{D}\left(V_{\bullet}\right), \mathrm{D}\left(W_{\bullet}\right)$ are concentrated in degrees respectiely between 0 
and $n$ and between 0 and $m$, then $\mathrm{D}\left(V_{\bullet} \otimes W_{\bullet}\right)$ is concentrated in degrees between 0 and $m+n$.

We will need two refinements of the Dold-Kan equivalence. One is a simplicial characterization of acyclic complexes. Let $\Delta_{+} \subset \Delta$ be the category whose objects are all objects of $\Delta$, and whose morphisms are those maps between finite linearly ordered sets which preserve the first element. Then the Dold-Kan equivalence induces an equivalence between the category $\operatorname{Fun}\left(\Delta_{+}^{o}, k\right)$ and the category of $k$-vector spaces $V$. graded by non-positive integers. The restriction functor $\operatorname{Fun}\left(\Delta^{o}, k\right) \rightarrow \operatorname{Fun}\left(\Delta_{+}^{o}, k\right)$ corresponds under this equivalence to forgetting the differential in the complex. The embedding $\Delta_{+} \subset \Delta$ admits an obvious adjoint $s: \Delta \rightarrow \Delta_{+}$(adding the first element). The restriction $s^{*}: \operatorname{Fun}\left(\Delta_{+}^{o}, k\right) \rightarrow$ $\operatorname{Fun}\left(\Delta^{o}, k\right)$ is adjoint to the restriction $\operatorname{Fun}\left(\Delta^{o}, k\right) \rightarrow \operatorname{Fun}\left(\Delta_{+}^{o}, k\right)$. This immediately implies that under the Dold-Kan equivalence, $s^{*}$ becomes the functor

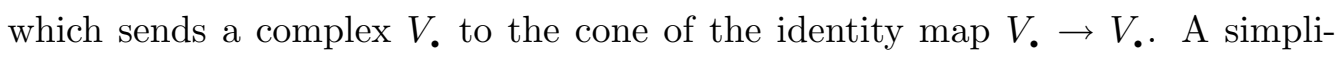
cial vector space $E$. is said to be homotopic to 0 if it is of the form $s^{*} E^{\prime}$ for some $E^{\prime} \in \operatorname{Fun}\left(\Delta_{+}^{o}, k\right)$; we see that a complex becomes homotopic to 0 under the Dold-Kan equivalence if and only if it is acyclic. One can also show that fixing $E^{\prime} \in \operatorname{Fun}\left(\Delta_{+}^{o}, k\right)$ such that $s^{*} E^{\prime} \cong E$. is equivalent to fixing a contracting chain homotopy for the complex corresponding to $E_{\text {. }}$.

Another refinement concerns filtered complexes. Recall that for any complex $E_{.} \in C_{\leq 0}(\mathcal{C})$ in an abelian category $\mathcal{C}$, its stupid filtration is the increasing filtration $F . E$. given by

$$
F_{m} E_{n}= \begin{cases}E_{n}, & n<m, \\ 0, & n \geq m .\end{cases}
$$

In terms of the Dold-Kan equivalence, the stupid filtration is given by

$$
F_{m} \mathrm{D}\left(E_{\bullet}\right)=\mathrm{D}\left(j_{m !} j_{m}^{*} E_{\bullet}\right),
$$

where $j_{m}: \Delta_{\leq m}^{o} \rightarrow \Delta^{o}$ is the embedding of the full subcategory $D_{\leq m}^{o} \subset \Delta^{o}$ spanned by objects $[1], \ldots,[m] \in \Delta^{o}$ (in fact, the Dold-Kan equivalence $\mathrm{D}$ induces an equivalence between $\operatorname{Fun}\left(\Delta_{\leq m}^{o}, \mathcal{C}\right)$ and the category of complexes concentrated in homological degrees $0, \ldots, m-1)$. Taking the complex $E . \in C^{\leq 0}(\mathcal{C})$ with its stupid filtration defines a functor

$$
C^{\leq 0}(\mathcal{C}) \rightarrow \mathcal{D F}(\mathcal{C})
$$


into the so-called filtered derived category $\mathcal{D F}(\mathcal{C})$ of the category $\mathcal{C}$. Formally, $\mathcal{D F}(\mathcal{C})$ is obtained by inverting the filtered quasiisomorphisms in the category of complexes $E$. in $\mathcal{C}$ equipped with an exhaustive increasing filtration $F$. $E$. whose terms are numbered by non-negative integers (this is a version of the construction in $[\mathrm{BBD}$, Section 3.1]). In practice, one can equally well define $\mathcal{D F}(\mathcal{C})$ as the derived category of the functor category $\operatorname{Fun}(\mathbb{N}, \mathcal{C})$, where $\mathbb{N}$ is the set of nonnegative integers with it natural order, consider as a small category in the usual way. The functor (1.7) obviously extends to the derived category $\mathcal{D}(\mathcal{C} \leq 0(\mathcal{C})$ ); composing this with the Dold-Kan equvalence, we obtain a triangulated functor

$$
\text { Real }: \mathcal{D}\left(\operatorname{Fun}\left(\Delta^{o}, \mathcal{C}\right)\right) \rightarrow \mathcal{D F}(\mathcal{C}) \cong \mathcal{D}(\operatorname{Fun}(\mathbb{N}, \mathcal{C})) .
$$

This functor is itself an equivalence, although we will not need this (see [BBD, Section 3.1] and [BGS]).

1.3. Effectively finite simplicial objects. We will now make a digression and introduce a certain finiteness condition on filtered and simplicial objects. For any object $\left\langle E_{\bullet}, F_{\bullet}\right\rangle \in \mathcal{D F}(\mathcal{C})$ and any integer $m \geq 0$, denote by $F^{m} E_{\bullet} \in \mathcal{D}(\mathcal{C})$ the cone of the natural map $F_{m} E$. $\rightarrow E_{\text {. }}$

Definition 1.2. An object $\left\langle E_{\bullet}, F_{.}\right\rangle \in \mathcal{D F}(\mathcal{C})$ is said to be effectively finite if for any $m \geq 0$, there exists $m^{\prime}>m$ such that the natural map

$$
F^{m} E . \rightarrow F^{m^{\prime}} E \text {. }
$$

of objects in $\mathcal{D}(\mathcal{C})$ is equal to 0 . An object $E . \in \mathcal{D}\left(\operatorname{Fun}\left(\Delta^{o}, \mathcal{C}\right)\right)$ is said to be effectively finite if so is $\operatorname{Real}\left(E_{\bullet}\right) \in \mathcal{D F}(\mathcal{C})$.

Lemma 1.3. (i) The cone $E_{\text {. }}^{\prime \prime}$ of a map $E_{\text {. }} \rightarrow E_{\text {. }}^{\prime}$ between effectively finite $E_{.}, E_{.}^{\prime} \in \mathcal{D F}(\mathcal{C})$ is itself effectively finite.

(ii) Assume that $\left\langle E_{.}, F_{\bullet}\right\rangle,\left\langle E_{\bullet}, F_{.}^{\prime}\right\rangle \in \mathcal{D F}(\mathcal{C})$ give two filtrations on the same object $E . \in \mathcal{D}(\mathcal{C})$ which are cofinal in the sense that for every $m \geq 0$ there exists $m^{\prime} \geq m$ such that the map $F_{m} E$. $\rightarrow$ E. factors through

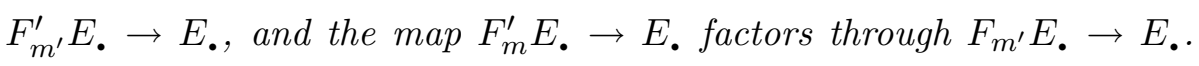
Then $\left\langle E_{.}, F_{.}\right\rangle$is effectively finite if and only if so is $\left\langle E_{\bullet}, F_{.}^{\prime}\right\rangle$.

Proof. The second claim is obvious. To prove the first, fix an integer $m$, and choose $m_{0}^{\prime}>m$ so that the map $F^{m} E$. $\rightarrow F^{m_{0}^{\prime}} E$. is equal to 0 . Then the 


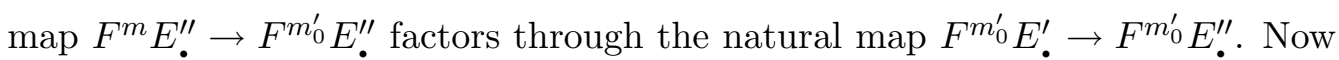
choose $m^{\prime}>m_{0}^{\prime}$ so that $F^{m_{0}^{\prime}} E^{\prime} \rightarrow F^{m^{\prime}} E^{\prime}$. is also equal to 0 .

We will need another characterization of effectively finite filtered and simplicial objects. Let $\mathcal{D} \mathrm{F}_{[0,1]}(\mathcal{C}) \subset \mathcal{D F}(\mathcal{C})$ be the full subcategory of objects $\left\langle E_{.}, F_{\text {. }}\right\rangle$ with two-step filtration: $F_{1} E_{.}=E_{.}$. For every $m \geq 0$ and a filtration $F$. on an object $E_{\bullet}$, define a new filtration $\tau_{m}(F)$. by $\tau_{m}(F)_{0} E_{\bullet}=F_{m} E_{\bullet}, \tau_{m}(F)_{1} E_{\bullet}=E_{\text {. }}$. Setting $\left\langle E_{\bullet}, F_{\bullet}\right\rangle \mapsto\left\langle E_{\bullet}, \tau_{m}(F).\right\rangle$ gives a functor

$$
\tau_{m}: \mathcal{D F}(\mathcal{C}) \rightarrow \mathcal{D F}_{[0,1]}(\mathcal{C}) .
$$

(An alternative description: $\tau_{m}=L^{\bullet} \chi_{!}^{m}$, where $\chi^{m}: \mathbb{N} \rightarrow\{0,1\}$ is the characteristic function of the segment $[m+1, \infty[\subset \mathbb{N}$.) We also have two tautological functors $\iota, \iota^{\prime}: \mathcal{D}(\mathcal{C}) \rightarrow \mathcal{D F}_{[0,1]}(\mathcal{C})$ given by $F_{0} \iota\left(E_{\bullet}\right)=0, F_{0} \iota^{\prime}\left(E_{\bullet}\right)=E_{\text {. }}$. It is a standard fact that for any object $E, E^{\prime} \in \mathcal{D}(\mathcal{C})$, we have $\mathrm{RHom}^{\bullet}\left(\iota^{\prime}(E), \iota\left(E^{\prime}\right)\right)=0$.

Lemma 1.4. An object $E . \in \mathcal{D F}(\mathcal{C})$ is effectively finite if and only if for any $m \geq 0$ there exists an integer $m^{\prime}>m$ such that the natural map $q: \tau_{m}\left(F_{m^{\prime}} E_{\bullet}\right) \rightarrow$ $\tau_{m}\left(E_{\bullet}\right)$ admits a right-inverse $p: \tau_{m}\left(E_{\bullet}\right) \rightarrow \tau_{m}\left(F_{m^{\prime}} E_{\bullet}\right)$ (that is, $q \circ p=$ id as an endomorphism of $\tau_{m}\left(E_{\text {. }}\right)$ ).

Proof. The cone of the map $q$ is naturally identified with $\iota\left(F^{m^{\prime}} E_{\text {. }}\right)$, so that the obstruction to the existence of a splitting map $p$ is the composition map

$$
\tau_{m}\left(E_{\text {. }}\right) \rightarrow \iota\left(F^{m} E_{\bullet}\right) \rightarrow \iota\left(F^{m^{\prime}} E_{\text {. }}\right) ;
$$

but the cone of the map in the left-hand side is $\iota^{\prime}\left(F_{m} E_{\text {. }}\right)[1]$, and this is orthogonal to $\iota\left(F^{m^{\prime}} E_{\text {. }}\right)$.

Corollary 1.5. Assume given an associative algebra $B$ over $k$ and an effectively finite object $E . \in \mathcal{D}\left(\operatorname{Fun}\left(\Delta^{o}, B \bmod \right)\right)$ in the derived category of simplicial $B$ modules.

(i) For any associative $k$-algebras $B, B^{\prime}$ and any effectively finite objects $E . \in \mathcal{D}\left(\operatorname{Fun}\left(\Delta^{o}, B \bmod \right)\right), E^{\prime} \in \mathcal{D}\left(\operatorname{Fun}\left(\Delta^{o}, B^{\prime} \bmod \right)\right)$, the product $E_{.} \otimes E_{.}^{\prime} \in \mathcal{D}\left(\operatorname{Fun}\left(\Delta^{o},\left(B \otimes B^{\prime}\right) \bmod \right)\right)$ is effectively finite.

(ii) For any integer $n \geq 1$, associative $k$-algebra $B$, and effectively finite object $E . \in \mathcal{D}\left(\operatorname{Fun}\left(\Delta^{o}, B \bmod \right)\right)$, the $n$-th tensor power

$$
E^{\otimes n} \in \mathcal{D}\left(\operatorname{Fun}\left(\Delta^{o}, B^{\otimes n} \bmod \underset{\mathbb{Z} / n \mathbb{Z}}{ }\right)\right)
$$


is effectively finite in the derived category of simplicial $\mathbb{Z} / n \mathbb{Z}$-equivariant $B^{\otimes n}$-modules.

Proof. For the first claim, use the criterion of Lemma 1.4: choose $m^{\prime}$ large enough so that for both $E$. and $E^{\prime}$. there exist the splitting maps $p, p^{\prime}$, and take $p \otimes p^{\prime}$ as the required splitting map for $E$. $\otimes E^{\prime}$. For the second claim, we note that for any $m$, we obviously have

$$
F_{m} E_{\bullet}^{\otimes n} \subset\left(F_{m} E_{\bullet}\right)^{\otimes n}, \quad\left(F_{m} E_{\bullet}\right)^{\otimes n} \subset F_{m n} E_{\bullet}^{\otimes n},
$$

so that the stupid filtration on $E^{\otimes n}$ is cofinal with the $n$-th power of the stupid filtration on $E$. Since the Dold-Kan equivalence is compatible, up to a quasiisomorphism, with the tensor product, this means that we can apply Lemma 1.3 (ii) and replace $\operatorname{Real}\left(E^{\otimes n}\right)$ with $\operatorname{Real}\left(E_{\bullet}\right)^{\otimes n}$ (the tensor product is taken with respect to the pointwise tensor structure on $\mathcal{D F}(\mathcal{C}) \cong \mathcal{D}(\operatorname{Fun}(\mathbb{N}, \mathcal{C})))$. Now again use Lemma 1.4: the functor $\tau_{m}$ obviously respects the tensor structure, and the required splitting map is given by $p^{\otimes n}$, where $p$ is the corresponding splitting map for the effectively finite $E$.

Finally, we remark that by duality, as in Subsection 1.2, all the material in this subsection immediately extends to cosimplicial objects; we leave it to the reader.

1.4. Fibrations and cofibrations. We now return to small categories. One important special class of functors $f: \Gamma^{\prime} \rightarrow \Gamma$ between small categories is that of fibrations introduced by Grothendieck in [G, Exposé VI]. Namely, for any functor $\sigma: \Gamma^{\prime} \rightarrow \Gamma$ between small categories $\Gamma^{\prime}$ and $\Gamma$, and for and any object $[a] \in \Gamma$, the fiber $\Gamma_{[a]}^{\prime}$ of the functor $\sigma$ over the object $[a]$ is by definition the subcategory in $\Gamma^{\prime}$ of all objects $\left[a^{\prime}\right] \in \Gamma^{\prime}$ such that $\sigma\left(\left[a^{\prime}\right]\right)=[a]$ and all morphisms $f$ such that $\sigma(f)=\mathrm{id}_{[a]}$.

Definition 1.6. Assume given a functor $\sigma: \Gamma^{\prime} \rightarrow \Gamma$. A morphism $f:[a] \rightarrow[b]$ in $\Gamma$ is called Cartesian if it has the following universal property:

any morphism $f^{\prime}:\left[a^{\prime}\right] \rightarrow[b]$ in $\Gamma^{\prime}$ such that $\sigma\left(f^{\prime}\right)=\sigma(f)$ factors through $f$ by means of a unique map $\left[a^{\prime}\right] \rightarrow[a]$ in the fiber $\Gamma_{\sigma([a])}^{\prime}$.

The functor $\sigma$ is a fibration if 
(i) for any map $f:[a] \rightarrow[b]$ in $\Gamma$ and any object $\left[b^{\prime}\right] \in \Gamma_{[b]}^{\prime}$, there exists an object $f^{*}\left[b^{\prime}\right] \in \Gamma_{[a]}^{\prime}$ and a Cartesian map $f^{\prime}: f^{*}\left[b^{\prime}\right] \rightarrow\left[b^{\prime}\right]$ such that $\sigma\left(f^{\prime}\right)=f$, and

(ii) the composition of two Cartesian maps is Cartesian.

The functor $\sigma$ is a cofibration if the oppositie functor $\sigma^{o}: \Gamma^{\prime} o \rightarrow \Gamma^{o}$ is a fibration.

By the universal property of Cartesian morphisms, the correspondence $\left[b^{\prime}\right] \mapsto$ $f^{*}\left[b^{\prime}\right]$ in (i) is functorial with respect to $\left[b^{\prime}\right] \in \Gamma_{[b]}^{\prime}$, so that we have a functor $f^{*}: \Gamma_{[b]}^{\prime} \rightarrow \Gamma_{[a]}^{\prime}$. In addition, for any pair $f, g$ of composable maps in $\Gamma$, we have a map $g^{*} \circ f^{*} \rightarrow(f \circ g)^{*}$. The condition (ii) insures that this map is an isomorphism. Thus given a fibration $\sigma: \Gamma^{\prime} \rightarrow \Gamma$, one can consider the correspondence $[a] \mapsto \Gamma_{[a]}^{\prime}$, $f \mapsto f^{*}$ as a contravariant weak functor from $\Gamma$ to the category of small categories ("weak" here means that $f^{*} g^{*}$ is only canonically isomorphic, not equal to $(g f)^{*}$ ). Conversely, every such weak functor defines a fibration $\sigma: \Gamma^{\prime} \rightarrow \Gamma$ (objects of $\Gamma^{\prime}$ are pairs $\left.\left\langle[a],\left[a^{\prime}\right]\right\rangle,[a] \in \Gamma,\left[a^{\prime}\right] \in \Gamma_{[a]}^{\prime}\right]$. For cofibrations, the picture is similar, but the weak functor is covariant: for any map $f:[a] \rightarrow[b]$, we have a natural functor $f_{!}: \Gamma_{[a]}^{\prime} \rightarrow \Gamma_{[b]}^{\prime}$. This is known nowadays as the Grothendieck construction. We refer the reader to $[\mathrm{G}$, Exposé VI] for a precise definition of a weak functor ("pseudo-foncteur") and further details.

We note that the condition (i) of Definition 1.6 is analogous to the covering homotopy property in algebraic topology, so that in a sense, fibrations of small categories are analogous to fibrations in the topological sense. For cofibrations, the topological analogy is still a fibration. Thus the term "cofibration" is somewhat misleading, and nowadays cofibrations in the sense of Definition 1.6 are sometimes called op-fibrations. For better or for worse, we will stick to the original terminology of Grothendieck. However, the topological analogy is useful, because categorical fibrations and cofibrations do indeed enjoy nice properties similar to those of fibrations in algebraic topology.

Lemma 1.7. Let $\sigma: \Gamma^{\prime} \rightarrow \Gamma$ be a cofibration.

(i) For any object $E \in \operatorname{Fun}(\Gamma, k)$, we have a natural isomorphism

$$
L^{\cdot} \sigma_{!} \sigma^{*} E \cong E \otimes L^{\cdot} \sigma_{!} \sigma^{*} k
$$

and this isomorphism is functorial in $E$. 
(ii) For any small category $\Gamma_{1}$ and a functor $\rho: \Gamma_{1} \rightarrow \Gamma$, the fibered product $\sigma^{\prime}: \Gamma_{1}^{\prime}=\Gamma^{\prime} \times_{\Gamma} \Gamma_{1} \rightarrow \Gamma_{1}$ is also a cofibration, and we have a natural base change isomorphism

$$
L^{\bullet} \sigma_{!}^{\prime} \circ \rho^{*} \cong \rho^{*} \circ L^{\bullet} \sigma_{!}
$$

associated to the Cartesian diagram

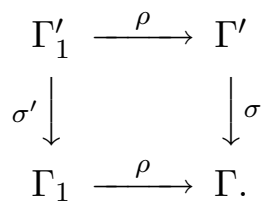

Proof. Both facts are pretty standard, but we include a proof for the convenience of the reader. We note that the fibered product of small categories is understood in the most straightforward sense - the set of objects is the fibered product of sets of objects, the set of morphisms is the fibered product of sets of morphisms. The map in (1.9) is obtained by adjunction from the natural map

$$
\rho^{*} \rightarrow \rho^{*} \circ \sigma^{*} \circ L^{*} \sigma_{!} \cong \sigma^{*} \circ \rho^{*} \circ L^{*} \sigma_{!} .
$$

To check that $\sigma^{\prime}: \Gamma_{1}^{\prime} \rightarrow \Gamma_{1}$ is indeed a fibration is an elementary exercize left to the reader. Moreover, for any object $[a] \in \Gamma_{1}$, we have a natural isomorphism $\rho^{\prime}: \sigma_{[a]}^{\prime} \cong \sigma_{\rho([a])}$. Thus to prove that the already defined map in (1.9) is an isomorphism, it suffices to prove that for any $E \in \operatorname{Fun}\left(\Gamma^{\prime}, k\right)$ and any $[a] \in \Gamma$, we have

$$
L^{\bullet} \sigma_{!} E([a]) \cong H_{\cdot}\left(\sigma_{[a]},\left.E\right|_{\sigma_{[a]}}\right)
$$

In other words, we may assume that $\Gamma_{1}=\mathrm{pt}$, and $\rho=\iota_{[a]}:$ pt $\rightarrow \Gamma$ is the embedding of an object $[a] \in \Gamma$. Moreover, since $\operatorname{Fun}\left(\Gamma^{\prime}, k\right)$ is generated by representable functors, it suffices to prove (1.10) when $E=\iota_{[b] !} k$ for some $[b] \in \Gamma^{\prime}$. Then the left-hand side is tautologically isomorphic to $k[\Gamma([b], \sigma([a]))]$, while since $\sigma$ is a cofibration, the restriction $\left.E\right|_{\sigma_{[a]}}$ in the right-hand side splits as

$$
\bigoplus_{f \in \Gamma(\sigma([b]),[a])} \iota_{\left(f_{:}[b]\right) !} k
$$

and we tautologically have $H^{\bullet}\left(\sigma_{[a]}, \iota_{\left(f_{!}[b]\right) !} k\right) \cong k$. This proves (1.10) and (i). To prove (i), we note that (1.8) is also an immediate corollary of (1.10), since we obviously have

$$
H_{.}\left(\Phi, V^{\Phi}\right) \cong V \otimes H_{\bullet}(\Phi, k)
$$


for any small category $\Phi$ and the constant functor $V^{\Phi} \in \operatorname{Fun}(\Phi, k)$ which sends each object $[a] \in \Phi$ to a fixed $k$-vector space $V$.

There is also a dual statement for fibrations which we leave to the reader. The only difference is that since tensor products only commute with infinite sums, not with infinite products, (1.11) only holds for cohomology under additional assumptions - for instance, it suffices to require that $V$ is finite-dimensional, or that $\Gamma$ is finite. Therefore the projection formula (1.8) also requires some assumptions. For example, if all fibers of a projection $\sigma: \Gamma^{\prime} \rightarrow \Gamma$ are finite, then we do we have a canonical isomorphism

$$
R^{\bullet} \sigma_{*} \sigma^{*} E \cong E \otimes R^{\bullet} \sigma_{*} k
$$

for any $E \in \operatorname{Fun}(\Gamma, k)$.

A cofibration $\sigma: \Gamma^{\prime} \rightarrow \Gamma$ is called discrete if all its fibers $\Gamma_{[a]}^{\prime}$ are discrete categories. The Grothendieck construction identifies discrete fibrations over $\Gamma$ with covariant functors from $\Gamma$ to the category of sets (since the fibers are discrete, these are not "weak" functors but functors in the usual sense). Since discrete categories obviously have no higher cohomology, the direct image functor $\sigma_{\text {! }}$ associated to a discrete fibration $\sigma: \Gamma^{\prime} \rightarrow \Gamma$ is exact. Analogously, a fibration is called discrete if all its fibers are discrete; if $\sigma: \Gamma^{\prime} \rightarrow \Gamma$ is a discrete fibration with finite fibers, then the functor $\sigma_{*}$ is exact.

A functor $\sigma: \Gamma^{\prime} \rightarrow \Gamma$ which is both a fibration and a cofibration is called a bifibration. It immediately follows from the definitions that a fibration $\sigma: \Gamma^{\prime} \rightarrow \Gamma$ is a bifibration if and only if for any objects $[a],[b] \in \Gamma$ and a morphism $f:[a] \rightarrow$ $[b]$, the functor $f^{*}: \sigma_{[b]} \rightarrow \sigma_{[a]}$ admits an adjoint functor $f_{!}: \sigma_{[a]} \rightarrow \sigma_{[b]}$ (for details, see [G, Exposé VI]). In particular, if all the fibers $\sigma_{[a]}$ of a bifibration $\sigma$ are groupoids, all the functors $f^{*}$ must be equivalences. Thus a discrete bifibration $\Gamma^{\prime} \rightarrow \Gamma$ over a connected $\Gamma$ must be a product $\Gamma^{\prime}=\Gamma \times S$ of $\Gamma$ and a discrete category $S$. However, if the fibers are non-trivial groupoids, the bifibration may be non-trivial. We will in fact need one such (the projection $\pi$ in (1.12)). We will also need one general property of bifibrations whose fibers are groupoids with finite automorphism groups. Namely, recall that for any finite group $G$ and any $G$-module $V$, we have a natural trace map $\operatorname{tr}: H_{0}(G, V) \rightarrow H^{0}(G, V)$ given by averaging over the group. This immediately generalizes to groupoids, and further, we introduce the following. 
Definition 1.8. For any bifibration $\sigma: \Gamma^{\prime} \rightarrow \Gamma$ whose fibers are groupoids with finite automorphism groups, the trace map $\operatorname{tr}: \sigma_{!} \rightarrow \sigma_{*}$ is given by

$$
\operatorname{tr}: \sigma_{!} F([a])=H_{0}\left(\sigma_{[a]},\left.F\right|_{\sigma_{[a]}}\right) \rightarrow H^{0}\left(\sigma_{[a]},\left.F\right|_{\sigma_{[a]}}\right)=\sigma^{*} F([a])
$$

for any $F \in \operatorname{Fun}\left(\Gamma^{\prime}, k\right),[a] \in \Gamma$.

We leave it to the reader to check that this indeed gives a well-defined map of functors $\sigma_{!} \rightarrow \sigma_{*}$.

1.5. Versions of the cyclic category. The small category that we need for cyclic homology is A. Connes' cyclic category $\Lambda$. This is a small category whose objects $[n]$ are indexed by positive integers $n$. Maps between $[n]$ and $[m]$ can be defined in various equivalent ways; for the convenience of the reader, we recall two of these descriptions.

Topological description. The object $[n]$ is thought of as a "wheel" - the circle $S^{1}$ with $n$ distinct marked points. A continuous map $f:[n] \rightarrow[m]$ is good if it sends marked points to marked points, has degree 1 , and is non-descreasing with respect to the orientation of the circle: for any interval $[a, b] \subset S^{1}$, its image $f([a, b]) \subset S^{1}$ is the interval $[f(a), f(b)]$ with the same orientation as $[a, b]$. Morphisms from $[n]$ to $[m]$ in the category $\Lambda$ are homotopy classes of good maps $f:[n] \rightarrow[m]$.

Combinatorial description. Consider the category Cycl of linearly ordered sets equipped with an order-preserving endomorphism $\sigma$. Let $[n] \in \mathrm{Cycl}$ be the set $\mathbb{Z}$ with the natural linear order and endomorphism $\sigma: \mathbb{Z} \rightarrow \mathbb{Z}, \sigma(a)=a+n$. Let $\Lambda_{\infty} \subset$ Cycl be the full subcategory spanned by $[n], n \geq 1$. For any $[n],[m] \in \Lambda_{\infty}$, the set $\Lambda_{\infty}([n],[m])$ is acted upon by the endomorphism $\sigma$ (on the left, or on the right, by definition it does not matter). We define the set of maps $\Lambda([n],[m])$ in the category $\Lambda$ by $\Lambda([n],[m])=\Lambda_{\infty}([n],[m]) / \sigma$.

We refer the reader for further details to [L, Chapter 6] (in particular, there is an explicit description of maps in $\Lambda$ by generators and relations).

For our applications, we will need a version of the category $\Lambda$ which we will call $p$-cyclic category and denote by $\Lambda_{p}$ - here $p$ is an integer $\geq 1, \Lambda_{1}$ is the original $\Lambda$. The objects $[n] \in \Lambda_{p}$ also correspond to positive integers; however, in the 
combinatorial description above, we let the set of maps to be

$$
\Lambda_{p}([n],[m])=\Lambda_{\infty}([n],[m]) / \sigma^{p} .
$$

In a sense, the category $\Lambda_{p}$ is a $p$-fold cover of the category $\Lambda$. More precisely, we have a natural projection functor $\pi: \Lambda_{p} \rightarrow \Lambda$ which is identical on objects, and corresponds to taking the quotient by $\sigma$ on the morphisms sets. It is immediate to check that $\pi: \Lambda_{p} \rightarrow \Lambda$ is a bifibration; all its fibers are isomorphic to $\mathrm{pt}_{p}$, the groupoid with one object whose automorphism group is $\mathbb{Z} / p \mathbb{Z}$. We also have an obvious natural embedding $i: \Lambda_{p} \rightarrow \Lambda,[n] \mapsto[n p]$. To sum up, we have a diagram

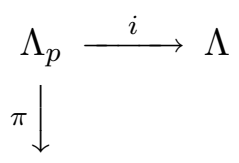

$\Lambda$

of small categories and functors between them, such that the left column is a bifibration with fiber $\mathrm{pt}_{p}$.

The simplicial category $\Delta^{o}$ - that is, the opposite to the category $\Delta$ of finite linearly ordered sets - is naturally embedded into $\Lambda$. In fact, it is even embedded in the category $\Lambda_{\infty}$ used in in the combinatorial description above. Namely, $\Delta^{o} \subset \Lambda_{\infty}$ contains all its objects $[n], n \geq 1$, and those morphisms $\mathbb{Z} \rightarrow \mathbb{Z}$ between them which preserve $0 \in \mathbb{Z}$. This descends to an embedding $\Delta^{o} \subset \Lambda_{p}$ for any $p \geq 1$. We denote these embeddings by $j: \Delta^{o} \rightarrow \Lambda, j_{p}: \Delta^{o} \rightarrow \Lambda_{p}, j_{\infty}: \Delta^{o} \rightarrow \Lambda_{\infty}$. The embedding $j_{p}$ obviously extends to an embedding $\bar{j}_{p}: \Delta^{o} \times \mathrm{pt}_{p} \rightarrow \Lambda_{p}$. The category $\Lambda$ is self-dual - we have $\Lambda^{o} \cong \Lambda$. The same is true for the categories $\Lambda_{\infty}$ and $\Lambda_{p}$ for higher $p$. By duality, we obtain embeddings $j^{o}: \Delta \rightarrow \Lambda, j_{p}^{o}: \Delta \rightarrow \Lambda_{p}$.

The embedding $j: \Delta^{o} \rightarrow \Lambda$ obviously cannot be a cofibration, but it is equivalent to one - it can be factored as

$$
\Delta^{o} \longrightarrow \widetilde{\Delta}^{o} \stackrel{\widetilde{j}}{\longrightarrow} \Lambda,
$$

for some small category $\widetilde{\Delta}^{o}$ so that the first map is an equivalence, and the second one is a cofibration. The cofibration $\widetilde{j}$ is in fact discrete, and it corresponds by Grothendieck construction to the functor $\Lambda \rightarrow$ Sets which sends a wheel $[n]$ to the set of its marked points. Equivalently, this is the functor represeted by $[1] \in \Lambda$, so that $\widetilde{\Delta}^{o}$ is the category of objects $[n] \subset \Lambda$ equipped with a map $[1] \rightarrow[n]$. In the topological description above, $\widetilde{\Delta}^{o}$ is the category of wheels with one distinguished 
point. The same construction works for $j_{p}: \Delta^{o} \rightarrow \Lambda$ - if we denote by $\widetilde{\Delta}_{p}^{o}$ the category of objects $[n] \in \Lambda_{p}$ equipped with a map $[1] \rightarrow[n]$, then the forgetful functor $\widetilde{\Delta}_{p}^{o}$ is a discrete cofibration, and $j_{p}$ factors through this cofibration by an equivalence $\Delta^{o} \rightarrow \widetilde{\Delta}_{p}^{o}$. We leave it to the reader to check that we have a natural commutative diagram

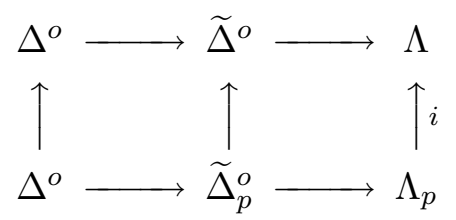

with Cartesian square on the right-hand side, where $i: \Lambda_{p} \rightarrow \Lambda$ is the embedding from (1.12).

Since equivalences of small categories induce equivalences of functor categories, all this means that the embeddings $j: \Delta^{o} \rightarrow \Lambda, j_{p}: \Delta^{o} \rightarrow \Lambda_{p}$ have all the cohomological properties of discrete cofibrations: the functors $j_{!}, j_{p}$ ! are exact, and we have the projection formula and the base change, as in Lemma 1.7. By duality, the embeddings $j^{o}: \Delta \rightarrow \Lambda, j_{p}^{o}: \Delta \rightarrow \Lambda_{p}$ are equivalent to discrete fibrations with finite fibers (with all the cohomological properties that this entails).

As far as the map $\pi$ from (1.12) is concerned, we have a cartesian square

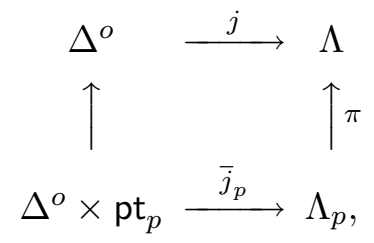

where on the left-hand side we have the natural projection onto the first summand.

1.6. Cyclic homology and periodicity. Objects in $\operatorname{Fun}(\Lambda, k)$ are commonly called cyclic $k$-vector spaces; we will generalize this and call objects in $\operatorname{Fun}\left(\Lambda_{p}, k\right)$ p-cyclic k-vector spaces.

Definition 1.9. Assume given a $p$-cyclic vector space $E$. Then its cyclic homology $H C$. $(E)$ is defined by

$$
H C .(E)=H_{\bullet}\left(\Lambda_{p}, E\right),
$$

and its Hochschild homology HH. $(E)$ is defined by

$$
H_{\bullet}(E)=H_{\bullet}\left(\Delta^{o}, j_{p}^{*} E\right) .
$$


Hochschild homology is easy to compute by an explicit complex, as in Lemma 1.1. It was discovered by A. Connes that cyclic and Hochschild homology are related. Namely, for any cyclic $k$-vector space $E \in \operatorname{Fun}(\Lambda, k)$, there exists a canonical periodicity map $u: H C .(E) \rightarrow H C_{\bullet_{-} 2}(E)$ and a canonical long exact sequence

$$
H H_{.}(E) \longrightarrow H_{.}(E) \stackrel{u}{\longrightarrow} H_{\cdot-2}(E) \longrightarrow
$$

known as the Connes' exact sequence. It is usually constructed on the level of explicit complexes, as in e.g. [L, Chapter 2]. We will need to reformulate it in a slightly more abstract way (which will also yield generalization to $p$-cyclic vector spaces).

Namely, by adjunction, we have natural maps $k \rightarrow j_{*}^{o} k, j ! k \rightarrow k$. It turns out that there exists a connecting map $B: j_{*}^{o} k \rightarrow j_{!} k$ such that the sequence

$$
0 \longrightarrow k \longrightarrow j_{*}^{o} k \stackrel{B}{\longrightarrow} j_{!} k \longrightarrow k \longrightarrow 0
$$

in the category $\operatorname{Fun}(\Lambda, k)$ is exact. Explicitly, for any $[n] \in \Lambda$, both $j_{!} k([n])$ and $j_{*}^{o} k([n])$ are $k$-vector spaces spanned by $\Lambda([1],[n])$, thus regular representations of the cyclic group $\mathbb{Z} / n \mathbb{Z}$ generated by $\sigma:[n] \rightarrow[n]$; we set $B([n])=$ id $-\sigma$ : $j_{!} k([n]) \rightarrow j_{*}^{o}([n])$. One can check that these maps are compatible with all the morphisms in $\Lambda$ and do indeed patch together to a single map $B: j_{!} k \rightarrow j_{*} k$; for the details, we refer the reader to [FT2]. Thus the complex

$$
j_{*}^{o} k \stackrel{B}{\longrightarrow} j_{!} k,
$$

placed in homological degrees 0 and 1 , has homology isomorphic to the constant functor $k$ in both degrees. We denote this complex by $j^{\dagger} k$. Moreover, fix an integer $p \geq 2$ and consider the embedding $i: \Lambda_{p} \rightarrow \Lambda$; then by base change (1.9), we have $i^{*} j_{!} k \cong j_{p !} k$ and $i^{*} j_{*}^{o} k \cong j_{p *}^{o} k$. Therefore we can pull back the connecting map $B$ by the functor $i^{*}$ and obtain a complex $j_{p}^{\dagger} k \cong i^{*} j^{\dagger} k$ in the category $\operatorname{Fun}\left(\Lambda_{p}, k\right)$. For any $p \geq 1$ and for any $p$-cyclic vector space $E \in \operatorname{Fun}\left(\Lambda_{p}, k\right)$, the projection formula shows that $j_{p !} j_{p}^{*} E \cong E \otimes j_{p !} k$ and $j_{p *}^{o} j_{p}^{o *} E \cong E \otimes j_{p *}^{o} k$. Thus we can define the connecting map

$$
j_{p *}^{o} j_{p}^{o *} E \cong E \otimes j_{p *}^{o} k \stackrel{\text { id } \otimes i^{*} B}{\longrightarrow} j_{p !} j_{p}^{*} E \cong E \otimes j_{p !} k,
$$

and a complex $j_{p}^{\dagger} E \cong E \otimes j_{p}^{\dagger} k$ whose homology is $E$ in degrees 0 and 1 .

Lemma 1.10. For any cosimplicial vector space $E^{\prime} \in \operatorname{Fun}(\Delta, k)$, we have $H .\left(\Lambda_{p}, j_{p *}^{o} E^{\prime}\right)=0$. 
Proof. For any $E \in \operatorname{Fun}\left(\Lambda_{p}, k\right)$, we have an exact sequence

$$
0 \longrightarrow E \longrightarrow j_{p *}^{o} j_{p}^{o *} E \stackrel{B}{\longrightarrow} j_{p !} j_{p}^{*} E \longrightarrow E \longrightarrow
$$

Taking $E=j_{p *}^{o} E^{\prime}$, we see, by the standard dévissage and induction on $n \geq 0$, that it suffices to prove that

$$
H_{n}\left(\Lambda_{p}, j_{p !} j_{p}^{*} j_{p *}^{o} E^{\prime}\right)=H_{n}\left(\Delta^{o}, j_{p}^{*} j_{p *}^{o} E^{\prime}\right)
$$

vanishes for all $n$. To compute $j_{p}^{*} j_{p *}^{o} E^{\prime}$, we can use base change - we factorize $j_{p}^{o}$ into the composition $\Delta \rightarrow \widetilde{\Delta}_{p} \rightarrow \Lambda_{p}$ as in (1.13), we consider the fibered product $\bar{\Delta}_{p}=\widetilde{\Delta}_{p} \times_{\Lambda_{p}} \Delta^{o}$, and we have $j_{p}^{*} j_{p *}^{o} E^{\prime} \cong \iota_{p *}^{o} \iota_{p}^{*} E^{\prime}$, where $\iota_{p}^{o}: \bar{\Delta}_{p} \rightarrow \Delta^{o}$, $\iota_{p}: \bar{\Delta}_{p} \rightarrow \widetilde{\Delta}_{p}$ are the natural projections.

We claim that for any $E^{\prime \prime} \in \operatorname{Fun}\left(\bar{\Delta}_{p}, k\right)$, we have $H_{.}\left(\Delta^{o}, \iota_{p *}^{o} E^{\prime \prime}\right)=0$

Indeed, the category $\bar{\Delta}_{p}$ is by definition equivalent to the disjoint union of $p$ copies of the category of finite linearly ordered sets equipped with a distinguished element. This category is in turn equivalent to $\Delta_{+} \times \Delta_{+}$(cut the ordered set in two at the distinguished element). By the Dold-Kan equivalence, see Subsection 1.2, $\operatorname{Fun}\left(\Delta_{+} \times \Delta_{+}, k\right)$ is equivalent to the category of bigraded $k$-vector spaces. Thus $\operatorname{Fun}\left(\bar{\Delta}_{p}, k\right)$ is semisimple, and any $E^{\prime \prime} \in \operatorname{Fun}\left(\bar{\Delta}_{p}, k\right)$ is a direct summand of a sum of corepresentable objects. Thus we may assume that $E^{\prime \prime}$ itself is corepresentable. Then so is $\iota_{p *}^{o} E^{\prime \prime} \in \operatorname{Fun}\left(\Delta^{o}, k\right)$. It remains to notice that for any representable $M \in \operatorname{Fun}\left(\Delta^{o}, k\right)$, the standard complex which by Lemma 1.1 computes $H_{\bullet}\left(\Delta^{o}, M\right)$ is acyclic.

In particular, for any $E \in \operatorname{Fun}\left(\Lambda_{p}, k\right)$ we have $H C .\left(j_{p *}^{o} j_{p}^{o *} E\right)=0$, and

$$
H_{\bullet}\left(\Lambda_{p}, j_{p}^{\dagger} E\right) \cong H_{\bullet}\left(\Lambda_{p}, j_{p !} j_{p}^{*} E\right)
$$

where the right-hand side is in turn tautologically isomorphic to $H H_{\bullet}(E)$. On the other hand, by definition we have an exact triangle

$$
E[1] \longrightarrow j_{p}^{\dagger} E \longrightarrow E \longrightarrow
$$

in $\mathcal{D}^{b}\left(\Lambda_{p}, E\right)$. Taking the homology, we obtain a long exact sequence

$$
H C_{\bullet-1}(E) \longrightarrow H_{\bullet}(E) \longrightarrow H C .(E) \longrightarrow \text {, }
$$

the transposition of the Connes' exact sequence. 
Moreover, stitching together infinitely many copies of the complex $j_{p}^{\dagger} E$, we obtain a canonical resolution of the object $E \in \operatorname{Fun}\left(\Lambda_{p}, E\right)$ :

$$
\cdots \longrightarrow j_{p *}^{o} j_{p}^{o *} E \longrightarrow j_{p !} j_{p}^{*} E \longrightarrow j_{p *}^{o} j_{p}^{o *} E \longrightarrow j_{p !} j_{p}^{*} E .
$$

Definition 1.11. The Hodge filtration on the complex (1.16) is the increasing filtration obtained by taking the even-degree terms of the stupid filtration.

Thus the associated graded quotients with respect to the Hodge filtrations are isomorphic to $j_{p}^{\dagger} E[2 l], l \geq 0$, and the filtration induces a spectral sequence

$$
\bigoplus_{l} H H_{\cdot-2 l}(E) \Rightarrow H C \cdot(E) \text {. }
$$

This is called the Hodge spectral sequence of the p-cyclic object $E$.

Dualizing Lemma 1.10, we can use the complex $j_{p}^{\dagger} k$ to compute the cohomology algebra $H^{\bullet}\left(\Lambda_{p}, k\right)$. The result is the following (the proof is immediate and left to the reader).

Lemma 1.12. We have $H^{\bullet}\left(\Lambda_{p}, k\right)=k[u]$, the polynomial algebra in one generator $u$ of degree 2 . The complex $j_{p}^{\dagger} k$ is the Yoneda representation of the generator $u \in H^{2}\left(\Lambda_{p}, k\right)=\operatorname{Ext}^{2}(k, k)$.

The natural action of the generator $u \in H^{2}\left(\Lambda_{p}, k\right)$ on cyclic homology $H C$. $(E)$ gives the periodicity map. For brevity, we will from now on use notation of the type $H H_{\text {. }}(E)\left[u^{-1}\right]$ for expressions like the left-hand side of (1.17) (with the meaning "polynomials with coefficients in $H H_{.}(E)$ in one formal variable $u^{-1}$ of homological degree 2").

Periodicity allows to introduce a version of the cyclic homology known as periodic cyclic homology.

Definition 1.13. The periodic cyclic homology $H P .(E)$ of a $p$-cyclic vector space $E \in \operatorname{Fun}\left(\Lambda_{p}, E\right)$ is defined as the inverse limit

$$
H P .(E)=\lim _{\leftarrow} \bullet H C .(E)
$$

with respect to the periodicity map. 
Here $\lim ^{\bullet}$ is the derived functor of the inverse limit lim; explicitly, it can be computed by the telescope construction - that is, $\lim _{\underline{u}} \cdot H C$. $(E)$ is the cone of the map

$$
H_{\text {. }}(E)[[t]] \stackrel{\text { id }-t u}{\longrightarrow} H C_{.}(E)[[t]],
$$

where $H C_{.}(E)[[t]]=\prod_{i \geq 0} H C$. $(E)[-2 i]$ means "formal power series with coefficients in $H C .(E)$ in one formal variable $t$ of degree -2 ". In practice, in our applications all the cyclic objects will have non-trivial Hochschild homology only in a finite range of degrees, so that the inverse system stabilizes in every degree, and $H P .(E)=\lim _{\leftarrow} H C$. $(E)$.

1.7. Homological effects of the $p$-fold coverings. We will now fix an integer $p \geq 2$ and describe the homological properties of the functors in the diagram (1.12). For the horizontal embedding $i: \Lambda_{p} \rightarrow \Lambda$, the situation is extremely nice.

Lemma 1.14. For any cyclic $k$-vector space $E \in \operatorname{Fun}(\Lambda, k)$, the natural adjunction maps

$$
H C .\left(i^{*} E\right) \rightarrow H C .(E), \quad H P .\left(i^{*} E\right) \rightarrow H P .(E)
$$

are isomorphisms.

L. Hesselholt informed me that this is actually a completely standard fact known as the Cyclic Subdivision Lemma (and valid not only for vector spaces, but for abelian groups and, with appropriate modifications, for topological spaces). For the convenience of the reader, we include a sketch of a proof adapted from [Ka, Proposition 1.7].

Sketch of a proof. Since by definition $i^{*} j^{\dagger} k \cong j_{p}^{\dagger} k$, the adjunction map is compatible with periodicity; therefore by the usual dévissage using (1.15), it suffices to prove that the map is an isomorphism on the level of Hochschild homology. By base change, it moreover suffices to prove that for any $E \in \operatorname{Fun}\left(\Delta^{o}, k\right)$, the adjunction map

$$
H_{.}\left(\Delta^{o}, i^{*} E\right) \rightarrow H_{\bullet}\left(\Delta^{o}, E\right)
$$

is an isomorphism, where $i$ is now the functor $\Delta \rightarrow \Delta$ sending the linearly ordered set $[n]$ to $[n p]=[n] \times[p]$ (with the lexicographical order on $[n] \times[p]$ ). It is enough to prove this for one generator $E$ of the category $\operatorname{Fun}\left(\Delta^{o}, k\right)$. Let $B=k[t]$ be the polynomial algebra in one variable $t$, and let $S \in \operatorname{Fun}\left(\Delta^{o}, k\right)$ be the standard bar resolution of the diagonal $B$-bimodule $B$, with the standard structure of a 
simplicial vector space. The polynomial algebra $B$ is graded by the degree of the polynomial; this induces a grading on $S$, and one checks easily that the $l$-th graded component $S_{l} \in \operatorname{Fun}\left(\Delta^{o}, k\right)$ is precisely the standard $l+1$-simplex - that is, the $k$-linear span of the functor $\Delta^{o} \rightarrow$ Sets represented by $[l+1]\left(S_{l}([n])\right.$ is the space of all polynomials of degree $l$ on $n+1$ variables, and this is exactly the $k$-vector space spanned by $\left.\Delta^{o}([l+1],[n])\right)$. Therefore $S$ is a generator of $\operatorname{Fun}\left(\Delta^{o}, k\right)$. On the other hand, it is also easy to check that

$$
i^{*} S \cong S \otimes_{B} S \otimes_{B} \cdots \otimes_{B} S,
$$

with $p$ copies of $S$ on the right-hand side, and the tensor product taken in $\operatorname{Fun}\left(\Delta^{o}, k\right)$. Therefore $i^{*} S$ is a simplicial resolution of $B \otimes_{B} B \otimes_{B} \cdots \otimes_{B} B$, which is identified with $B$ by the multiplication map $B \otimes_{B} B \otimes_{B} \cdots \otimes_{B} B \rightarrow B$. By Lemma 1.1, this means that the natural map $H_{\bullet}\left(\Delta^{o}, i^{*} S\right) \rightarrow H_{\bullet}\left(\Delta^{o}, S\right)$ is indeed a quasiisomorphism, with both sides quasiisomorphic to $B$.

If the base field $k$ has characteristic 0, then the vertical map in (1.12), the projection $\pi: \Lambda_{p} \rightarrow \Lambda$, enjoys a similar property. However, it is no longer true if the characteristic is positive and divides $p$. Assume from now on that $p \geq 2$ is prime, and that char $k=p$.

Lemma 1.15. For any bifibration $\sigma: \Gamma^{\prime} \rightarrow \Gamma$ with fiber $\mathrm{pt}_{p}$, and any $E \in$ $\operatorname{Fun}\left(\Gamma^{\prime}, k\right)$, the following conditions are equivalent.

(i) $L^{i} \sigma_{!} E=0$ for $i \geq 1$.

(ii) $R^{i} \sigma_{*} E=0$ for $i \geq 1$.

(iii) The natural trace map $\operatorname{tr}: \sigma_{!} E \rightarrow \sigma_{*} E$ is an isomorphism.

(iv) for any $[a] \in \Gamma^{\prime}, E([a])$ is a free $\mathbb{Z} / p \mathbb{Z}$-module (with respect to the natural $\mathbb{Z} / p \mathbb{Z}$-action given by the identification $\left.\sigma_{\sigma([a])} \cong \mathrm{pt}_{p}\right)$.

Proof. Since $\sigma$ is a bifibration with finite fibers, we can apply base change and assume, without any loss of generality, that $\Gamma=\mathrm{pt}$, so that $\Gamma^{\prime}=\mathrm{pt}_{p}, E$ is a $k[\mathbb{Z} / p \mathbb{Z}]$-module, and $L^{*} \sigma_{!} E, R^{*} \sigma_{*} E$ are its homology and cohomology. Then obviously (iv) implies (i) and (ii), and conversely, it is easy to check that either of (i) or (ii) implies (iv). The equivalence (iii) $\Leftrightarrow$ (iv) is also obvious: since char $k=p$, we have $k[\mathbb{Z} / p \mathbb{Z}]=k[t] / t^{p}$, the truncated polynomial algebra, we have $H_{0}(\mathbb{Z} / p \mathbb{Z}, E)=E / t, H^{0}(\mathbb{Z} / p \mathbb{Z}, E) \subset E$ is the kernel of the multiplication by $t$, the natural trace map $H_{0}(\mathbb{Z} / p \mathbb{Z}, E) \rightarrow H^{0}(\mathbb{Z} / p \mathbb{Z}, E)$ is the map $E / t \rightarrow \operatorname{Ker} t \subset E$ 
induced by multiplication by $t^{p-1}$, and it is invertible if and only if $E$ is a free module over $k[t] / t^{p}$.

Lemma 1.16. For any $E \in \operatorname{Fun}(\Lambda, k)$, we have

$$
H C_{.}\left(\pi^{*} E\right) \cong H_{.}(E)\left[u^{-1}\right], \quad H P_{.}\left(\pi^{*} E\right) \cong H H_{\bullet}(E)((u)) .
$$

Moreover, the restriction map $\pi^{*}: H^{2}(\Lambda, k) \rightarrow H^{2}\left(\Lambda_{p}, k\right)$ is trivial.

Proof. Apply Lemma 1.15 to the bifibration $\pi: \Lambda_{p} \rightarrow \Lambda$. One checks immediately that the functors $j ! k, j_{*}^{o} k \in \operatorname{Fun}\left(\Lambda_{p}, k\right)$ satisfy the condition (iv), hence also (i). Therefore we can compute $L^{\bullet} \pi ! k$ by means of the resolution (1.16). This gives a complex with even-degree terms $\pi_{!} j_{p !} k$ and odd-degree terms $\pi_{!} j_{p *}^{o} k$. Since $\pi \circ j_{p}=j, \pi \circ j_{p}^{o}=j^{o}$, we have $\pi_{!} j_{p !} k \cong j_{!} k$, and by Lemma 1.15 (iii), $\pi_{!} j_{p *}^{o} k \cong$ $\pi_{*} j_{p *}^{o} \cong j_{*}^{o} k$. Moreover, the differential $j_{p !} k \rightarrow j_{p *}^{o} k$ in the resolution (1.16) factors as $j_{p !} k \rightarrow k \rightarrow j_{p *}^{o} k$, and we have a commutative diagram

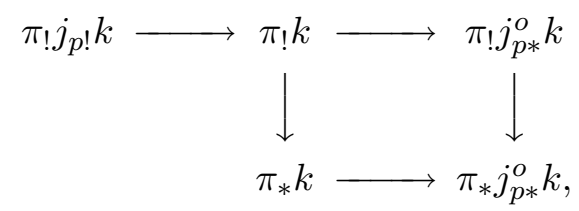

where the vertical map on the right-hand side is the isomorphism $\pi_{!} j_{p *}^{o} k \cong \pi_{*} j_{p *}^{o} k$. But $k([n])=k$ is the trivial $\mathbb{Z} / p \mathbb{Z}$-module for any $[n] \in \Lambda_{p}$, and one immediately checks that the trace map $\operatorname{tr}: \pi_{!} k \rightarrow \pi_{*} k$ is zero. Therefore the differentials $j ! k \rightarrow j_{*}^{o} k$ in the complex that computes $L^{\bullet} \pi_{!} k$ are trivial, so that the complex splits into a direct sum of pieces of the form $j_{*}^{o} k \rightarrow j_{!} k$. We now substitute this complex into the projection formula to compute $L^{\bullet} \pi_{!} \pi^{*} E$; by Lemma 1.10, $H C .\left(E \otimes j_{*}^{o} k\right)=0$, and we conclude that

$$
H C .\left(\pi^{*} E\right) \cong H C .\left(L^{\bullet} \pi_{!} \pi^{*} E\right) \cong \bigoplus_{n} H C .(E \otimes j ! k[2 n])=H H_{\bullet}\left[u^{-1}\right] .
$$

Passing to the limit with respect to the periodicity morphism $u$, we get the second claim. Finally, the first claim shows that the periodicity element $u_{0} \in H^{2}(\Lambda, k)$ acts trivially on $H$. $\left(\Lambda_{p}, k\right) \cong k\left[u^{-1}\right]$; this implies that $\pi^{*} u_{0}=0$.

Remark 1.17. In algebraic topology, and specifically in Topological Cyclic Homology, another approach to cyclic homology is commonly used: instead of defining a cyclic object, which contains both Hochschild and cyclic homology in one package, one first defines Hochschild homology in some way, and then equips 
it with a $U(1)$-equivariant structure understood in the appropriate sense - for instance, in the sense of equivariant homotopy theory and the category of $U(1)$ equivariant spectra. One reconstructs cyclic homology using this $U(1)$-action (for the usual cyclic homology, this approach is described, for instance, in [L, Chapter 7]). The category $\Lambda$ is thus essentially replaced with its topological realization, which can be naturally identified with the classifying space $B U(1)$ of the circle $U(1)=S^{1}$; cyclic objects are replaced with sheaves on $B U(1)$. In this sense, our projection $\pi: \Lambda_{p} \rightarrow \Lambda$ corresponds to the $p$-fold covering $U(1) \rightarrow U(1), z \mapsto z^{p}$. Lemma 1.16 describes the homological properties of the correponding fibration $B U(1) \rightarrow B U(1)$.

1.8. Relation to group homology. We note that for any $E \in \operatorname{Fun}\left(\Lambda_{p}, k\right)$, we tautologically have

$$
H C .(E) \cong H C .\left(L^{\bullet} \pi_{!} E\right) \text {; }
$$

however, this isomorphism is not compatible with the natural periodicity maps on both sides - in fact, as Lemma 1.16 shows, the periodicity map on the righthand side is equal to 0 . To study the periodic cyclic homology HP. $(E)$ in terms of $L^{\bullet} \pi ! E$, we use group homology. We still assume that char $k=p$.

Recall that for any finite group $G$ and any $G$-module $V$, one can use the trace map tr: $H_{0}(G, V) \rightarrow H^{0}(G, V)$ to stitch together the homology $H_{\text {. }}(G, V)$ and the cohomology $H^{\bullet}(G, V)$ and obtain the so-called Tate homology $\check{H}_{\bullet}(G, V)$. This is a certain (unbounded) cohomological functor from $G$-modules to vector spaces. To compute it, one uses the fact that a $G$-module $V$ is injective if and only if it is projective, and if this happens, the trace map $H_{0}(G, V) \rightarrow H^{0}(G, V)$ is an isomorphism and $\breve{H}_{.}(G, V)=0$ in all degrees. One then takes a projective resolution $P$. and an injective resolution $I^{\bullet}$ of the trivial $G$-module $k$, and stitches them together to an (unbounded acyclic) complex $\check{P}_{\text {. }}$; for any $G$-module $V$, one has

$$
\check{H}^{\bullet}(G, V) \cong H_{0}\left(G, V \otimes \check{P}_{.}\right) \cong H^{0}\left(G, V \otimes \check{P}_{.}\right) .
$$

In the particular case $G=\mathbb{Z} / p \mathbb{Z}$, the second cohomology group $H^{2}(\mathbb{Z} / p \mathbb{Z}, k)$ is one-dimensional; any non-trivial element $u \in H^{2}(\mathbb{Z} / p \mathbb{Z}, k)$ induces a periodicity endomorphism on homology $H$. $(\mathbb{Z} / p \mathbb{Z}, V)$. Thus any non-split exact sequence

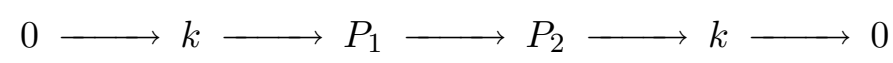


of $\mathbb{Z} / p \mathbb{Z}$-modules represents by Yoneda a class which generates $H^{2}(\mathbb{Z} / p \mathbb{Z}, k)$. If $P_{1}$ and $P_{2}$ are free, the sequence cannot be split. Iterating it as in (1.16), we obtain a periodic free resolution $P$. of the trivial $\mathbb{Z} / p \mathbb{Z}$-module $k$; iterating in both directions gives a Tate resolution $\check{P}$., and therefore

$$
\check{H}_{\bullet}(\mathbb{Z} / p \mathbb{Z}, V) \cong \lim _{\underline{u}} \cdot H_{\bullet}(\mathbb{Z} / p \mathbb{Z}, V)
$$

for any $V$ and any generator $u \in H^{2}(\mathbb{Z} / p \mathbb{Z}, k)$.

In particular, consider the embedding $\bar{j}_{p}: \Delta^{o} \times \mathrm{pt}_{p} \rightarrow \Lambda_{p}$. Since evaluating the complex $j_{p}^{\dagger} k$ at any $[n] \in \Lambda_{p}$ gives a complex of free $k[\mathbb{Z} / p \mathbb{Z}]$-modules, $j^{\dagger}([n])$ represents a non-trivial class in $\operatorname{Ext}_{\mathbb{Z} / p \mathbb{Z}}^{2}(k, k)$, and the restriction map

$$
\bar{j}_{p}^{*}: H^{\bullet}\left(\Lambda_{p}, k\right) \rightarrow H^{\bullet}\left(\Delta^{o} \times \mathrm{pt}_{p}, k\right)=H^{\bullet}\left(\Delta^{o}, k\right) \otimes H^{\bullet}\left(\mathrm{pt}_{p}, k\right)=H^{\bullet}(\mathbb{Z} / p \mathbb{Z}, k)
$$

sends the periodicity element $u \in H^{2}\left(\Lambda_{p}, k\right)$ into a generator of $H^{2}(\mathbb{Z} / p \mathbb{Z}, k)$. Thus for any $E \in \operatorname{Fun}\left(\Lambda_{p}, k\right)$, the adjunction map $H_{\bullet}\left(\Delta^{o} \times \mathrm{pt}_{p}, \bar{j}_{p}^{*} E\right) \rightarrow H C$. $(E)$ extends to a map

$$
\lim _{\leftarrow} H_{\bullet}\left(\Delta^{o} \times \mathrm{pt}_{p}, \bar{j}_{p}^{*} E\right) \rightarrow H P .(E) .
$$

Moreover, the left-hand side is isomorphic to the Tate homology

$$
\lim _{\stackrel{u}{\leftarrow}} H_{\cdot}\left(\Delta^{o} \times \mathrm{pt}_{p}, \bar{j}_{p}^{*} E\right) \cong \check{H}\left(\mathbb{Z} / p \mathbb{Z}, L^{\cdot} \tau_{!} \bar{j}_{p}^{*} E\right),
$$

where $\tau: \Delta^{o} \rightarrow$ pt is the projection to the point, and we treat $\bar{j}_{p}^{*} E$ as an object in $\operatorname{Fun}\left(\Delta^{o}, k[\mathbb{Z} / p \mathbb{Z}]\right.$ mod $)$. Using the base change isomorphism for the diagram (1.14), we can identify $H_{\bullet}\left(\Delta^{o} \times \mathrm{pt}_{p}, \bar{j}_{p}^{*} E\right)$ with $H_{\bullet}\left(L^{\bullet} \pi_{!} E\right)$; then the exact triangle (1.15) extends (1.19) to a long exact sequence

$$
H P_{\bullet+1}(E) \longrightarrow \check{H}\left(\mathbb{Z} / p \mathbb{Z}, L^{\bullet} \tau_{!} \bar{j}_{p}^{*} E\right) \longrightarrow H P .(E) \longrightarrow,
$$

where the connecting differential is equal to 0 by Lemma 1.16. We use this to obtain the following vanishing result.

Definition 1.18. An object $E$. $\in \mathcal{D}^{b}\left(\Lambda_{p}, k\right)$ is said to be small if its restriction $\bar{j}_{p}^{*} E . \in \mathcal{D}^{b}\left(\operatorname{Fun}\left(\Delta^{o}, k[\mathbb{Z} / p \mathbb{Z}] \bmod \right)\right)$ is effectively finite in the sense of Definition 1.2.

Lemma 1.19. For any small $E \in \operatorname{Fun}\left(\Lambda_{p}, k\right)$ which satisfies the equivalent conditions of Lemma 1.15 for the bifibration $\pi: \Lambda_{p} \rightarrow \Lambda$, we have $H P .(E)=0$. 
Proof. By (1.20), it suffices to prove that

$$
\check{H}\left(\mathbb{Z} / p \mathbb{Z}, L^{\cdot} \tau_{!} E^{\prime}\right)=0,
$$

where $E^{\prime}=\bar{j}_{p}^{*} E$ is the simplicial $k[\mathbb{Z} / p \mathbb{Z}]$-module obtained by restriction from $E \in \operatorname{Fun}\left(\Lambda_{p}, k\right)$. By assumption, the $\mathbb{Z} / p \mathbb{Z}$-module $E([n])$ is free, hence projective and injective, for any $[n] \in \Lambda_{p}$. Consider the stupid filtration $F . E^{\prime}$ and the quotients $F^{\bullet} E^{\prime}$. For any integer $n \geq 1$, we can compute $L^{\bullet} \tau_{!} F_{n} E^{\prime}$ by the standard complex, which in this case is a finite complex of free $k[\mathbb{Z} / p \mathbb{Z}]$-modules. Therefore

$$
\check{H}\left(\mathbb{Z} / p \mathbb{Z}, L^{\bullet} \tau_{!} F_{i} E^{\prime}\right)=0
$$

for any $n \geq 1$. Since $E$ is small, $E^{\prime}$ is effectively finite; in particular, for some $n \geq 1$ the second map in the exact triangle

$$
L^{\bullet} \tau_{!} F_{n} E^{\prime} \longrightarrow L^{\bullet} \tau_{!} E^{\prime}=L^{\bullet} \tau_{!} F^{0} E^{\prime} \longrightarrow L^{\bullet} \tau_{!} F^{n} E^{\prime} \longrightarrow
$$

is equal to 0 . Tate homology sends this exact triangle into an exact triangle, and the map which was trivial stays trivial; therefore the map

$$
\check{H}_{m}\left(\mathbb{Z} / p \mathbb{Z}, L^{\cdot} \tau_{!} F_{n} E^{\prime}\right) \rightarrow \check{H}_{m}\left(\mathbb{Z} / p \mathbb{Z}, L^{\bullet} \tau_{!} E^{\prime}\right)
$$

is surjective for any $m$. This finishes the proof.

\section{Cartier map - the simple Case.}

2.1. Cyclic homology of algebras. We will now turn to the cyclic homology of algebras. For any associative unital algebra $A$ over a field $k$, one defines the functor $A_{\sharp} \in \operatorname{Fun}(\Lambda, k)$ in the following way. For any $[n] \in \Lambda$, we denote by $V([n])$ the set of vertices of the corresponding wheel (equivalently, $V([n])=\Lambda([1],[n])$. On objects, we set $A_{\sharp}([n])=A^{\otimes V([n])}$, the product of $n$ copies of the algebra $A$ numbered by the set $V([n])$. For any map $f:[m] \rightarrow[n]$, we set

$$
A_{\sharp}(f)\left(\bigotimes_{i \in V([m])} a_{i}\right)=\bigotimes_{j \in V([n])} \prod_{i \in f^{-1}(j)} a_{i}
$$

(if $f^{-1}(i)$ is empty for some $i \in V([n])$, then the right-hand side involves a product numbered by the empty set; this is defined to be the unity element $1 \in A$ ).

We note that this construction immediately generalizes to assoiative unital algebras in a unital symmetric monoidal category $\langle\mathcal{C}, \otimes\rangle$ - for any such algebra 
$A \in \mathcal{C}$, we obtain a functor $A_{\sharp}: \Lambda \rightarrow \mathcal{C}$. Moreover, we can dualize this construction - for any coalgebra $A \in \mathcal{C}$, we denote the corresponding functor by $A^{\sharp}: \Lambda^{\circ} \rightarrow \mathcal{C}$.

Definition 2.1. For any associative unital algebra $A$ over the field $k$, the Hochschild, cyclic and periodic cyclic homology of the algebra $A$ is defined by

$$
H H_{\bullet}(A)=H H_{\bullet}\left(A_{\sharp}\right), \quad H C .(A)=H C .\left(A_{\sharp}\right), \quad H P .(A)=H P .\left(A_{\sharp}\right) .
$$

Remark 2.2. It is well-known that the Hochschild homology can be more invariantly defined as $H H_{.}(A)=\operatorname{Tor}_{A^{\circ} \otimes A}(A, A)$; to obtain the description above, one computes Tor ${ }^{\bullet}$ by using the bar resolution. In this paper, we will not need this description, except for one place in Section 5. An analogous invariant description for $H C$. $(A)$ or $H P$. $(A)$ is not known.

If $A$ is a finitely generated commutative algebra, and $\operatorname{Spec} A$ is smooth, then in has been known since [HKR] that the Hochschild homology groups are naturally identified with differential forms on $\operatorname{Spec} A$ - for any $i \geq 0$, we have $H H_{i}(A) \cong$ $\Omega^{i}(A / k)$. The periodic cyclic homology $H P .(A)$ is then naturally identified with $H_{D R}^{\cdot}(A)((u))$, where $u$, as before, is a formal generator of degree 2 , and $H_{D R}^{\cdot}(A)=$ $H_{D R}^{\cdot}(\operatorname{Spec} A)$ are the de Rham cohomology groups of the scheme Spec $A$ (over the base field $k$ ). This, in particular, motivates the term Hodge spectral sequence for (1.17). For more details on relation between cyclic homology and differential forms see [L, Chapter 2.3].

Assume from now on, and until Section 5, that the base field $k$ is a perfect field of positive characteristic char $k=p>0$. For any $k$-vector space $V$, denote by $V^{(1)}$ the Frobenius twist of $V$ - that is, the pullback $\operatorname{Fr}^{*} V$ with respect to the Frobenius map $\mathrm{Fr}: k \rightarrow k$. Again assume for a moment that we are within the assumptions of the Hochschild-Kostant-Rosenberg Theorem - $A$ is finitely generated and commutative, and $\operatorname{Spec} A$ is smooth. Moreover, assume that $\operatorname{dim} A<p$. Then it was discovered by P. Cartier back in the 1950ies that there exists a natural isomorphism

$$
C: H_{D R}^{\bullet}(A) \cong \Omega^{\bullet}\left(A^{(1)}\right)
$$

which identifies de Rham cohomology classes of $A$ with differential forms on $A^{(1)}$. It will be more convenient for us to consider the inverse isomorphism $C^{-1}: \Omega^{\bullet}\left(A^{(1)}\right) \rightarrow H_{D R}^{\bullet}(A)$. Using the identifications $H P .(A) \cong H_{D R}^{\cdot}(A)((u))$, 
$H H_{\bullet}\left(A^{(1)}\right) \cong \Omega^{\bullet}\left(A^{(1)}\right)$, it can be rewritten as an isomorphism

$$
C^{-1}: H_{.}\left(A^{(1)}\right)((u)) \cong H P .(A) .
$$

It turns out that such an isomorphism actually exists for a much wider class of algebras $A$, and in particular, $A$ does not have to be commutative (in fact, with certain modification, the inverse Cartier map can be defined for any associative unital algebra whatsoever, and in a functorial way). We will explain this in detail in Section 4. In this Section, we will illustrate the general methods by constructing an isomorphism (2.2) under an additional assumption on $A$. Namely, we note the following.

Lemma 2.3. For any vector space $V$ over $k$ and any integer $i$, we have a natural identification

$$
V^{(1)} \cong \check{H}_{i}^{(1)}\left(\mathbb{Z} / p \mathbb{Z}, V^{(1)}\right) \cong \check{H}_{i}\left(\mathbb{Z} / p \mathbb{Z}, V^{\otimes p}\right),
$$

where the Tate homology in the right-hand side is taken with respect to the natural permutation action of $\mathbb{Z} / p \mathbb{Z}$ on the tensor self-product $V^{\otimes p}$, and the $\mathbb{Z} / p \mathbb{Z}$-action on $V^{(1)}$ in the middle term is trivial.

Proof. For any $k[\mathbb{Z} / p \mathbb{Z}]$-module $E$, we can compute the Tate homology $\check{H}^{\bullet}(\mathbb{Z} / p \mathbb{Z}, E)$ using the standard periodic complex with terms $E$ and differentials $d_{i}$,

$$
d_{i}= \begin{cases}\mathrm{id}-\sigma, & i \text { is odd, } \\ \mathrm{id}+\sigma+\cdots+\sigma^{p-1} & i \text { is even, }\end{cases}
$$

where $\sigma$ is the generator of the group $\mathbb{Z} / p \mathbb{Z}$. When $E=V^{(1)}$ with trivial $\mathbb{Z} / p \mathbb{Z}$-action, these differential are obviously equal to zero, so that indeed $V^{(1)} \cong$ $\check{H}_{.}^{(1)}\left(\mathbb{Z} / p \mathbb{Z}, V^{(1)}\right)$ in every degree. Let $E=V^{\otimes p}$. Then the map $\tau: V^{(1)} \rightarrow V^{\otimes p}$, $v \mapsto v \otimes v \otimes \cdots \otimes v$ is compatible with the multiplication by scalars, and it sends $V$ into the kernel of differential $d_{i}: V^{\otimes p} \rightarrow V^{\otimes p}$, both for odd and even indices $i$. We claim that it is additive "modulo $\operatorname{Im} d_{i+1}$ ", and that it induces an isomorphism $V^{(1)} \cong \operatorname{Ker} d_{i} / \operatorname{Im} d_{i+1}$. Indeed, choose a basis in $V$, so that $V \cong k[S]$, the $k$-linear span of a set $S$. Then $V^{\otimes p}=k\left[S^{p}\right]$ decomposes as $k\left[S^{p}\right]=k[S] \oplus k\left[S^{p} \backslash \Delta\right]$, where $S \cong \Delta \subset S^{p}$ is the diagonal. This decomposition is compatible with the differentials $d_{i}$, which actually vanish on the first summand $k[S] \cong V^{(1)}$. The map $\tau$, accordingly, decomposes as $\tau=\tau_{0} \oplus \tau_{1}, \tau_{0}: V^{(1)} \rightarrow k[S], \tau_{1}: V^{(1)} \rightarrow k\left[S^{p} \backslash \Delta\right]$. The map $\tau_{0}$ is obviously additive and an isomorphism; therefore it suffices to 
prove that the second summand of the periodic complex is acyclic. Indeed, since the $\mathbb{Z} / p \mathbb{Z}$-action on $S^{p} \backslash \Delta$ is free, we have $\check{H}^{\bullet}\left(\mathbb{Z} / p \mathbb{Z}, k\left[S^{p} \backslash \Delta\right]\right)=0$.

Definition 2.4. A quasi-Frobenius map for an associative unital algebra $A$ over $k$ is a $\mathbb{Z} / p \mathbb{Z}$-equivariant algebra map $F: A^{(1)} \rightarrow A^{\otimes p}$ which induces an isomorphism $\check{H}_{.}\left(\mathbb{Z} / p \mathbb{Z}, A^{(1)}\right) \rightarrow \check{H}$. $\left(\mathbb{Z} / p \mathbb{Z}, A^{\otimes p}\right)$.

Here the $\mathbb{Z} / p \mathbb{Z}$-action on $A^{(1)}$ is trivial, and the algebra structure on $A^{\otimes p}$ is the obvious one (all the $p$ factors commute). We note that since $\check{H}_{i}(\mathbb{Z} / p \mathbb{Z}, k) \cong k$ for every $i$, we have $\check{H}_{i}\left(\mathbb{Z} / p \mathbb{Z}, A^{(1)}\right) \cong A^{(1)}$, so that a quasi-Frobenius map must be injective. Moreover, since the Tate homology $\check{H}_{\bullet}\left(\mathbb{Z} / p \mathbb{Z}, A^{\otimes p} / A^{(1)}\right)$ vanishes, the cokernel of a quasi-Frobenius map must be a free $k[\mathbb{Z} / p \mathbb{Z}]$-module.

2.2. Polycyclic objects and the inverse Cartier map. In the remainder of this Section, we will construct an inverse Cartier isomorphism (2.2) for an algebra $A$ which admits a quasi-Frobenius map. In the interest of full disclosure, we must warn the reader that this almost never happens. In fact, the only example which comes to mind is the free associative algebra $A=T^{\bullet} V$ generated by a vector space $V$, and more generally, the group algebra $A=k[G]$ of some discrete group $G$; and even in this case, constructing a quasi-Frobenius map uses the fixed basis in $k[G]$, so that the map can not be made functorial with respect to algebra maps. Nevertheless, it is only the notion of a quasi-Frobenius map that we will modify and extend in Chapter 4; once it has been done, the construction of the Cartier isomorphism remains the same.

Remark 2.5. The name "quasi-Frobenius" is somewhat misleading: even if the algebra $A$ is commutative, and even if it admits a quasi-Frobenius map $\widetilde{F}$, this map $\widetilde{F}$ has nothing to do with the Frobenius map in the usual sense (in fact, it is closer for the Verschiebung map $V$ which exists for commutative group schemes)

Every set $X$ is tautologically a coalgebra in the symmetric monoidal category of sets (with the diagonal map as comultiplication). The same is true for the category of (small) categories. Thus for any small category $C$, we can form the functor $C^{\sharp}: \Lambda^{o} \rightarrow$ Cat. Applying the Grothendieck construction to this functor, as in Subsection 1.4, we obtain a category fibered over $\Lambda$ which we call the wreath product of $\Lambda$ and $C$ and denote by $C \int \Lambda$. One immediately generalizes 
the $\sharp$-construction so that for any associative algebra $A$ in $\operatorname{Fun}(C, k)$ with respect to the pointwise tensor structure, we obtain an object $A_{\sharp}$ in $\operatorname{Fun}\left(C \int \Lambda, k\right)$ (the structure maps are given by exactly the same formula (2.1) as in the case $C=\mathrm{pt}$ ).

In particular, let $C=\mathrm{pt}_{p}$, the groupoid with one object whose automorphism group is $\mathbb{Z} / p \mathbb{Z}$.

Definition 2.6. The polycyclic category $\mathrm{B}_{p}$ is the wreath product of $\Lambda$ and $\mathrm{pt}_{p}$, $\mathrm{B}_{p}=\mathrm{pt}_{p} \int \Lambda$.

Thus by definition, $\mathrm{B}_{p}$ is equiped with a fibration $\chi: \mathrm{B}_{p} \rightarrow \Lambda$ whose fiber over $[n] \in \Lambda$ is the category $\mathrm{pt}_{p}^{n}$ (that is, the groupoid with one object and automorphism group $\left.(\mathbb{Z} / p \mathbb{Z})^{n}\right)$. Explicitly, $\mathrm{B}_{p}$ has the same objects as $\Lambda$, and a map $\tilde{f} \in \mathrm{B}_{p}([m],[n])$ is a pair $\langle f, h\rangle$ of a map $f \in \Lambda([m],[n])$ and an element $h \in(\mathbb{Z} / p \mathbb{Z})^{m}$; the composition with some $\left\langle f^{\prime}, h^{\prime}\right\rangle \in \mathrm{B}_{p}([n],[l])$ is given by

$$
\langle f, h\rangle \circ\left\langle f^{\prime}, h^{\prime}\right\rangle=\left\langle f \circ f^{\prime}, h+f^{*}\left(h^{\prime}\right)\right\rangle,
$$

where $f^{*}:(\mathbb{Z} / p \mathbb{Z})^{n} \rightarrow(\mathbb{Z} / p \mathbb{Z})^{m}$ is the map corresponding to the functor $\left(\mathrm{pt}_{p}\right)_{\sharp}$ : $\Lambda^{o} \rightarrow$ Cat. To see $f^{*}$ explicitly, we can use the combinatorial description of $\Lambda$ given in Subsection $1.5-\mathrm{Cycl}$ is the category of linearly ordered sets equipped with an order-preserving automorphism $\sigma$, for any $l \geq 1,[l] \in$ Cycl is the linearly ordered set $\mathbb{Z}$ with $\sigma: x \mapsto x+l$; then $(\mathbb{Z} / p \mathbb{Z})^{m}=\left(\mathrm{pt}_{p}\right)_{\sharp}([m])$ can be interpreted as the set $\operatorname{Hom}^{\sigma}([m], \mathbb{Z} / p \mathbb{Z})$ of all $\sigma$-invariant maps from $[m]=\mathbb{Z}$ to $\mathbb{Z} / p \mathbb{Z}$, and $f^{*}\left(h^{\prime}\right)=h^{\prime} \circ f:[m] \rightarrow \mathbb{Z} / p \mathbb{Z}$.

For any associative algebra $B$ over $k$ equipped with an action of the group $\mathbb{Z} / p \mathbb{Z}$ - in particular, for $B=A^{\otimes p}, A$ an associative algebra over $k$ - we have a canonical object $B_{\sharp} \in \operatorname{Fun}\left(\mathrm{B}_{p}, k\right)$.

Lemma 2.7. There exists an embedding $\lambda: \Lambda_{p} \rightarrow \mathrm{B}_{p}$ which induces the diagonal embedding $\mathrm{pt}_{p} \rightarrow \mathrm{pt}_{p}^{n}$ on the fiber over $[n] \in \Lambda$ and fits into a commutative diagram

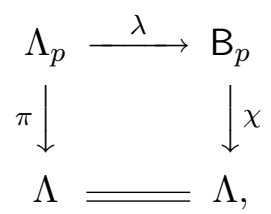

where $\pi: \Lambda_{p} \rightarrow \Lambda$ is as in (1.12). For any associative $k$-algebra $A$, we have $\lambda^{*}\left(A^{\otimes p}\right)_{\sharp} \cong i^{*} A_{\sharp}$, where $i: \Lambda_{p} \rightarrow \Lambda$ is the embedding from (1.12). 
Proof. Let $\mathrm{B}_{\infty}=\mathrm{pt}_{\infty} \int \Lambda$, where $\mathrm{pt}_{\infty}$ is the groupoid with one object whose automorphism group is $\mathbb{Z}$, and let $\widetilde{\mathrm{B}}_{\infty}=\Lambda_{\infty} \times_{\Lambda} \mathrm{B}_{\infty}$, so that we have a Cartesian square

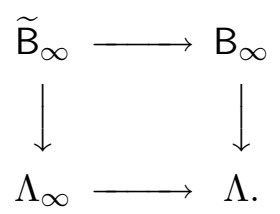

Explicitly, objects of $\widetilde{\mathrm{B}}_{\infty}$ are $[n], n \geq 1$, and for any $[n],[m]$ the set of morphisms from $[m]$ to $[n]$ is given by

$$
\begin{aligned}
\widetilde{\mathrm{B}}_{\infty}([m],[n]) & =\mathbb{Z}^{m} \times \Lambda_{\infty}([m],[n]) \\
& =\left\{\left\langle f^{\prime}, f\right\rangle \mid f^{\prime} \in \operatorname{Hom}^{\sigma}([m], \mathbb{Z}), f \in \operatorname{Hom}_{\mathrm{Cycl}}([m],[n])\right\},
\end{aligned}
$$

with composition defined by $\left\langle g^{\prime}, g\right\rangle \circ\left\langle f^{\prime}, f\right\rangle=\left\langle\left(g^{\prime} \circ f\right)+f^{\prime}, g \circ f\right\rangle$. For any $f \in \operatorname{Hom}_{\mathrm{Cycl}}([m],[n])$, there obviously exists a unique $f^{\prime} \in \operatorname{Hom}^{\sigma}([m], \mathbb{Z})$ such that

- for any $l, 0 \leq l<m$, we have

$$
0 \leq f(l)+n f^{\prime}(l)<n .
$$

It is easy to see that this condition is preserved by the composition law, so that the correspondence $f \mapsto\left\langle f^{\prime}, f\right\rangle$ gives a section $\Lambda_{\infty} \rightarrow \widetilde{\mathrm{B}}_{\infty}$ of the projection $\widetilde{\mathrm{B}}_{\infty} \rightarrow \Lambda_{\infty}$ in (2.3). Composing this section with the projection $\widetilde{\mathrm{B}}_{\infty} \rightarrow \mathrm{B}_{\infty}$, we obtain a functor $\lambda_{\infty}: \Lambda_{\infty} \rightarrow \mathrm{B}_{\infty}$ compatible with the projections to $\Lambda$. Reducing this modulo $p$ gives the functor $\lambda: \Lambda_{p} \rightarrow \mathrm{B}_{p}$. Checking that $\lambda^{*}\left(A^{\otimes p}\right)_{\sharp} \cong i^{*} A_{\sharp}$ is an easy combinatorial excersize which we leave to the reader.

Lemma 2.8. Assume that the quotient $V / W$ of a $k[\mathbb{Z} / p \mathbb{Z}]$-module $V$ by a $k[\mathbb{Z} / p \mathbb{Z}]$ submodule $W$ is a free $k\left[\mathbb{Z} / p \mathbb{Z}\left[\right.\right.$-module. Then so is the quotient $V^{\otimes n} / W^{\otimes n}$ for any integer $n \geq 2$.

Proof. The quotient $V^{\otimes n} / W^{\otimes n}$ admits a filtration with associated graded quotients of the form $W^{\otimes(n-l)} \otimes(V / W)^{\otimes l}, 1 \leq l \leq n$, and the product of a free $k[\mathbb{Z} / p \mathbb{Z}]$-module with an arbitrary one is free.

Lemma 2.9. For any associative unital algebra $A$ over $k$ such that the category of A-bimodules has finite homological dimension, and for any integer $n \geq 1$, the objects $\pi^{*} A_{\sharp}, i^{*} A_{\sharp} \in \operatorname{Fun}\left(\Lambda_{n}, k\right)$ are small in the sense of Definition 1.18. 
Proof. Assume first that $n=1$. Then we have

$$
j^{*} A_{\sharp}=C .(A) \otimes_{A^{o} \otimes A} A,
$$

where $C$. $(A)$ is the standard bar resolution of the diagonal $A$-bimodule $A$, and it suffices to prove that $C .(A)$ is effectively finite as a simplicial $A$-bimodule. Indeed, for any $m \geq 0$ the truncation $L^{\cdot} \tau_{!} F^{m} C$. $(A)$ is concentrated in degree $m$; since the category of $A$-bimodules has finite homological dimension, the space of maps from $L^{\cdot} \tau_{!} F^{m} C_{.}$(A) to $L^{\cdot} \tau_{!} F^{m+l} C_{.}(A)$ in $\mathcal{D}^{b}(A$-bimod) is trivial for sufficiently large $l$.

Thus $A_{\sharp}$ is indeed small. By (1.14), this immediately implies that $\pi^{*} A_{\sharp}$ is small for any $n$-indeed, the pullback with respect to the projection $\Delta^{o} \times \mathrm{pt}_{n} \rightarrow \Delta^{o}$ is obviously compatible with the stupid filtration.

Consider now $i^{*} A_{\sharp} \in \operatorname{Fun}\left(\Lambda_{p}, k\right)$. One checks easily that we have

$$
j_{n}^{*} i^{*} A_{\sharp}=C .(A)^{\otimes n} \otimes\left(A^{o} \otimes A\right) \otimes n A_{\sigma}^{\otimes n},
$$

where $A_{\sigma}^{\otimes n}$ is the diagonal $A^{\otimes n}$-bimodule $A^{\otimes n}$ with the bimodule structure twisted by the cyclic permutation $\sigma: A^{\otimes n} \rightarrow A^{\otimes n}$ - we let

$$
a \cdot m \cdot a^{\prime}=\operatorname{am\sigma }\left(a^{\prime}\right)
$$

for any $a, a^{\prime} \in A^{\otimes n}, m \in A_{\sigma}^{\otimes n}$. Moreover, the isomorphism (2.4) is compatible with the action of $\mathbb{Z} / n \mathbb{Z}$. Thus it suffices to prove that $C .(A)^{\otimes n}$ is effectively finite as a simplicial $\mathbb{Z} / n \mathbb{Z}$-equivariant $A$-bimodule. This immediately follows from Corollary 1.5 (ii).

Proposition 2.10. Assume given an associative unital algebra $A$ over $k$ equiped with a quasi-Frobenius map $F: A^{(1)} \rightarrow A^{\otimes p}$, and assume that the category of A-bidomules has finite homological dimension. Then there exists an isomorphism

$$
H_{.}\left(A^{(1)}\right)((u)) \cong H P .(A) .
$$

Proof. The map $F$, being compatible with the algebra structure, induces a map $F_{\sharp}: \chi^{*} A_{\sharp}^{(1)} \rightarrow\left(A^{\otimes p}\right)_{\sharp}$ of objects in $\operatorname{Fun}\left(\mathrm{B}_{p}, k\right)$ (explicitly, $F_{\sharp}([n])=F^{\otimes n}$ : $\left.A^{(1) \otimes n} \rightarrow A^{\otimes p n}\right)$. Restricting to $\Lambda_{p} \subset \mathrm{B}_{p}$ and using the isomorphism $\lambda^{*}\left(A^{\otimes p}\right)_{\sharp} \cong$ $i^{*} A_{\sharp}$, we obtain a map

$$
\widetilde{F}=\lambda^{*}\left(F_{\sharp}\right): \pi^{*} A_{\sharp}^{(1)} \rightarrow i^{*} A_{\sharp} .
$$


Since $F$ is injective, after evaluating at $[n] \in \Lambda_{p}$, this map gives an injective map

$$
F^{\otimes n}:\left(A^{(1)}\right)^{\otimes n} \rightarrow A^{\otimes p n},
$$

whose cokernel is a free $k[\mathbb{Z} / p \mathbb{Z}]$-module by Lemma 2.8. Therefore the map $\widetilde{F}$ is injective, and the quotient $i^{*} A_{\sharp} / \widetilde{F}\left(\pi^{*} A_{\sharp}^{(1)}\right)$ satisfies the equivalent conditions of Lemma 1.15 for the bifibration $\pi: \Lambda_{p} \rightarrow \Lambda$. Moreover, by Lemma 2.9 both $\pi^{*} A_{\sharp}$ and $i^{*} A_{\sharp}$ are small; then by Lemma 1.3 (i), so the quotient $i^{*} A_{\sharp} / \widetilde{F}\left(\pi^{*} A_{\sharp}^{(1)}\right)$. Therefore by Lemma 1.19, the induced map

$$
\widetilde{F}: H P .\left(\pi^{*} A_{\sharp}^{(1)}\right) \rightarrow H P .\left(i^{*} A_{\sharp}\right)
$$

is an isomorphism. Applying Lemma 1.14 and Lemma 1.16, we rewrite it as an isomorphism

$$
\widetilde{F}: H_{\bullet}\left(A^{(1)}\right)((u)) \cong H P .(A),
$$

which is our desired inverse Cartier map.

\section{Algebraic topology.}

In order to be able to construct a quasi-Frobenius map for any associative algebra $A$ over our fixed perfect field $k$ of characteristic char $k=p>0$, we have to modify Definition 2.4 in two ways:

(i) The map $F: A^{(1)} \rightarrow A^{\otimes p}$ has to be understood as a map of EilenbergMacLane spectra, not as a map of vector spaces.

(ii) The right-hand side $A^{\otimes p}$ has to be changed (in a way which does not, however, affect the Tate homology $\left.\check{H}^{\bullet}\left(\mathbb{Z} / p \mathbb{Z}, A^{\otimes p}\right)\right)$.

Here (ii) is not very drastic, we will return to it in Section 4. However, (i) has to be explained in detail. We start with the necessary preliminaries on spectra and the stable homotopy category.

3.1. Generalities on stable homotopy. While morally all of the material in this Section and in Section 4 comes from algebraic topology, formally our approach does not depend on topological notions. In this subsection, we describe the underlying topology. Logically, it is not necessary for the rest of the paper, so that a reader who is not interested in topology at all may skip this subsection (at 
a cost of having from now on to accept on faith the usefulness of some of the constructions). Because of its formal redundancy, and to save space, this subsection has been left rather loose and imprecise; in many cases, the reader would have to consult the original papers for exact mathematical statements. No material here is new, and most of it is quite standard. We mostly follow [FPSVW].

Recall that the derived category $\mathcal{D}(k)$ of $k$-vector spaces admits a natural triangulated embedding into the triangulated category of spectra, also known as stable homotopy category, which we denote by StHom (for its definition and basic properties, we refer the reader to any textbook on algebraic topology, such as, for instance, [A1]). The embedding, which we denote by $E M: \mathcal{D}(k) \rightarrow$ StHom, sends a complex of vector spaces to the corresponding Eilenberg-MacLane spectrum. We have a left-adjoint triangulated functor $H(k):$ StHom $\rightarrow \mathcal{D}(k)$, homology of a spectrum with coefficients in $k$. Thus for any $A \in \mathcal{D}(k), B \in \mathrm{StHom}$, we have

$$
\operatorname{Hom}(H(k)(B), A) \cong \operatorname{Hom}(B, E M(A)) .
$$

Recall that the stable homotopy category StHom has a natural tensor structure, the smash-product $-\wedge-$. The homology functor $H(k)$ is a tensor functor - we have a natural isomorphism $H(k)(A \wedge B) \cong H(k)(A) \otimes H(k)(B)$. The adjoint functor $E M$ is not tensor; we still have a natural "augmentation" map $E M(A) \wedge$ $E M(B) \rightarrow E M(A \otimes B)$, but it is not an isomorphism. The situation is analogous to the pair $\tau^{*}, \tau_{*}$ of the inverse and direct image of coherent sheaves with respect to a closed embedding $\tau: X \rightarrow Y$ of algebraic varieties; $H(k)$ plays the role of $\tau^{*}$, and $E M$ plays the role of $\tau_{*}$. The analogy extends quite far - for instance, we have a projection formula, which in this case reads as $E M(H(k)(A)) \cong A \wedge E M(k)$ (and can in fact be used as a definition of the functor $H(k)$ ).

Using the augmentation map $E M(A) \wedge E M(B) \rightarrow E M(A \otimes B)$, one checks easily that for an associative algebra $A$ in $\mathcal{D}(k)$, the spectrum $\operatorname{EM}(A)$ is an associative algebra in StHom. It is well-known that the notion of an associative algebra in a derived category is too weak - to get a useful notion, one has to lift the algebra structure to the level of models, and consider associative algebras in the category of complexes of vector spaces (in other words, differential graded algebras over $k$ ). The corresponding notion for StHom is that of a ring spectrum. For a long time, defining a ring spectrum had been a delicate task, since one didn't have a category of models for spectra equipped with a symmetric and associative tensor product (the reader can see this difficulty, for instance, in [A1, III.9]). 
This has been largely settled in the last ten years, with the appearance of several suitable model categories - see e.g. [EKMM], [HSS]. In fact, these days the term "brave new algebra" is commonly used, to indicate that rings in the usual sense should be replaced with "brave new rings", that is, rings in a suitable model category for spectra. To an algebraic geometer, this certainly seems brave, since while the multiplication in brave new rings is associative and/or commutative per requirements, the addition most certainly is not. Nevertheless, most of the homological constructions known for algebras and DG algebras now have their natural "topological" counterparts for ring spectra.

In the case of Hochschild homology $H H_{\text {. }}(A)$, the topological version has been known for a long time - it has been introduced by M. Bökstedt, [B]. To any ring spectrum $A$, one associates its topological Hochschild homology spectrum $T H H$. $(A)$, essentially by taking the usual Hochschild complex based on the bar resolution, and replacing tensor powers $A^{\otimes n}$ with smash powers $A \wedge A \wedge \cdots \wedge A$ (this is a modern formulation - since good model categories for spectra were not available at the time of [B], Bökstedt's original definition had to be more delicate).

When $A$ is an associative algebra over $k$ in the usual sense, one shows that in either of the models, $\operatorname{EM}(A)$ has a natural structure of a "brave new ring"; by abuse of notation, we denote $T H H_{\text {. }}(A)=T H H_{\text {. }}(E M(A))$. It is known that for an algebra over a field, $T H H .(A)$ is an Eilenberg-MacLane spectrum, too. However, since the Eilenberg-Maclane functor $E M$ is not tensor, $T H H_{\text {. }}(A)$ is not isomorphic to $\operatorname{EM}\left(H H_{\bullet}(A)\right)$ - in fact, it is much larger.

It turns out that it is $T H H .(A)$ which is the natural domain of definition of the inverse Cartier map for a general associative unital algebra $A$ over $k$; the map that we will construct in Section 4 is a map of the form

$$
T H H_{.}(A)((u)) \stackrel{C^{-1}}{\longrightarrow} E M(H P .(A)) .
$$

Since $T H H_{\text {. }}(A)$ is much larger than $H H_{\text {. }}(A)$, this map $C^{-1}$ is no longer an isomorphism (nor does it factor through the natural augmentation map $T H H .(A) \rightarrow$ $\left.\operatorname{EM}\left(H_{\bullet}(A)\right)\right)$.

As we have mentioned in the Introduction, a version of such a generalized Cartier map can be obtained within the framework of Topological Cyclic Homology, as a part of the cyclotomic spectrum package ([HM], $[\mathrm{BHM}])$. And in fact, 
topology gives much more. Not only the algebra $A^{(1)}$ in Definition 2.4 can be treated as ring spectrum, but also the algebra $A^{\otimes p}$, - so that in (3.1), the righthand side is refined to a version of the periodic cyclic homology for ring spectra (which is much larger than $\operatorname{EM}(H P .(A))$ ). However, this really requires topological methods, already in the definition of topological cyclic homology spectrum $T H C$. $(A)$. For our purposes, a cruder Cartier map of the form (3.1) is enough, which only involves $T H H_{\text {. }}(A)$. As it turns out, one can actually work with THH. (A) without using spectra at all.

To understand how to do this, and to see better the difference between $T H H$. $(A)$ and $H H_{.}(A)$, we consider the composition St $=H(k) \circ E M: \mathcal{D}(k) \rightarrow \mathcal{D}(k)$. For any $k$-vector space $V$, there is a non-canonical quasiisomorphism $\operatorname{St}(V) \cong$ $V \otimes \operatorname{St}(k)$, so that $\operatorname{St}(V)$ is additive in $V$ in a certain sense. The homology of the complex $\operatorname{St}(k)$ is identified, more or less by definition, with the dual to the Steenrod algebra.

For any $A, B \in \mathcal{D}(k)$ we have a natural map $\mathrm{St}(A) \otimes \mathrm{St}(B) \rightarrow \mathrm{St}(A \otimes B)$, and for any associative $k$-algebra $A, \operatorname{St}(A)$ is an associative algebra in $\mathcal{D}(k)$ (if $A=k$, this corresponds to the coalgebra structure on the Steenrod algebra $\left.\operatorname{St}(k)^{*}\right)$. Since the functor $H(k)$ is a tensor functor, we see, almost by definition, that

$$
H(k)\left(T H H_{.}(A)\right) \cong H_{.}(\mathrm{St}(A)) .
$$

By adjunction, constructing a map as in (3.1) is equivalent to constructing a map $H(k)\left(T H H_{\bullet}(A)\right)((u)) \rightarrow H P .(A)$, and this can be rewritten as

$$
H H_{.}(\mathrm{St}(A))((u)) \rightarrow H P .(A),
$$

which is a map in $\mathcal{D}(k)$. While $\operatorname{St}(A) \cong A \otimes \operatorname{St}(k)$ is much larger than $A$, so that $H H_{\text {. }}(\mathrm{St}(A))$ is larger than $H H_{\text {. }}(A)$, it is nevertheless defined on the level of $\mathcal{D}(k)$, without any need to go to StHom.

This is the approach that we want to use, - except that it does not work as stated. The problem is, the right-hand side of (3.2) is not well-defined; the structure of an algebra in $\mathcal{D}(k)$ on $\operatorname{St}(A)$ is too weak for that. We need a DG algebra realization of $\operatorname{St}(A)$, or some replacement for it.

It is rather easy to realize $\operatorname{St}(A)$ as a complex of $k$-vector spaces, and more generally, to lift the functor St $: \mathcal{D}(k) \rightarrow \mathcal{D}(k)$ to the level of complexes - at least if we restrict ourselves to the complexes which are trivial in positive degrees. 
Namely, we can use the Dold-Kan equivalence of Subsection 1.2 between the category $C_{\leq 0}(k)$ of such complexes and the category $\operatorname{Fun}\left(\Delta^{o}, k\right)$ of simplicial $k$ vector spaces. By abuse of notation, we will not distinguish between the two; in particular, we will use the notation $V$. $[l]$ for the downward shift by $l$ when $V$. is given not as a complex but as a simplicial vector space. Dold showed that for any vector space $V$, the simplicial vector space $V[l] \in \operatorname{Fun}\left(\Delta^{o}, k\right)$, considered as a simplicial set, represents the homotopy type of the Eilenberg-MacLane space $K(V, l)$. For any pointed simplicial set $\left\langle X_{\text {. }}\right.$ pt $\rangle$, we denote by $\overline{k[X .]} \in \operatorname{Fun}\left(\Delta^{o}, k\right)$ its reduced $k$-linear span - that is, we set

$$
\overline{k\left[X_{.}\right]_{i}}=k\left[X_{i}\right] / k \cdot \mathrm{pt}
$$

for any index $i$. The simplicial set $V[l]$ is pointed, with 0 being the distinguished point; the complex $\overline{k[V[l]]}$ computes the reduced homology of the space $K(V, l)$. For any $l$, the transgression map relating $H_{\bullet}(K(V, l))$ and $H_{\bullet}(K(V, l+1))$ can be lifted to a canonical map

$$
\tau: \overline{k[V[l]]}[1] \rightarrow \overline{k[V[l+1]]}
$$

These maps are sufficiently canonical so that they are equally well defined when $V$ is replaced by a complex $V . \in \operatorname{Fun}\left(\Delta^{o}, k\right)$. Moreover, the complex $\overline{k\left[V_{\bullet}[l]\right]}$ is trivial in homological degrees $<l$, therefore the maps $\tau$ can be shifted upwards to form an injective system. We set

$$
\text { St. }\left(V_{\bullet}\right)=\lim _{\underset{\tau}{\rightarrow}} \overline{k\left[V_{\bullet}[l]\right]}[-l] .
$$

This is a (non-additive) functor from $\operatorname{Fun}\left(\Delta^{o}, k\right)$ to itself, and although it is not additive, it is homotopy-invariant - that is, St. sends quasiisomorphisms to quasiisomorphisms, and therefore descends to an endofunctor of the category $D_{\leq 0}(k)$. Stabilizing it with respect to the shifts, we get the functor $\mathrm{St}=H(K) \circ$ $E M: \mathcal{D}(k) \rightarrow \mathcal{D}(k)$.

Unfortunately, this simplicial model St. for the functor St is not compatible with the tensor products - one cannot construct a functorial and associative map St. $(V) \otimes \mathrm{St}_{\text {. }}(W) \rightarrow \mathrm{St} .(V \otimes W)$ (at least, not without much effort, many noncanonical choices, and horrible combinatorics). There are two ways to overcome this difficulty.

Segal's approach. One can use a general principle that first appeared in G. Segal's approach to infinite loop spaces ([Se], $[\mathrm{A} 2, \S 2.5])$. Namely, in order to define 
$H H$. $(\mathrm{St}(A))$, we do not need an associative $\mathrm{DG}$ algebra structure on $\operatorname{St}(A)$ on the nose - what we need is a collection of complexes $\mathrm{St}^{n}(A)$ representing tensor powers $\operatorname{St}(A)^{\otimes n}$, and all the maps between them that would come from an associative algebra structure, but the complex $\operatorname{St}^{n}(A)$ does not have to be actually isomorphic to $\mathrm{St}^{1}(A)^{\otimes n}$ - it is sufficient to assume that these two are quasiisomorphic. In fact, it was this principle that was used in the original definition of the topological Hochschild homology in [B], which predates the modern strictly symmetric and associative monoidal model categories for spectra.

The cube construction. In spite of all of the above, there does exist a sufficiently direct and functorial way to associate a complex $Q .(V)$ to a vector space $V$ so that $Q .(V)$ represents $\mathrm{St}(V)$, and we have functorial and associative product maps $Q .(V) \otimes Q .(W) \rightarrow Q .(V \otimes W)$. This has been introduced by S. Eilenberg and S. MacLane in $[\mathrm{EM}]$, at the dawn of homological algebra, under the name of the cube or the $Q$-construction. The product is known as Dixmier product. The cube construction lingered in a relative obscurity for a long time (with some exceptions, e.g. [Br]). As far as I know, it had been resurrected by M. Jibladze and T. Pirashvili in [JP], and in any case, it has been used extensively by T. Pirashvili ever since, in a formidable body of work. In particular, one can develop a version of the Hochschild homology based on the cube construction; this has indeed been done by S. MacLane [Mc], and it is nowadays known as MacLane homology. And for an algebra $A$ over a field $k$, it has been proved in [PW], [FPSVW] that the MacLane homology $H M_{\text {. }}(A)$, considered as an Eilenberg-MacLane spectrum, coincides with the topological Hochschild homology $T H H_{\text {. }}(A)$ (this is probably the simplest way to prove that $T H H .(A)$ is an Eilenberg-MacLane spectrum). For our applications, we only need to know $H(k)(T H H .(A))$; this is even easier to describe using the cube construction, since it coincides precisely with $H_{\bullet}(Q .(A))$.

For the convenience of the reader, we will spend some time and describe both approaches (not the last reason for this being that, while the cube construction can be presented directly, it looks rather ad hoc and incomprehensible without a more conceptual explanation). We will mostly follow the exposition given by J.-L. Loday and T. Pirashvili in [LP], with some additional modifications which, we hope, further clarify and simplify the picture. 
3.2. Additivization. We sum up briefly the relevant facts from the last subsection, for the benefit of a reader who skipped it. Recall the Dold-Kan equivalence of Subsection 1.2 between the category $\operatorname{Fun}\left(\Delta^{o}, k\right)$ of simplicial $k$-vector spaces and the category $C_{\leq 0}(k)$ of complexes of $k$-vector spaces which are trivial in positive degrees. For any $l \geq 0$ and any vector space $V$, the simplicial vector space corresponding to the complex $V[l]$, considered as a simplicial set, represents the homotopy type of the Eilenberg-Maclane space $K(V, l)$. We have transgression maps of simplicial $k$-vector spaces

$$
\tau: \overline{k[V[l]]} \rightarrow \overline{k[V[l+1]]}[-1],
$$

where $\overline{k[S]}=k[S] / k \cdot \mathrm{pt}$ is the reduced $k$-linear span of a pointed set $\langle S, \mathrm{pt}\rangle$, and $V[l]$ is considered as a pointed set by taking 0 as the distinguished point. Passing to the limit, we get the complex

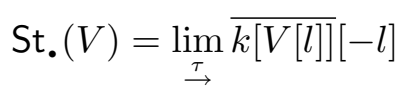

which computes the homology of the Eilenberg-MacLane spectrum corresponding to $V$ (sometimes called the stable homology of the commutative group $V$ ).

The complex St. $(V)$ is very large. In this subsection, we describe a much more efficient way to represent the stable homology of a vector space, based on the work of T. Pirashvili (see [LP] and references therein).

The idea is very natural, from a certain point of view. It is known that the homology of the Eilenberg-MacLane spaces $K(V, l)$ is additive "in stable range of degrees": for two $k$-vector spaces $V_{1}, V_{2}$, we have $H_{i}\left(K\left(V_{1}, l\right)\right) \oplus H_{i}\left(K\left(V_{2}, l\right)\right) \cong$ $H_{i}\left(K\left(V_{1} \oplus V_{2}, l\right)\right)$ whenever $0<i<2 l$. In the limit, St. $\left(V_{1}\right) \oplus \operatorname{St} .\left(V_{2}\right)$ is naturally quasiisomorphic to $\operatorname{St}\left(V_{1} \oplus V_{2}\right)$. Thus to cut the complexes $k[V[l]]$ down to size, the natural thing to do is to take "the additive part" of the functor $V \mapsto k[V[l]]$. Somewhat suprisingly, if the additivization procedure is done in a sufficiently functorial way, passing to the limit with respect to the transgression maps becomes unnecessary - the additive part of the functor $k[V[l]]$ does not depend on $l$. Therefore it suffices to compute it for $l=0$, and this can be done in a reasonably explicit way.

To develop the addivization machinery systematically, we fix a functor $F$ from the category $k$-Vect of $k$-vector spaces to itself. We do not assume $F$ to be 
additive; it is reasonable to assume though, that $F$ is finitely presented (that is, commutes with filtered direct limits).

For any vector space $V$, we have three natural maps $d_{1}, d_{2}, d_{12}: V \rightarrow V \oplus V$, given by $d_{1}(v)=v \oplus 0, d_{2}(v)=0 \oplus v, d_{12}(v)=v \oplus v$. Applying the functor $F$, we get three maps from $F(V)$ to $F(V \oplus V)$. We say that an element $v \in F(V)$ is primitive if $F\left(d_{12}\right)(v)=F\left(d_{1}\right)(v)+F\left(d_{2}\right)(v)$. It is easy to check that $F$ is additive if and only if $F\left(d_{12}\right)=F\left(d_{1}\right)+F\left(d_{2}\right)$ for any $V$, so that every element in $F(V)$ is primitive. For a general $F$, we define $F^{\prime}(V) \subset F(V)$ as the subspace of all primitive elements. This fits into a short exact sequence

$$
0 \longrightarrow F^{\prime}(V) \longrightarrow F(V) \stackrel{F\left(d_{1}\right)+F\left(d_{2}\right)-F\left(d_{12}\right)}{\longrightarrow} F(V \oplus V),
$$

and defines an additive functor $F^{\prime}: k$-Vect $\rightarrow k$-Vect, the maximal additive subfuntor of our fixed functor $F$.

Example 3.1. If $F(V)=V^{\otimes l}$, the $l$-th tensor power functor for some integer $l \geq 2$, then $F^{\prime}=0$. The same is true for the constant functor $F(V)=W, W$ a fixed $k$-vector space. On the other hand, if $F(V)=k\left(V^{*}\right)$, the space of all $k$-valued functions on the dual $k$-vector space $V^{*}$, then a function $f: V^{*} \rightarrow k$ is primitive as an element of $F(V)$ if and only if it is linear, so that for a finitedimensional $V$, we have $F^{\prime}(V)=V \otimes_{\mathbb{Z}} k$.

To obtain a sufficiently nice additivization procedure, we have to define "higher derived functors" of the correspondence $F \mapsto F^{\prime}$, so that (3.4) is extended, in a certain way, to a long exact sequence. To do this, we need to consider not only the sum $V \oplus V$, but all the sums $V^{\oplus n}, n \geq 1$, and natural maps between them. We note right away that for technical reasons, we prefer to work with homology of Eilenberg-MacLane spaces, not their cohomology; therefore we will take the dual point of view and study the maximal additive quotient of a functor $F: k$-Vect $\rightarrow k$-Vect rather than its maximal additive subfunctor $F^{\prime} \subset F$.

Denote by $\Gamma_{+}$the category of finite pointed sets (that is, sets with a distinguished element). Denote by $[n]_{+} \in \Gamma_{+}$the set with $(n+1)$ elements, one of them distinguished - in other words, we have $[n]_{+}=\langle[n] \amalg\{\mathrm{pt}\}$, pt $\rangle$, where $[n]$ is the set with $n$ elements, and pt is the fixed point. For any set $A$ and subset $B \subset A$, we, as usual, denote by $A / B$ the pointed set obtained by gluing together all the elements in $B$ to obtain the fixed point pt $\in A / B$. For any two pointed 
sets $\left[n_{1}\right]_{+},\left[n_{2}\right]_{+}$, we have their reduced sum and their smash product,

$$
\begin{aligned}
& {\left[n_{1}\right]_{+} \vee\left[n_{2}\right]_{+}=\left(\left[n_{1}\right]_{+} \amalg\left[n_{2}\right]_{+}\right) /(\{\mathrm{pt}\} \coprod\{\mathrm{pt}\}),} \\
& {\left[n_{1}\right]_{+} \wedge\left[n_{2}\right]_{+}=\left(\left[n_{1}\right]_{+} \times\left[n_{2}\right]_{+}\right) /\left(\left(\left[n_{1}\right]_{+} \times \mathrm{pt}\right) \cup\left(\mathrm{pt} \times\left[n_{2}\right]_{+}\right)\right) .}
\end{aligned}
$$

We obviously have $\left[n_{1}\right]_{+} \vee\left[n_{2}\right]_{+} \cong\left[n_{1}+n_{2}\right]_{+}$and $\left[n_{1}\right]_{+} \wedge\left[n_{2}\right]_{+} \cong\left[n_{1} n_{2}\right]_{+}$.

Consider the functor category $\operatorname{Fun}\left(\Gamma_{+}^{o}, k\right)$. For any finite pointed set $[n]_{+} \in \Gamma_{+}$, let $T_{n} \in \operatorname{Fun}\left(\Gamma_{+}^{o}, k\right)$ be the functor corepresented by $[n]_{+} \in \Gamma_{+}^{o}-$ that is, the functor given by

$$
T_{n}\left([m]_{+}\right)=k\left[\Gamma_{+}\left([n]_{+},[m]_{+}\right)\right]^{*} .
$$

We obviously have $T_{n} \cong T_{1}^{\otimes n}$, and $T_{0}$ is the constant functor $k \in \operatorname{Fun}\left(\Gamma_{+}^{o}, k\right)$, $T_{0}\left([m]_{+}\right)=k$ for any $[m]_{+} \in \Gamma_{+}$. The $k$-vector space $T_{1}\left([m]_{+}\right)$is the $k$-linear span of the finite set $[m]_{+}$. Being corepresentable, the functors $T_{n}$ are injective, and they give a set of injective generators of the category $\operatorname{Fun}\left(\Gamma_{+}^{o}, k\right)$. To get a more economical set, split $T_{1}=T \oplus T_{0}$, where $T$ is the reduced $k$-linear span functor - that is, we have

$$
T\left([n]_{+}\right)={\overline{k\left[[n]_{+}\right.}}^{*}
$$

for any finite pointed set $[n]_{+} \in \Gamma_{+}$. For every $n$, the tensor power $T^{\otimes n} \in$ $\operatorname{Fun}\left(\Gamma_{+}, k\right)$ is a direct summand of $T_{n}=T_{1}^{\otimes n}$, thus injective; since $T_{n}=T_{1}^{\otimes n}$ splits into a sum of summands of the form $T^{\otimes l} \otimes T_{0}^{\otimes n-l} \cong T^{\otimes l}, l \leq n$, the functors $T^{\otimes n}$ also form a set of injective generators of $\operatorname{Fun}\left(\Gamma_{+}^{o}, k\right)$.

Lemma 3.2. For any $n, m \geq 1$, the $k$-vector space $\operatorname{Hom}\left(T^{\otimes n}, T^{\otimes m}\right)$ can be naturally identified with the $k$-linear span of the set $\operatorname{Epi}([m],[n])$ of all surjective maps from the set $[m]$ with $m$ elements to the set $[n]$ with $n$ elements. In particular, $\operatorname{Hom}\left(T^{\otimes n}, T^{\otimes m}\right)=0$ if $n>m$.

Proof. Since $T_{m}$ and $T_{n}$ are corepresentable, we have

$$
\operatorname{Hom}\left(T_{m}, T_{n}\right)=k\left[\Gamma_{+}\left([m]_{+},[n]_{+}\right)\right] .
$$

We will say that a map $f \in \Gamma_{+}\left([m]_{+},[n]_{+}\right)$is non-degenerate if the preimage of the fixed point in $[n]_{+}$is exactly the fixed point in $[m]_{+}$, and we will say that a map $f \in \Gamma_{+}\left([m]_{+},[n]_{+}\right)$is a degenerate surjection if it is bijective over the complement to the fixed point in $[n]_{+}$. The set of non-degenerate surjective maps from $[n]_{+}$to $[m]_{+}$obviously coincides with the set Epi $([n],[m])$ of surjective 
maps from $[n]=[n]_{+} \backslash\{\mathrm{pt}\}$ to $[m]=[m]_{+} \backslash\{\mathrm{pt}\}$. A degenerate surjection is leftinverse to a unique injective map. Every map $f$ obviously uniquely factors as $f=e(f) \circ \bar{f} \circ p(f)$, where $p(f)$ is a degenerate surjection, $\bar{f}$ is a non-degenerate surjection, and $e(f)$ is injective. This gives a decomposition

$$
\Gamma_{+}\left([m]_{+},[n]_{+}\right)=\coprod_{S \subset[n], S^{\prime} \subset[m]} \operatorname{Epi}\left(S^{\prime}, S\right),
$$

where for any $f \in \Gamma_{+}\left([m]_{+},[n]_{+}\right), S \subset[n]$ is the image of the injection $e(f)$ with the fixed point pt removed, and $S^{\prime} \in[\mathrm{m}]$ is the image of the injection left-inverse to $p(f)$ with the fixed point removed.

On the other hand, for any $l, T_{l}=T_{1}^{\otimes l}=\left(T \oplus T_{0}\right)^{\otimes l}=(T \oplus k)^{\otimes l}$ decomposes as

$$
T_{l}=\bigoplus_{S \subset[l]} T^{\otimes|S|}
$$

where $S \subset[l]$ are all subsets, and $|S|$ is the cardinality of the set $|S|$. Thus we have

$$
\operatorname{Hom}\left(T_{n}, T_{m}\right)=\bigoplus_{S \subset S_{n}, S^{\prime} \subset S_{m}} \operatorname{Hom}\left(T^{\otimes|S|}, T^{\otimes\left|S^{\prime}\right|}\right),
$$

and one checks easily that under the correspondence (3.7), an injection gives a map that vanishes on the summand $T^{\otimes n} \subset T_{n}$, and a degenerate surjection gives a map whose image is complementary to the summand $T^{\otimes m} \subset T_{m}$. Comparing (3.9) and (3.8) with (3.7), and using induction on $n$ and $m$, we get the claim.

Now, consider the category $\operatorname{Fun}\left(\Gamma_{+}, k\right)$. Dualizing (3.6), we define a functor $T^{*} \in \operatorname{Fun}\left(\Gamma_{+}, k\right)$ by

$$
T^{*}\left([n]_{+}\right)=T\left([n]_{+}\right)^{*}=\overline{k\left[[n]_{+}\right]} .
$$

We note that by duality, the tensor powers $T^{* \otimes n}, n \geq 0$ form a set of projective generators of $\operatorname{Fun}\left(\Gamma_{+}, k\right)$. By Lemma 3.2 we have $\operatorname{Hom}\left(T^{*}, T^{*}\right)=k$, so that the correspondence

$$
W \mapsto W \otimes T^{*}
$$

gives a full embedding $k$-Vect $\rightarrow \operatorname{Fun}\left(\Gamma_{+}, k\right)$. Let us say that a functor $E \in$ $\operatorname{Fun}\left(\Gamma_{+}, k\right)$ is linear if it lies in the image of this full embedding. The embedding admits an adjoint given by $E \mapsto E \otimes_{\Gamma_{+}} T ;\left(E \otimes_{\Gamma_{+}} T\right) \otimes T^{*}$ is the maximal linear quotient of a functor $E \in \operatorname{Fun}\left(\Gamma_{+}, k\right)$. 
Now, starting from our fixed functor $F: k$-Vect $\rightarrow k$-Vect, we can form a functor $F_{\natural}: k$-Vect $\rightarrow \operatorname{Fun}\left(\Gamma_{+}, k\right)$ by setting

$$
F_{\natural}(V)\left([n]_{+}\right)=F\left(V \otimes T^{*}\left([n]_{+}\right)\right)=F\left(V^{\oplus n}\right)
$$

for any $k$-vector space $V$. If the functor $F$ is additive, we have $F_{\natural}\left([n]_{+}\right)=$ $F\left(V^{\oplus n}\right)=F(V)^{\oplus n}=F(V) \otimes T^{*}\left([n]_{+}\right)$, so that $F_{\sharp}(V) \in \operatorname{Fun}\left(\Gamma_{+}, k\right)$ is linear. In general, we can try to extract the maximal additive quotient of the functor $F$ by taking the maximal linear quotient of the functor $F_{\natural}(V)$. It turns out that this works, and moreover, it gives the correct "higher derived functors" of the additivization procedure.

Definition 3.3. The additivization Add. $(F)$ of the functor $F$ is the functor from $k$-Vect to $\mathcal{D}^{-}(k)$ defined by

$$
\text { Add. }(F)(V)=F_{\natural}(V) \stackrel{\llcorner}{\otimes} \Gamma_{+} T,
$$

where $\stackrel{\llcorner}{\otimes} \Gamma_{+}$is the derived functor of the tensor product functor $\otimes_{\Gamma_{+}}$.

Lemma 3.4. For any $k$-vector spaces $V_{1}, V_{2}$, the map

$$
\text { Add. }(F)\left(V_{1}\right) \oplus \operatorname{Add} .(F)\left(V_{2}\right) \stackrel{\text { Add. }(F)\left(i_{1}\right) \oplus \text { Add. }(F)\left(i_{2}\right)}{\longrightarrow} \text { Add. }(F)\left(V_{1} \oplus V_{2}\right)
$$

induced by the natural embeddings $i_{1}: V_{1} \rightarrow V_{1} \oplus V_{2}, i_{2}: V_{2} \rightarrow V_{1} \oplus V_{2}$ is a quasiisomorphism.

Proof. Define $F_{\natural}\left(V_{1}, V_{2}\right) \in \operatorname{Fun}\left(\Gamma_{+} \times \Gamma_{+}, k\right)$ by

$$
\begin{aligned}
F_{\natural}\left(V_{1}, V_{2}\right)\left(\left[n_{1}\right]_{+} \times\left[n_{2}\right]_{+}\right) & =F\left(\left(V_{1} \otimes T^{*}\left(\left[n_{1}\right]_{+}\right)\right) \oplus\left(V_{2} \otimes T^{*}\left(\left[n_{2}\right]_{+}\right)\right)\right) \\
& =F\left(V_{1}^{\oplus n_{1}} \oplus V_{2}^{\oplus n_{2}}\right) .
\end{aligned}
$$

Then $o_{l}^{*} F_{\natural}\left(V_{1}, V_{2}\right) \cong F_{\natural}\left(V_{l}\right), l=1,2$, where $o_{1}, o_{2}$ are given by $o_{1}\left([n]_{+}\right)=[n]_{+} \times$ $[1]_{+}, o_{2}\left([n]_{+}\right)=[1]_{+} \times[n]_{+}$, and $\delta^{*} F_{\natural}\left(V_{1}, V_{2}\right) \cong F_{\natural}\left(V_{1} \oplus V_{2}\right)$, where $\delta: \Gamma_{+} \rightarrow$ $\Gamma_{+} \times \Gamma_{+}$is the diagonal embedding. By (1.4), this implies that

$$
\text { Add. } \begin{aligned}
(F)\left(V_{1} \oplus V_{2}\right) & =F_{\natural}\left(V_{1} \oplus V_{2}\right) \stackrel{\llcorner}{\otimes} \Gamma_{+} T \cong \delta^{*} F_{\natural}\left(V_{1}, V_{2}\right) \stackrel{\llcorner}{\otimes} \Gamma_{+} T \\
& \cong F_{\natural}\left(V_{1}, V_{2}\right) \stackrel{\llcorner}{\otimes_{\Gamma_{+}} \times \Gamma_{+}} L \cdot \delta_{!} T .
\end{aligned}
$$

But the diagonal embedding $\delta$ admits a left-adjoint $\Sigma: \Gamma_{+} \times \Gamma_{+} \rightarrow \Gamma_{+}$given by $\Sigma\left(\left[n_{1}\right]_{+} \times\left[n_{2}\right]_{+}\right)=\left[n_{1}\right]_{+} \vee\left[n_{2}\right]_{+}$, as in (3.5). Therefore $\delta_{!} \cong \Sigma^{*}$. In particular, $\delta_{!} \cong L \cdot \delta_{!}$is exact, and we have

$$
\text { Add. }(F)\left(V_{1} \oplus V_{2}\right) \cong F_{\natural}\left(V_{1}, V_{2}\right) \stackrel{\llcorner}{\otimes} \Gamma_{+} \times \Gamma_{+} \Sigma^{*} T .
$$


It remains to notice that $\Sigma^{*} T \cong p_{1}^{*} T \oplus p_{2}^{*} T$, where $p_{1}, p_{2}: \Gamma_{+} \times \Gamma_{+} \rightarrow \Gamma_{+}$are the two natural projections, and $p_{l}$ is left-adjoint to $o_{l}, l=1,2$, so that $p_{l}^{*} \cong L^{*} \sigma_{l !}$. Since $o_{l}^{*} F_{\natural}\left(V_{1}, V_{2}\right) \cong F_{\natural}\left(V_{l}\right), l=1,2$, we may again apply (1.4) and deduce

$$
\begin{aligned}
& F_{\text {七 }}\left(V_{1}, V_{2}\right) \stackrel{\llcorner}{\otimes} \Gamma_{+} \times \Gamma_{+} \Sigma^{*} T \cong F_{\text {七 }}\left(V_{1}, V_{2}\right) \stackrel{\llcorner}{\otimes} \Gamma_{+} \times \Gamma_{+}\left(p_{1}^{*} T \oplus p_{2}^{*} T\right) \\
& \cong\left(F_{\mathrm{\natural}}\left(V_{1}, V_{2}\right) \stackrel{\llcorner}{\otimes} \Gamma_{\Gamma_{+} \times \Gamma_{+}} L^{\cdot} \sigma_{1 !} T\right) \oplus\left(F_{\mathrm{\natural}}\left(V_{1}, V_{2}\right) \stackrel{\stackrel{\llcorner}{\otimes}}{\Gamma_{+} \times \Gamma_{+}} L^{\cdot} \sigma_{2 !} T\right) \\
& \cong\left(F_{\natural}\left(V_{1}\right) \stackrel{\llcorner}{\otimes} \Gamma_{+} T\right) \oplus\left(F_{\natural}\left(V_{2}\right) \stackrel{\llcorner}{\otimes} \Gamma_{+} T\right) \text {, }
\end{aligned}
$$

as required.

Thus Add. $(F)$ is indeed additive, as the name suggests. More generally, for any small category $C$, we can extend $F$ to a functor $F^{C}$ from $\operatorname{Fun}(C, k)$ to itself by applying it pointwise. Analogously, we can apply $F_{\natural}(V)$ pointwise; this gives a functor $F_{\natural}^{C}: \operatorname{Fun}(C, k) \rightarrow \operatorname{Fun}\left(\Gamma_{+} \times C, k\right)$. We define the relative additivization $\operatorname{Add}^{C}(F): \operatorname{Fun}(C, k) \rightarrow \mathcal{D}^{-}(C, k)$ by

$$
\operatorname{Add}_{.}^{C}(F)(E)=F_{\natural}^{C}(E) \stackrel{\llcorner}{\otimes} \Gamma_{+} T \in \mathcal{D}^{-}(C, k)
$$

for any $E \in \operatorname{Fun}(C, k)$.

In the case when $C=k$-Vect $\mathrm{fg}^{\mathrm{f}}$ is the category of finite-dimensional $k$-vector spaces, relative addivization with respect to $C$ allows one to refine the usual additivization Add. $(F): k$-Vect $\rightarrow \mathcal{D}(k)$ to an object in the triangulated category $\mathcal{D}^{-}\left(k\right.$-Vect $\left.{ }^{\mathrm{fg}}, k\right)$. Namely, we consider the tautological embedding $k$-Vect $\mathrm{fg}^{\mathrm{fg}} \rightarrow$ $k$-Vect as an object $\mathrm{Id} \in \operatorname{Fun}\left(k\right.$-Vect $\left.{ }^{\mathrm{fg}}, k\right)$, and we let

$$
\text { Add. }(F)=\operatorname{Add}^{k-\operatorname{Vect}}{ }^{\mathrm{fg}}(F)(\text { Id }) \in \mathcal{D}^{-}\left(k-\operatorname{Vect}^{\mathrm{fg}}, k\right) .
$$

This is consistent with previous notation - evaluating Add. $(F)$ at $V \in k$-Vect ${ }^{f g}$ gives Add. $(F)(V)$ as defined in Definition 3.3. Moreover, since Add. $(F)$ is obviously linear in $F$, it extends to a functor from $\mathcal{D}^{-}\left(k\right.$-Vect $\left.{ }^{\mathrm{fg}}, k\right)$ to itself, and we can state precisely in what sense $\operatorname{Add} .(F)$ is the "universal additivization" of a functor $F \in \mathcal{D}^{-}\left(k\right.$-Vect $\left.{ }^{\mathrm{fg}}, k\right)$.

Lemma 3.5. Let $\operatorname{Fun}^{\text {add }}\left(k-\mathrm{Vect}^{\mathrm{fg}}, k\right) \subset \operatorname{Fun}\left(k-\mathrm{Vect}^{\mathrm{fg}}, k\right)$ be the full subcategory of additive functors, and let $\mathcal{D}^{\text {add }}\left(k\right.$-Vect $\left.{ }^{\mathrm{fg}}, k\right) \subset \mathcal{D}^{-}\left(k\right.$-Vect $\left.{ }^{\mathrm{fg}}, k\right)$ be the full subcategory spanned by complexes with homology in $\mathrm{Fun}^{\text {add }}\left(k\right.$-Vect $\left.{ }^{\mathrm{fg}}, k\right)$. Then the functor Add. is left-adjoint to the embedding $\mathcal{D}^{\text {add }}\left(k\right.$-Vect $\left.{ }^{\mathrm{fg}}, k\right) \subset \mathcal{D}^{-}\left(k\right.$-Vect $\left.{ }^{\mathrm{fg}}, k\right)$. 
Proof. By Lemma 3.4, Add. $(F)$ lies in $\mathcal{D}^{\text {add }}\left(k\right.$-Vect $\left.{ }^{\mathrm{fg}}, k\right)$ for any $F$, so that Add. is indeed a functor from $\mathcal{D}^{-}\left(k\right.$-Vect $\left.{ }^{\mathrm{fg}}, k\right)$ to $\mathcal{D}^{\text {add }}\left(k\right.$-Vect $\left.{ }^{\mathrm{fg}}, k\right) \subset \mathcal{D}^{-}\left(k\right.$-Vect $\left.{ }^{\mathrm{fg}}, k\right)$. The adjunction map $F \rightarrow$ Add. $(F)$ is induces by the tautological map

$$
F(V)=F_{\natural}(V)\left([1]_{+}\right) \rightarrow F_{\natural}(V) \stackrel{\llcorner}{\otimes} \Gamma_{+} T .
$$

To finish the proof, it remains to prove that this adjunction map is an isomorphism for any $V \in k$-Vect ${ }^{\text {fg }}$ whenever $F$ is additive. Since for such $F$, the functor $F_{\natural}(V) \in \operatorname{Fun}\left(\Gamma_{+}, k\right)$ is linear, so that $F_{\natural}(V) \cong W \otimes T^{*}$ for some $k$-vector space $W$, this immediately follows from Lemma 3.2: $F_{\mathrm{\natural}}(V)$ is projective in $\operatorname{Fun}\left(\Gamma_{+}, k\right)$, and $F_{\natural}(V) \otimes_{\Gamma_{+}} T \cong W \cong F_{\natural}(V)\left([1]_{+}\right)$.

Remark 3.6. One can also visualize the additivization procedure in the following way. Treat $T^{*} \in \operatorname{Fun}\left(\Gamma_{+}, k\right)$ as a functor $\theta: \Gamma_{+} \rightarrow k$-Vect ${ }^{\mathrm{fg}}$. Then $E \in \operatorname{Fun}\left(k-\operatorname{Vect}{ }^{\mathrm{fg}}, k\right)$ is additive if and only if $\theta^{*} E \in \operatorname{Fun}\left(\Gamma_{+}, k\right)$ is linear, so that we have a Cartesian square

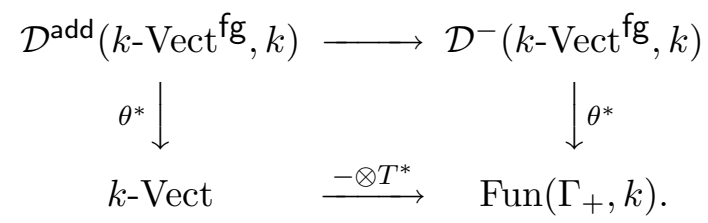

Lemma 3.5 is then a certain base-change-like property for this Cartesian square. We have no idea in what generality one should expect similar statements to hold; in particular, if one replaces $\Gamma_{+}$with the simplicial category $\Delta$, then the square remains Cartesian, but an analog of Lemma 3.5 is certainly false.

For a general small category $C$, we have the following refinement of Lemma 3.4.

Lemma 3.7. For an arbitrary small category $C$, and for any short exact sequence

$$
0 \longrightarrow E_{1} \longrightarrow E_{2} \longrightarrow E_{3} \longrightarrow 0
$$

in $\operatorname{Fun}(C, k)$, we have a natural distinguished triangle

$$
\operatorname{Add}^{C}(F)\left(E_{1}\right) \longrightarrow \operatorname{Add}^{C}(F)\left(E_{2}\right) \longrightarrow \operatorname{Add}^{C}(F)\left(E_{3}\right) \longrightarrow
$$

Proof. For any vector space $V$, the natural maps $0 \rightarrow V, V \rightarrow 0$ induce a direct sum decomposition $F(V)=F(0) \oplus F^{\prime}(V)$, where $F^{\prime}$ is another endofunctor

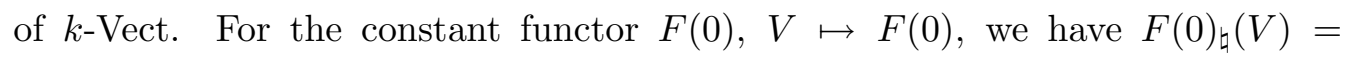
$F(0) \otimes T_{0}^{*}$, and Lemma 3.2 immediately implies that the additivization of this 
constant functor is trivial, so that $\operatorname{Add} .(F) \cong \operatorname{Add} .\left(F^{\prime}\right)$. Thus we may assume from the beginning that $F(0)=0$. Then applying $F_{\text {b }}$ pointwise to (3.11), we obtain a sequence

$$
0 \longrightarrow F_{\natural}^{C}\left(E_{1}\right) \longrightarrow F_{\natural}^{C}\left(E_{2}\right) \longrightarrow F_{\natural}^{C}\left(E_{3}\right) \longrightarrow 0
$$

in Fun $\left(\Gamma_{+} \times C, k\right)$ which is a complex - the composition map $F_{\natural}^{C}\left(E_{1}\right) \rightarrow F_{\natural}^{C}\left(E_{3}\right)$ is 0 . Thus it suffices to prove that if we denote by $H_{l}, l=1,2,3$, the homology of this complex, then $\operatorname{Tor}_{\Gamma_{+}}^{\cdot}\left(H_{l}, T\right)=0$. This fact can be proved pointwise, so that we may assume that $C=$ pt. But then we can split (3.11); we immediately see that $H_{1}=H_{3}=0$, and $\operatorname{Tor}_{\Gamma_{+}}^{\cdot}\left(H_{2}, T\right)=0$ by Lemma 3.4.

We will now apply additivization to compute stable homology. We will denote $\operatorname{Span}_{k}(V)=\overline{k[V]}$, the reduced $k$-linear span functor. We will use the Dold-Kan equivalence and the simplicial characterization of acyclic complexes described in Subsection 1.2.

Proposition 3.8. For any simplicial $k$-vector space $V . \in \operatorname{Fun}\left(\Delta^{o}, k\right)$, we have

$$
H_{\bullet}\left(\Delta^{o}, \operatorname{Add}_{\bullet}^{\Delta^{o}}\left(\operatorname{Span}_{k}\right)\left(V_{\bullet}\right)\right) \cong H_{\bullet+1}\left(\Delta^{o}, \operatorname{Add}_{\bullet}\left(\operatorname{Span}_{k}\right)^{\Delta^{o}}\left(V_{\bullet}[1]\right)\right),
$$

where $V_{.}[1] \in \operatorname{Fun}\left(\Delta^{o}, k\right)$ corresponds to the shift $V_{\bullet}[1] \in C^{\leq 0}(k)$ under the DoldKan equivalence. Moreover, if $V$. is a k-vector space $V$ considered as a constant simplicial vector space, then both sides are quasiisomorphic both to the homology of the complex Add. $\left(\operatorname{Span}_{k}\right)(V) \in \mathcal{D}^{-}(k)$ and to the stable homology St. $(V)$ of $V$.

Proof. For any $V . \in \operatorname{Fun}\left(\Delta^{o}, k\right)$, we have a natural short exact sequence

$$
0 \longrightarrow V_{\bullet} \longrightarrow \tilde{V}_{\bullet} \longrightarrow V_{\bullet}[1] \longrightarrow 0
$$

in $C^{\leq 0}(k) \cong \operatorname{Fun}\left(\Delta^{o}, k\right)$, where $\widetilde{V}$. is the cone of the identity map $V_{\text {. }} \rightarrow V_{\text {. }}$. This $\widetilde{V}$. is obviously homotopic to 0 , thus extends to a functor $\widetilde{V}_{\bullet}^{+} \in \operatorname{Fun}\left(\Delta_{+}^{o}, k\right)$. Therefore the additivization $\operatorname{Add}^{\Delta^{o}}\left(\operatorname{Span}_{k}\right)(\widetilde{V} \cdot)$ also extends to a object in $\mathcal{D}^{-}\left(\Delta_{+}^{o}, k\right)$, namely $\operatorname{Add}_{\bullet}^{\Delta_{+}^{o}}\left(\operatorname{Span}_{k}\right)\left(\widetilde{V}_{\bullet}^{+}\right)$, and we have

$$
H_{\bullet}\left(\Delta^{o}, \operatorname{Add}_{\bullet}^{\Delta^{o}}\left(\operatorname{Span}_{k}\right)(\widetilde{V} \cdot)=0,\right.
$$

which immediately implies (3.12) by Lemma 3.7. If $V \cdot=\tau^{*} V \in \operatorname{Fun}\left(\Delta^{o}, k\right)$ is a constant simplicial vector space, where $\tau: \Delta^{o} \rightarrow \mathrm{pt}$ is the projection to the point, then we taulogically have

$$
\operatorname{Add}^{\Delta^{o}}\left(\operatorname{Span}_{k}\right)\left(\tau^{*} V\right) \cong \tau^{*} \text { Add. }\left(\operatorname{Span}_{k}\right)(V),
$$


and for any complex $E . \in \mathcal{D}^{-}(k)$, we have $L^{\cdot} \tau_{!} \tau^{*} E$. $\cong E$. $\otimes L^{\bullet} \tau_{!} \tau^{*} k \cong E$. It remains to prove that for any $l \geq 0, H_{l}\left(\Delta^{o}, \operatorname{Add}^{\Delta^{o}}\left(\operatorname{Span}_{k}\right)\left(\tau^{*} V\right)\right)$ is isomorphic to the $l$-th stable homology group of $V$. By (3.12), we may equally well consider $H_{l+n}\left(\Delta^{o}, \operatorname{Add}_{\Delta^{o}}^{\Delta^{o}}\left(\operatorname{Span}_{k}\right)\left(\tau^{*} V[n]\right)\right)$ for some positive integer $n>0$. Take any $n>l$. Then by definition,

$$
\begin{aligned}
H_{l+n}\left(\Delta^{o}, \operatorname{Add}^{\Delta^{o}}\left(\operatorname{Span}_{k}\right)\left(\tau^{*}\right.\right. & V[n]))= \\
& =\operatorname{Tor}_{\Delta^{o} \times \Gamma_{+}}^{l+n}\left(\left(\operatorname{Span}_{k}\right)_{\natural}^{\Delta^{o}}\left(\tau^{*} V[n]\right), k \otimes T\right) \\
& =\operatorname{Tor}_{\Gamma_{+}}^{l+n}\left(L^{\bullet}(\mathrm{id} \times \tau) !\left(\operatorname{Span}_{k}\right)_{\bigsqcup^{o}}^{\Delta^{o}}\left(\tau^{*} V[n]\right), T\right) .
\end{aligned}
$$

For any $[m]_{+} \in \Gamma_{+}$, the complex $L^{\bullet}(\mathrm{id} \times \tau) !\left(\operatorname{Span}_{k}\right)_{\natural^{\circ}}^{\Delta^{o}}\left(\tau^{*} V[n]\right)\left([m]_{+}\right)$by definition computes the homology of the Eilenberg-MacLane space $K\left(V^{\oplus m}, n\right)$. Then the standard additivity property of the homology of the Eilenberg-MacLane spaces implies that $L^{\bullet}(\mathrm{id} \times \tau) !\left(\operatorname{Span}_{k}\right)_{\natural}^{\Delta^{o}}\left(\tau^{*} V[n]\right) \in \mathcal{D}^{-}\left(\Gamma_{+}, k\right)$ is linear in degrees between 0 and $2 n$. Thus the right-hand side of (3.13) is isomorphic to the $(l+n)$-th homology group $H_{l+n}(K(V, n), k)$ of the Eilenberg-MacLane space $K(V, n)$, which is in stable range.

Remark 3.9. The author has to confess that, being unaware of Pirashvili's work, he independently discovered Proposition 3.8 and other material in this Subsection in about 1997. The author never got around to writing this down, and later felt very stupid and very relieved, since in 1997 everything was already available in the literature - in the book form, no less.

3.3. The cube construction. Proposition 3.8 shows that for any $k$-vector space $V$, we have St. $(V) \cong \operatorname{Tor}_{\Gamma_{+}}^{\cdot}\left(\left(\operatorname{Span}_{k}\right)_{\natural}[V], T\right)$; thus to represent St. $(V)$ by a complex with good properties, we have to find a good projective resolution of $T \in \operatorname{Fun}\left(\Gamma_{+}^{o}, k\right)$. To do this, it is convenient to describe the category $\operatorname{Fun}\left(\Gamma_{+}^{o}, k\right)$ in a slightly different way using Lemma 3.2.

Denote by $\Gamma_{-}$the category of finite sets and surjective maps between them. By $[n] \in \Gamma_{-}, n \geq 1$, we will understand the set with $n$ elements.

Lemma 3.10. There exist equivalences of abelian categories $\operatorname{Fun}\left(\Gamma_{+}, k\right) \cong \operatorname{Fun}\left(\Gamma_{-}, k\right)$, $\operatorname{Fun}\left(\Gamma_{+}^{o}, k\right) \cong \operatorname{Fun}\left(\Gamma_{-}^{o}, k\right)$. 
Proof. We will only construct the first equivalence; the second one is obtained by a dual argument. Consider the functor Hom : $\Gamma_{-}^{o} \times \Gamma_{+} \rightarrow$ Sets defined by $\operatorname{Hom}\left([n] \times[m]_{+}\right)=\Gamma_{+}\left([n]_{+},[m]_{+}\right)$, and let $K^{\prime} \in \operatorname{Fun}\left(\Gamma_{-}^{o} \times \Gamma, k\right)$ be its $k$-linear span, $K^{\prime}\left([n] \times[m]_{+}\right)=k\left[\Gamma_{+}\left([n]_{+},[m]_{+}\right)\right]$. As in the proof of Lemma 3.2, say that a map $f:[n]_{+} \rightarrow[m]_{+}$is degenerate if the preimage of the distiguished point in $[m]_{+}$consist of more than the distinguished point in $[n]_{+}$. If $f:[n]_{+} \rightarrow[m]_{+}$ is degenerate, then so is its composition $f \circ g:[l]_{+} \rightarrow[m]_{+}$with any surjective $g:[l]_{+} \rightarrow[n]_{+}$; therefore degenerate maps form a subfunctor in Hom and span a $k$-linear subfunctor in $K^{\prime}$.

Let $K$ be the quotient by this subfunctor. One checks easily that for any $[n] \epsilon$ $\Gamma_{-}, K$ restricted to $[n] \times \Gamma_{+}$is isomorphic to the functor $T^{* \otimes n}$, and for any $[m]_{+} \in$ $\Gamma_{+}, K$ restricted to $\Gamma_{-} \times[m]_{+}$is isomorphic to the functor $s_{m} \in \operatorname{Fun}\left(\Gamma_{-}^{o}, k\right)$ represented by $[m] \in \Gamma_{-}$. Define a functor $\mathcal{K}: \operatorname{Fun}\left(\Gamma_{-}, k\right) \rightarrow \operatorname{Fun}\left(\Gamma_{+}, k\right)$ by the kernel $K$ - that is, set

$$
\mathcal{K}(L)=L \otimes_{\Gamma_{-}} K
$$

for any $L \in \operatorname{Fun}\left(\Gamma_{-}, k\right)$. Since $K$ restricts to a projective functor on every $\Gamma_{-} \times[m]$, the functor $\mathcal{K}$ is exact. We claim that it is an equivalence. To prove it, it suffices to prove that $\mathcal{K}$ sends a set of projective generators of $\operatorname{Fun}\left(\Gamma_{-}, k\right)$ to a set of projective generators of $\operatorname{Fun}\left(\Gamma_{+}, k\right)$, and is fully faithfull on this set. Indeed, a set of projective generators of $\operatorname{Fun}\left(\Gamma_{-}, k\right)$ is formed by functors $t_{n}^{*} \in \operatorname{Fun}\left(\Gamma_{-}, k\right)$ represented by all objects $[n] \in \Gamma_{-}$(we use $t_{n}^{*}$, not $t_{n}$ for consistency with earlier notation $\left.T, T^{*}\right)$. We tautologically have $\mathcal{K}\left(t_{n}^{*}\right)=K_{[n] \times \Gamma_{+}}=T^{* \otimes n}$, these do form a set of projective generators of $\operatorname{Fun}\left(\Gamma_{+}, k\right)$. Finally, $\mathcal{K}$ is fully faithfull on $\left\{t_{n}^{*}\right\}$ by Lemma 3.2 .

Remark 3.11. Lemma 3.10 seems to be well-known in folklore. I haven't been able to find an exact reference but I'm pretty sure that one exists, probably more than one. The statement is in fact completely parallel to the Dold-Kan equivalence, and the proofs are also very similar.

As a corollary of the proof, we see that the equivalence $\operatorname{Fun}\left(\Gamma_{+}^{o}, k\right) \cong \operatorname{Fun}\left(\Gamma_{-}^{o}, k\right)$ sends $T \in \operatorname{Fun}\left(\Gamma_{+}^{o}, k\right)$ to the functor $t_{1} \in \operatorname{Fun}\left(\Gamma_{-}^{o}, k\right)$ co-represented by $[1] \in \Gamma_{-}$. This functor is very small - we have $t_{1}([1])=k$, and $t_{1}([n])=0$ for $n \geq 2$. It is injective, but not projective. A set of projective generators of the category $\operatorname{Fun}\left(\Gamma_{-}^{o}, k\right)$ already appeared in the proof of Lemma 3.10; it is given by the 
functors $s_{n} \in \operatorname{Fun}\left(\Gamma_{-}^{o}, k\right)$ represented by objects $[n] \in \Gamma_{-}-$we have

$$
s_{n}([m])=k\left[\Gamma_{-}([m],[n])\right] .
$$

The functor $s_{1}$ is thus the constant functor $k \in \operatorname{Fun}\left(\Gamma_{-}, k\right)$. Moreover, $\operatorname{Hom}\left(s_{n}, s_{m}\right)=$ $k\left[\Gamma_{-}([n],[m])\right]$; in particular, every functor $s_{n}$ admits a canonical map to $s_{1}=k$. The functor $t_{1}$ is obviously a quotient of $s_{1}=k$, and moreover, we have an exact sequence

$$
s_{2} \longrightarrow s_{1} \longrightarrow t_{1} \longrightarrow 0 .
$$

Slightly more generally, assume given a small category $C$, a functor $P \in \operatorname{Fun}(C, k)$, and a map $\eta: P \rightarrow k$. Starting from these data, we can define a complex $\langle P ., d\rangle$ by taking $P_{i}=P^{\otimes i}$, and setting

$$
d_{i}=\sum_{1 \leq j \leq i}(-1)^{j} \mathrm{id}^{\otimes(j-1)} \otimes \eta \otimes \mathrm{id}^{\otimes(i-j)}
$$

for the differential $d_{i}: P_{i} \rightarrow P_{i-1}$.

Lemma 3.12. Let $P=s_{2}$, and let $\eta: P \rightarrow k$ be the canonical map $s_{2} \rightarrow s_{1}$. Then the complex $P$. is a projective resolution of $t_{1} \in \operatorname{Fun}\left(\Gamma_{-}^{o}, k\right)$.

Proof. We have to prove that $P$. is a resolution of $t_{1}$, and that every $P_{i}$ is projective. For the former, (3.14) gives a natural augmentation map $P$. $\rightarrow t_{1}$, and it suffices to prove that for any $[n] \in \Gamma_{-}$, the map $P .([n]) \rightarrow t_{1}([n])$ is a quasiisomorphism. If $n=1$, this is obvious, since $P_{1}([n])$ becomes $s_{2}([1])=0$ and therefore $P_{i}([n])=0$ for all $i \geq 1$. If $n \geq 2, t_{1}([n])=0$; thus we have to prove that $P_{\text {. }}([n])$ is acyclic. This is also obvious: we choose a section $\sigma: k \rightarrow P_{1}([n])$ of the surjective map $\eta: P_{1}([n]) \rightarrow k$, and notice that $\sigma \otimes \mathrm{id}^{\otimes i}$ is a contracting homotopy for the complex $P .([n])$.

To prove that $P_{i}$ is projective, it suffices to notice that $P_{i}=P^{\otimes i}=s_{2}^{\otimes i}$ evaluated at $[n] \in \Gamma_{-}$is by definition the linear span of the set of $i$-tuples of surjective maps from $[n]$ to [2]. We can combine such an $i$-tuple into a single map $[n] \rightarrow[2]^{i}=\left[2^{i}\right]$ and separate the $i$-tuples according to the image $S \subset\left[2^{i}\right]$ of this map; this gives a decomposition

$$
s_{2}^{\otimes i}=\bigoplus_{S \subset\left[2^{i}\right]} s_{|S|},
$$


where the indexing is over such subsets $S \subset\left[2^{i}\right]=[2]^{i}$ that for every $j, 1 \leq j \leq i$, the projection $p_{j}:[2]^{i} \rightarrow[2]$ onto the $j$-th coordinate induces a surjective map $p_{j}: S \rightarrow[2]$.

Thus for any finitely presented endofunctor $F$ of $k$-Vect, and for any $k$-vector space $V$, we can set

$$
Q .(F)(V)=F_{\natural}(V) \otimes_{\Gamma_{+}} \mathcal{K}\left(s_{2}^{\otimes \bullet}\right),
$$

where $\mathcal{K}: \operatorname{Fun}\left(\Gamma_{-}, k\right) \rightarrow \operatorname{Fun}\left(\Gamma_{+}, k\right)$ is the equivalence of Lemma 3.10. Then (3.15) induces a differential on the complex $Q .(F)(V)$, and the resulting complex represents the additivization Add. $(F)(V) \in \mathcal{D}^{-}(k)$. When $F(V)=\operatorname{Span}_{k}(V)=$ $\overline{k[V]}$, the reduced $k$-linear span functor, we denote $Q$. $\left(\operatorname{Span}_{k}\right)(V)$ simply by $Q$. $(V)$; then $Q .(V)$ is a complex which computes $\operatorname{St}(V)$. This is the EilenbergMacLane cube construction.

In principle, this is all that we will need to know about the cube construction; however, to simplify comparison with [LP], we will show in the remainder of this subsection how to compute $Q .(V)$ explicitly.

For any $[n]_{+} \in \Gamma_{+}$, denote by $S_{n} \in \operatorname{Fun}\left(\Gamma_{+}^{o}, k\right)$ the functor represented by $[n]_{+}$. This is a projective functor, and for any $F \in \operatorname{Fun}\left(\Gamma_{+}, k\right)$, we tautologically have

$$
F \otimes_{\Gamma_{+}} S_{n} \cong F\left([n]_{+}\right) .
$$

For any vector space $V$ and any integer $n \geq 0$, one denotes

$$
\begin{aligned}
& Q_{n}^{\prime}(V)=\left(\operatorname{Span}_{k}\right)_{\natural}(V) \otimes_{\Gamma_{+}} S_{2^{n}} \cong\left(\operatorname{Span}_{k}\right)_{\natural}(V)\left(\left[2^{n}\right]_{+}\right) \\
& \cong \overline{k\left[V \otimes T^{*}\left(\left[2^{n}\right]_{+}\right)\right]} \cong \overline{k\left[V^{\oplus 2^{n}}\right]} .
\end{aligned}
$$

In other words, we consider the vector space $V \otimes T^{*}\left(\left[2^{n}\right]_{+}\right)$, which a direct sum of $2^{n}$ copies of $V$ indexed by vertices of the $n$-cube $\left[2^{n}\right]=[2]^{n}$ - thus the "cube construction" - and $Q_{n}^{\prime}(V)$ is the reduced $k$-linear span of this vector space (considered as a pointed set). It is easy to check that $S_{2^{n}}$ decomposes as

$$
S_{2^{n}}=\bigoplus_{S \subset[2]^{n}} \mathcal{K}\left(s_{|S|}\right)
$$

where the sum is taken over all subsets $S \subset[2]^{n}$ of the cube. Thus the functor $\mathcal{K}\left(s_{2}^{\otimes n}\right)$ is a direct summand of $S_{2^{n}}$, and $Q_{n}(V)$ is a direct summand in $Q_{n}^{\prime}(V)$. The remainder corresponds to those summands in (3.19) which do not occur in (3.16) - namely, to those for which the subset $S \subset\left[2^{n}\right]$ is mapped onto a proper 
subset in [2] under at least one of the projections $p_{i}:\left[2^{n}\right] \rightarrow[2]$. "Proper subset" here of course must be a point, one point in [2] or the other one. Denote these points by $a, b \in[2]$, so that $[2]=\{a, b\}$; then for every $i$, we have two subsets $p_{i}^{-1}(a), p_{i}^{-1}(b) \subset[2]^{n}$, the faces of the cube, and the bad subsets in $[2]^{n}$ are those that lie entirely within one of those $2 n$ faces. Each face is itself a cube, of dimension $(n-1)$. One says that an element in $Q_{n}^{\prime}(V)$ is a slab if it lies in the image of the natural map $Q_{n-1}^{\prime}(V) \rightarrow Q_{n}^{\prime}(V)$ induced by the embedding $[2]^{n-1} \rightarrow[2]^{n}$ of one of the $2 n$ faces. One denotes by $N_{\text {. }}(V) \subset Q^{\prime} .(V)$ the subspace spanned by all the slabs; one then has $Q .(V)=Q^{\prime}(V) / N_{\bullet}(V)$.

The first differential in the complex $Q .(V)$ is obtained from (3.14), and one can check immediately that when written down explicitly in terms of $Q^{\prime}(V)$, it is exactly dual to (3.4) - we have

$$
d(a)=p_{1}(a)+p_{2}(a)-p_{12}(a)
$$

for any $a \in Q_{1}^{\prime}(V)=\overline{k(V \oplus V)}$, where $p_{1}, p_{2}: V \oplus V \rightarrow V$ are projections onto the first and the second summand, and $p_{12}: V \oplus V \rightarrow V$ is the summation map (here (3.14) gives $p_{12}(a)$, the two extra terms $p_{1}(a), p_{2}(a)$ appear because of the projection onto the direct summand $\left.Q_{1}(V) \subset Q_{1}^{\prime}(V)\right)$. Thus $Q$. $(V)$, in a sense, extends the short semi-exact sequence (3.4) to higher degrees. The differential in these higher degree terms is given by (3.15).

Remark 3.13. If one makes all of these constructions with $\Gamma_{+}$replaced by the category $\Delta$ - that is, if we impose linear order on our finite sets - then instead of the complex $Q .(V)$, we will get the normalized bar complex for the group $V$, and instead of the stable homology of the group $V$, we compute its usual homology. The terms of the bar complex are, of course, $k$-linear spans of the sets $V \times V \times \cdots \times V=V^{\oplus n}$ - in other words, of the sums of copies of $V$ indexed by vertices of a simplex instead of a cube (and we have to take out the degenerate simplices which correspond to slabs). For the bar construction, it makes no difference whether we normalize or not; for the cube construction, it is of crucial importance that we do normalize (if we don't, the functor $s_{2}$ in (3.14) gets replaced with $\mathcal{K}^{-1}\left(S_{2}\right)=s_{2} \oplus s_{1}^{\oplus 2}$, the $k$-vector space $P([1])$ is no longer trivial, and the complex $P$. stops being a resolution of $\left.t_{1} \in \operatorname{Fun}\left(\Gamma_{1}^{o}, k\right)\right)$. This parallel between the very standard bar construction and the very unusual cube construction is quite intriguing, and probably not completely understood. 
3.4. Multiplication. We now turn to multiplication. We start with the Segal's approach. Thus for any $k$-algebra $A$, we need complexes representing $\operatorname{St}(A)^{\otimes n} \in$ $\mathcal{D}^{-}(k)$, for all $n \geq 1$, and natural maps between them. The complexes are easily provided by the Künneth formula: we immediately deduce from Proposition 3.8 that for any $n \geq 2$, we have

$$
\operatorname{Tor}_{\Gamma_{+}^{n}}^{\cdot}\left(\left(\operatorname{Span}_{k}\right)_{\natural}(A)^{\bigotimes n}, T^{\bigotimes n}\right) \cong \operatorname{St}(A)^{\otimes n} .
$$

We now have to specify what natural maps, exactly, we want to construct.

The full set of symmetries and natural maps between various tensor powers $B^{\otimes n}$ of an associative unital algebra $B$ is conveniently encoded in a category that is defined as follows: objects are finite sets; a map is a map of finite sets and a linear order on the preimage of every point. This category variously appeared in the literature over the years and was known by different names ( $\Sigma$ in [GM, Exercize II.1.5], $\Delta S$ in [L, Chapter 6.1]); these days, it is sometimes called the category of non-commutative sets. To any associative unital $k$-algebra $B$, one associates a functor $\Sigma \rightarrow k$-Vect which sends a set with $n$ elements to $B^{\otimes n}$. The cyclic category $\Lambda$ is naturally a subcategory in $\Sigma$, and the $\sharp$-construction of Subsection 2.1 amount to restricting the universal functor associated to $B$ to $\Lambda \subset \Sigma$. Since in this paper, we are only interested in cyclic homology, we will not bother with the entire category $\Sigma$ and the universal functor, and we will directly construct a cyclic complex St. $(A)_{\sharp}$ which serves as its restriction to $\Lambda \subset \Sigma$.

To combine the category $\Lambda$ and the self-products $\Gamma_{+}^{n}$ needed for the Künneth formula (3.20), we use a version of the wreath product introduced in Subsection 2.2. We start with some generalities.

Assume given a unital monoidal object $C$ in Cat - that is, a strictly associative unital monoidal category. Applying the $\sharp$-construction, we obtain a functor $C_{\sharp}$ : $\Lambda \rightarrow$ Cat; using the Grothendieck construction, we produce a category cofibered over $\Lambda$ which we will call the co-wreath product of $C$ and $\Lambda$ and denote $\Lambda \int C$. The fiber of $\Lambda \int C$ over $[n] \in \Lambda$ is identified with the $n$-fold self-product $C^{n}$.

Remark 3.14. If $C$ is only associative in the usual sense - that is, it admits an associativity morphism which is not trivial - then $C_{\sharp}$ can be defined as a weak functor from $\Lambda$ to Cat, so that the Grothendieck construction still applies, and the co-wreath product $\Lambda \int C$ is well-defined. We will not need this. 
Consider the functor category $\operatorname{Fun}(C, k)$. The monoidal structure on $C$ induces a tensor structure on $\operatorname{Fun}(C, k)$ by the following rule.

Definition 3.15. For any two functors $F, G \in \operatorname{Fun}(C, k)$, their convolution product $F \circ G$ is defined as

$$
F \circ G=m_{!}(F \otimes G),
$$

where $m: C \times C \rightarrow C$ is the product functor.

One checks easily that the convolution product on $\operatorname{Fun}(C, k)$ is associative and unital (the unit is given by the functor $1_{C} \in \operatorname{Fun}(C, k)$ represented by the unit object $\left.1 \in C, 1_{C}(a)=k[C(1, a)]\right)$.

For any associative unital algebra $A$ in $\operatorname{Fun}(C, k)$ with respect to the convolution product, we obtain by adjunction a canonical map

$$
A \otimes A \rightarrow m^{*} A
$$

and its iterates $A^{\bigotimes n} \rightarrow m_{n}^{*} A$, where $m_{n}: C^{n} \rightarrow C$ is the $n$-fold product functor for the monoidal structure on $C$. Combining all these maps together, we get the following generalization of the $\sharp$-construction: for any associative unital algebra $A \in\langle\operatorname{Fun}(C, k), \circ\rangle$, we can construct a functor $A_{\sharp} \in \operatorname{Fun}\left(\Lambda \int C, k\right)$ whose restriction to the fiber $C^{n} \subset \Lambda \int C$ over $[n] \in \Lambda$ is the $n$-fold self-product $A^{\bigotimes n}$.

For any monoidal unital category $C$, the opposite category $C^{o}$ is of course also monoidal and unital, so that all of the constructions above apply. Assume given an associative unital algebra $A \in\langle\operatorname{Fun}(C, k), \circ\rangle$ and an associative unital algebra $B \in\left\langle\operatorname{Fun}\left(C^{o}, k\right), \circ\right\rangle$. Then the convolution $A \otimes_{C} B$ is naturally an algebra: the product is given by the natural map

$$
\left(A \otimes_{C} B\right)^{\otimes 2} \cong A^{\bigotimes 2} \otimes_{C^{2}} B^{\bigotimes 2} \rightarrow m_{!}\left(A^{\otimes 2}\right) \otimes_{C} m_{!}^{o}\left(B^{\bigotimes 2}\right) \rightarrow A \otimes_{C} B,
$$

where we have used the Künneth formula on the left-hand side, and the map in the middle is the natural map (1.5). This algebra $\left(A \otimes_{C} B\right)$ is associative and unital, so that we can form the object $\left(A \otimes_{C} B\right)_{\sharp} \in \operatorname{Fun}(\Lambda, k)$. On the other hand, we can obtain the same object by doing the convolution relatively over $\Lambda$ : using the natural map (1.5), we can generalize the coend functor (1.2) to obtain a bilinear functor from $\operatorname{Fun}\left(\Lambda \int C, k\right) \times \operatorname{Fun}\left(\Lambda \int C^{o}, k\right)$ to $\operatorname{Fun}(\Lambda, k)$ which we denote by $\otimes_{\Lambda} \int C / \Lambda$, and we have a natural isomorphism

$$
\left(A \otimes_{C} B\right)_{\sharp} \cong A_{\sharp} \otimes_{\Lambda} \int C / \Lambda B_{\sharp} .
$$


Let now $C=\Gamma_{+}$, the category of finite pointed sets. The smash product introduced in (3.5) defines a functor $\Gamma_{+} \times \Gamma_{+} \rightarrow \Gamma_{+}$; this functor is in fact strictly associative, so that $\Gamma_{+}$is a monoidal object in Cat. The object $[1]_{+} \in \Gamma_{+}$is obviously a unit object. Thus we can form the co-wreath product $\Lambda \int \Gamma_{+}$. Dualizing, we obtain a monoidal structure on $\Gamma_{+}^{o}$ and the co-wreath product $\Lambda \int \Gamma_{+}^{o}$.

Lemma 3.16. The object $T \in \operatorname{Fun}\left(\Gamma_{+}^{o}, k\right)$ is an associative unital algebra with respect to the convolution product.

Proof. By adjunction, to construct the multiplication map $m_{!}(T \otimes T) \rightarrow T$, it suffices to construct a map $T \otimes T \rightarrow m^{*} T$. Recall that for any $[n]_{+} \in \Gamma, T\left([n]_{+}\right)$is by definition the reduced $k$-linear span of the pointed set $[n]_{+}$. Therefore $T\left([n]_{+} \wedge\right.$ $\left.[m]_{+}\right)$is canonically isomorphic to $T\left([n]_{+}\right) \otimes T\left([m]_{+}\right)$, so that $m^{*} T \cong T \otimes T$. We leave it to the reader to check that this product is unital and associative.

Definition 3.17. An endofunctor $F$ of the category $k$-Vect is called multiplicative if for any two vector spaces $V_{1}, V_{2}$ we are given a map $F\left(V_{1}\right) \otimes F\left(V_{2}\right) \rightarrow F\left(V_{1} \otimes V_{2}\right)$, these maps are functorial in $V_{1}$ and $V_{2}$, and satisfy the natural associativity condition: both possible ways to combine them to a map $F\left(V_{1}\right) \otimes F\left(V_{2}\right) \otimes F\left(V_{3}\right) \rightarrow$ $F\left(V_{1} \otimes V_{2} \otimes V_{3}\right)$ give the same result.

Assume given such a multiplicative functor $F$, and consider the functor $F_{\natural}(V) \in$ $\operatorname{Fun}\left(\Gamma_{+}, k\right), F_{\natural}(V)\left([n]_{+}\right)=F\left(T^{*}\left([n]_{+}\right) \otimes V\right)$. Then for any two $k$-vector spaces $V_{1}, V_{2}$, the multiplication maps

$$
\begin{aligned}
& F\left(T^{*}\left([n]_{+}\right) \otimes V_{1}\right) \otimes F\left(T^{*}\left([m]_{+}\right) \otimes V_{2}\right) \rightarrow \\
& \quad \rightarrow F\left(T^{*}\left([n]_{+}\right) \otimes T^{*}\left([m]_{+}\right) \otimes V_{1} \otimes V_{2}\right) \cong F\left(T^{*}\left([n]_{+} \wedge[m]_{+}\right) \otimes V_{1} \otimes V_{2}\right)
\end{aligned}
$$

combine together to give a map

$$
F_{\natural}\left(V_{1}\right) \otimes F_{\natural}\left(V_{2}\right) \rightarrow m^{*} F_{\natural}\left(V_{1} \otimes V_{2}\right),
$$

and this satisfies the natural associativity condition. Therefore if $A$ is an associative and unital $k$-algebra, the functor $F_{\natural}(A) \in \operatorname{Fun}\left(\Gamma_{+}, k\right)$ is naturally an associative unital algebra in $\operatorname{Fun}\left(\Gamma_{+}, k\right)$ with respect to the convolution. Thus we can form an object $F_{\sharp}(A)_{\sharp} \in \operatorname{Fun}\left(\Lambda \int \Gamma_{+}, k\right)$.

Definition 3.18. For any associative unital $k$-algebra $A$, we let

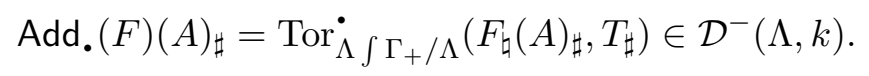


For the reduced $k$-linear span functor $\mathrm{Span}_{k}$, we denote

$$
\operatorname{Add} .\left(\operatorname{Span}_{k}\right)(A)_{\sharp}=\operatorname{St} .(A)_{\sharp} \in \mathcal{D}^{-}(\Lambda, k) \text {. }
$$

This is the cyclic complex that plays the role of $\operatorname{St}(A)_{\sharp}$ in the Segal's approach.

Using the cube construction, we can do better - we can actually put some DG algebra structure on the complex $Q$. $(F)(A)$, so that the cyclic complex $Q .(F)(A)_{\sharp}$ represents Add. $(F)(A)_{\sharp} \in \mathcal{D}^{-}(\Lambda, k)$. Indeed, as we see from (3.21), all we need to do for this is to show that the projective resolution $P$. of $t_{1} \in \operatorname{Fun}\left(\Gamma_{-}^{o}, k\right)$ constructed in Subsection 3.3 goes, under the equivalence $\mathcal{K}: \operatorname{Fun}\left(\Gamma_{-}^{o}, k\right) \rightarrow$ $\operatorname{Fun}\left(\Gamma_{+}^{o}, k\right)$, to a DG algebra resolution of $T=\mathcal{K}\left(t_{1}\right)$, considered as an algebra with respect to the convolution tensor product on $\operatorname{Fun}\left(\Gamma_{+}^{o}, k\right)$. But by construction, $P$. is tautologically a DG algebra with respect to the usual tensor product on $\operatorname{Fun}\left(\Gamma_{-}^{o}, k\right)$, so that it suffices to use the following fact.

Lemma 3.19. The equivalence $\mathcal{K}: \operatorname{Fun}\left(\Gamma_{-}^{o}, k\right) \rightarrow \operatorname{Fun}\left(\Gamma_{+}^{o}, k\right)$ constructed in Lemma 3.10 sends the usual tensor product to the convolution tensor product that is, for any two functors $F_{1}, F_{2} \in \operatorname{Fun}\left(\Gamma_{-}^{o}, k\right)$ we have natural isomorphism

$$
\mathcal{K}\left(F_{1} \otimes F_{2}\right) \cong \mathcal{K}\left(F_{1}\right) \circ \mathcal{K}\left(F_{2}\right)
$$

where the convolution $\circ$ is taken with respect to the monoidal structure on $\Gamma_{+}$ given by the smash product $\wedge$.

Proof. Recall that the equivalence $\mathcal{K}$ is represented by a kernel $K: \Gamma_{-} \times \Gamma_{+}^{o} \rightarrow$ $k$-Vect. It is more convenient to use the inverse equivalence which is represented by the dual kernel $K^{*} \in \operatorname{Fun}\left(\Gamma_{-}^{o} \times \Gamma_{+}, k\right)$. The usual tensor product on $\operatorname{Fun}\left(\Gamma_{-}^{o}, k\right)$ is of course obtained by restriction to the diagonal $\delta: \Gamma_{-}^{o} \rightarrow \Gamma_{-}^{o} \times \Gamma_{-}^{o}, F_{1} \otimes F_{2}=$ $\delta^{*}\left(F_{1} \otimes F_{2}\right)$. Thus the functor $F_{1}, F_{2} \mapsto \mathcal{K}^{-1}\left(F_{1}\right) \otimes \mathcal{K}^{-1}\left(F_{2}\right)$ is represented by the kernel $\delta^{*}\left(K^{*} \otimes K^{*}\right) \in \operatorname{Fun}\left(\Gamma_{-}^{o} \times \Gamma_{+} \times \Gamma_{+}, k\right)$ - that is, we have

$$
\mathcal{K}^{-1}\left(F_{1}\right) \otimes \mathcal{K}^{-1}\left(F_{2}\right)=\delta^{*}\left(K^{*} \otimes K^{*}\right) \otimes_{\Gamma_{+} \times \Gamma_{+}} F_{1} \otimes F_{2} .
$$

On the other hand, by (1.4) applied to $m: \Gamma_{+} \times \Gamma_{+} \rightarrow \Gamma_{+}$, we have

$$
\mathcal{K}^{-1}\left(F_{1} \circ F_{2}\right)=K^{*} \otimes_{\Gamma_{+}} m_{!}\left(F_{1} \otimes F_{2}\right)=m^{*} K^{*} \otimes_{\Gamma_{+} \times \Gamma_{+}}\left(F_{1} \otimes F_{2}\right),
$$

where $m: \Gamma_{+} \times \Gamma_{+} \rightarrow \Gamma_{+}$is the smash product. Thus to prove the lemma, we have to construct an isomorphism $\delta^{*}\left(K^{*} \otimes K^{*}\right) \cong m^{*} K$. Explicitly, for any 
$[n] \in \Gamma_{-},\left[m_{1}\right]_{+},\left[m_{2}\right]_{+} \in \Gamma_{+}$, we have to construct an identification

$$
k\left[\operatorname{Maps}\left([n],\left[m_{1}\right]\right) \times \operatorname{Maps}\left([n],\left[m_{1}\right]\right)\right]^{*} \cong k\left[\operatorname{Maps}\left([n],\left[m_{1}\right] \times\left[m_{2}\right]\right)\right]^{*},
$$

where Maps $([a],[b])$ means the set of all maps from the finite set $[a]$ to the finite set $[b]$ (equivalently, all non-degenerate maps from $[a]_{+}$to $[b]_{+}$). This is rather tautological.

Remark 3.20. One can easily generalize Lemma 3.19 to obtain an equivalence $\operatorname{Fun}\left(\Lambda \int \Gamma_{+}^{o}, k\right) \cong \operatorname{Fun}\left(\Gamma_{-} \int \Lambda, k\right)$, where $\Gamma_{-} \int \Lambda$ is the wreath product as in Subsection 2.2 (which does not require a monoidal structure on $\Gamma_{-}$). This gives another way to obtain the cyclic complex $Q .(A)_{\sharp}$. However, we must caution the reader that if one replaces the categories $\Gamma_{+}^{o}, \Gamma_{-}^{o}$ in Lemma 3.19 with the opposite categories $\Gamma_{+}, \Gamma_{-}$, the statement becomes completely false (the symmetry is broken because of $m_{\text {! }}$ in Definition 3.15, which should not be replaced with $m_{*}$ ). For this reason, when dealing with the left-hand side of (3.22) - that is, with the algebra structure on $F_{\natural}(A)$ - one has to stick to smash products and pointed sets.

\section{Cartier map - the general Case.}

We now assume given an associative algebra $A$ over a perfect field $k$ of characteristic char $k=p>0$, and we construct the Cartier map for $A$. We will use the cube construction, since it slightly simplifies things. We will also assume throughout this section that the category $A$-bimod of $A$-bimodules has finite homological dimension.

4.1. Generalized Cartier map. Recall that for any endofunctor $F$ of the category $k$-Vect, we have its additivization Add. $(F): k$-Vect $\rightarrow \mathcal{D}^{-}(k)$ which can be presented by an explicit complex $Q .(F)$; if $F$ is multiplicative in the sense of Definition 3.17, then $Q .(F)(A)$ is a DG algebra over $k$.

For the reduced $k$-linear span functor $\operatorname{Span}_{k}$, we denote $Q .\left(\operatorname{Span}_{k}\right)(A)=Q .(A)$; this is a DG algebra realization of the associative algebra $\operatorname{St}(A) \in \mathcal{D}^{-}(k)$. Explicitly, by Proposition 3.8, the homology of the complex $Q .(A)$ is isomorphic to the stable homology of $A$ considered as a vector space. In low degrees, we have

$$
H_{i}(Q .(A))= \begin{cases}A \otimes_{\mathbb{Z}} k, & i=0,1, \\ 0, & 1<i<2 p-2 .\end{cases}
$$


In particular, the homology of $Q .(A)$ - in fact, the complex $Q .(A)$ itself - do not depend on the $k$-vector structure on $A$, only on the abelian group structure. This is not very convenient for our purposes. To correct the situation, assume from now that the perfect field $k$ is actually finite. For any $k$-vector space $V$, the multiplicative group $k^{*}$ acts on $\operatorname{Span}_{k}(V)=\overline{k[V]}$ by

$$
\lambda \cdot \sum a_{i}\left[v_{i}\right]=\sum \lambda^{-1} a_{i}\left[\lambda v_{i}\right], \quad \lambda \in k^{*}, a_{i} \in k, v_{i} \in V .
$$

Consider the quotient $\overline{\operatorname{Span}_{k}}(V)=\left(\operatorname{Span}_{k}(V)\right)_{k^{*}}$ obtained by taking the coinvariants with respect to the $k^{*}$-action, and let $\bar{Q} .(V)=Q$. $\left(\overline{\operatorname{Span}_{k}}\right)(V)$. Since the order of the group $k^{*}$ is coprime to $p=$ char $k$, we have $\bar{Q} .(V)=(Q .(V))_{k^{*}}$; in particular, (4.1) implies that the 0-th and 1-st homology of the complex $\bar{Q}$. $(V)$ is isomorphic to $V$. For an associative $k$-algebra $A, \bar{Q} .(A)$ is a DG quotient algebra of $Q .(A)$, and we have a natural augmentation map $\bar{Q} .(A) \rightarrow A$ which is a quasiisomorphism in degree 0 .

Another non-additive functor that we will need is the $p$-power functor. We denote it by Pow, Pow $(V)=V^{\otimes p}$, and we denote $Q$. $(\operatorname{Pow})(V)=P .(V)$. The functor Pow is obviously multiplicative. Moreover, the $p$-power $V^{\otimes p}$ carries a natural action of the cyclic group $\mathbb{Z} / p \mathbb{Z}$, so that Pow can be considered as a functor from $k$-Vect to $k$-linear representations of $\mathbb{Z} / p \mathbb{Z}$. One checks easily that the cube construction is sufficiently functorial, so that the DG algebra $P$. $(A)$ inherits a $\mathbb{Z} / p \mathbb{Z}$-action (more generally, for any small category $C$ and any functor $F: k$-Vect $\rightarrow \operatorname{Fun}(C, k)$, the cube construction generalizes immediately to give a functor $Q .(F)$ from $k$-Vect to complexes in $\operatorname{Fun}(C, k))$.

Lemma 4.1. For any $k$-vector space $V$, we have a $\mathbb{Z} / p \mathbb{Z}$-equivariant isomorphism $P_{.}(V) \cong V^{\otimes p} \otimes P_{.}(k)$, and if $V=A$ is an associative $k$-algebra, then this isomorphism is compatible with the algebra structures. The alegbra $P .(k)$ is a free assoative algebra generated by $P_{1}(k)$, which is a free module over $k[\mathbb{Z} / p \mathbb{Z}]$.

Proof. The isomorphism $P .(V) \cong V^{\otimes p} \otimes P$. $(k)$ is immediately obvious from the explicit form of the cube construction given in Subsection 3.3, (3.18) and the next paragraph. We also see that $P_{0}(k)=k$, and that $P_{1}(k)=k\left[S^{p} \backslash S\right]$, where $S=\{0,1\}$ is the set with two elements, and $S \subset S^{p}$ is the diagonal embedding. The action of $\mathbb{Z} / p \mathbb{Z}$ on $S^{p} \backslash S$ is free, so that $P_{1}(k)$ is a free $k[\mathbb{Z} / p \mathbb{Z}]$-module. The algebra $P .(k)$ is isomorphic to the free $k$-algebra generated by $k\left[S^{p}\right]$, modulo the 
relations given by $k[S] \subset k\left[S^{p}\right]$; counting the dimensions, we check that $P_{\text {. }}(k)$ is indeed freely generated by $P_{1}(k)$.

We see that for any $i \geq 1, P_{i}(V) \cong P_{i}(k) \otimes V^{\otimes p}$ is a free $k[\mathbb{Z} / p \mathbb{Z}]$-module, so that the Tate homology $\check{H}_{\text {. }}\left(\mathbb{Z} / p \mathbb{Z}, P_{i}(V)\right)$ vanishes. We would like to deduce that the natural map $V^{\otimes p} \cong P_{0}(V) \rightarrow P_{\text {. }}(V)$ induces an isomorphism of Tate homology. Unfortunately, this is not true (in fact, the complex $P .(V)$ is acyclic). The problem is that the complex $P_{.}(V)$ is infinite, and the relevant spectral sequence does not converge. To correct this problem, we have to consider $P$. $(V)$ as a filtered complex, with the stupid filtration, and to redefine Tate homology and periodic cyclic homology.

Definition 4.2. For any filtered object $V . \in \mathcal{D F}(k[\mathbb{Z} / p \mathbb{Z}] \bmod )$ in the derived category of $k[\mathbb{Z} / p \mathbb{Z}]$-modules, the filtered Tate homology $\check{H}_{\bullet}(\mathbb{Z} / p \mathbb{Z}, E$. $)$ is given by

$$
\check{H}_{\bullet}^{F}\left(\mathbb{Z} / p \mathbb{Z}, E_{\bullet}\right)=\lim _{\rightarrow} \check{H}_{\bullet}\left(\mathbb{Z} / p \mathbb{Z}, F_{i} E_{\bullet}\right) .
$$

For any integer $n \geq 1$ and any object $\left\langle E_{\bullet}, F.\right\rangle \in \mathcal{D F}\left(\Lambda_{n}, k\right)$, the filtered periodic cyclic homology $H P .(E$. $)$ is given by

$$
H P^{F}\left(E_{\bullet}\right)=\lim _{\rightarrow} H P_{\bullet}\left(F_{i} E_{\bullet}\right) .
$$

Now, for our algebra $A, P .(A)$ is a DG algebra equipped with an action of the cyclic group $\mathbb{Z} / p \mathbb{Z}$. As in Section 2 , the $\sharp$-construction gives a complex $P$. $(A)_{\sharp}$ in the category $\operatorname{Fun}\left(\mathrm{B}_{p}, k\right)$, which we equip with the stupid filtration. We can restrict it to $\Lambda_{p}$ by means of the embedding $\lambda: \Lambda_{p} \rightarrow \mathrm{B}_{p}$, as in Lemma 2.7, and obtain an object $\lambda^{*} P$. $(A)_{\sharp} \in \mathcal{D F}\left(\Lambda_{p}, k\right)$.

Lemma 4.3. For any associative algebra $A$ such that the category A-bimod has finite homological dimension, every term of the complex $\lambda^{*}\left(P .(A)_{\sharp}\right)$ is small in the sense of Definition 1.18, and the embedding $A^{\otimes p}=P_{0}(A) \rightarrow P_{\bullet}(A)$ induces an isomorphism

$$
H P .\left(\lambda^{*}\left(A^{\otimes p}\right)_{\sharp}\right) \rightarrow H P^{F}\left(\lambda^{*} P .(A)_{\sharp}\right) .
$$

Proof. For the first claim, the differential in the complex $P .(A)$ is irrelevant, so that we may consider $P .(A)$ as a graded algebra. As in Lemma 2.9, it suffices to prove that the bar resolution $C_{\bullet}\left(P_{\bullet}(A)\right)$ of the diagonal bimodule $P_{\bullet}(A)$ is 
effectively finite as a graded simplicial $\mathbb{Z} / p \mathbb{Z}$-equivariant $P$. $(A)$-bimodule. But $P_{.}(A) \cong A^{\otimes p} \otimes P_{.}(k)$, so that

$$
C_{.}\left(P_{.}(A)\right) \cong C_{.}\left(A^{\otimes p}\right) \otimes C .(k) .
$$

The first factor is effectively finite by Corollary 1.5 (ii). The second factor is effectively finite since $P$. $(k)$ is a free algebra generated by a free $\mathbb{Z} / p \mathbb{Z}$-modules as in the proof of Lemma 2.9, for any $m \geq 0$ the truncation $L^{\bullet} \tau_{!} F^{m} C .(P .(k))$ is concentrated in degree $m$, and moreover, is free as a module over $k[\mathbb{Z} / p \mathbb{Z}]$, thus has finite homological dimension as a $\mathbb{Z} / p \mathbb{Z}$-equivariant $P$. $(k)$-bimodule. Then the whole product is effectively finite by Corollary 1.5 (i), which finishes the proof of the first claim. The second claim immediately follows by induction on $i$ in Definition 4.2. Indeed, for any set of $\mathbb{Z} / p \mathbb{Z}$-modules $V_{1}, \ldots, V_{n}$ at least one of which is free, the product $V_{1} \otimes \cdots \otimes V_{n}$ is itself free; thus by Lemma 4.1, for any $i \geq 1$ the $i$-th term of the complex $\lambda^{*} P$. $(A)_{\sharp}$ satisfies the equivalent conditions of Lemma 1.15, and therefore has no periodic cyclic homology by Lemma 1.19.

We can now define our generalized Cartier map. For any vector space $V$, we can extend the map $V \rightarrow V^{\otimes p}, v \mapsto v^{\otimes p}$ to a $k$-linear map $\Phi: \overline{k[V]} \rightarrow V^{\otimes p}$, so that we have a map $\Phi: \operatorname{Span}_{k} \rightarrow$ Pow. This map is obviously $\mathbb{Z} / p \mathbb{Z}$-invariant; if we replace $V$ in the left-hand side with its Frobenius twist $V^{(1)}$, then it factors through a functorial $k$-linear map $\bar{\Phi}: \overline{\operatorname{Span}_{k}}\left(V^{(1)}\right) \rightarrow \operatorname{Pow}(V)$ and becomes compatible with the multiplicative structures and the stupid filtrations.

Definition 4.4. The generalized Frobenius map for the associative algebra $A$ is the $\mathbb{Z} / p \mathbb{Z}$-invariant map of filtered DG algebras

$$
\varphi=\bar{Q} .(\bar{\Phi}): \bar{Q} .\left(A^{(1)}\right) \rightarrow P .(A),
$$

where $\bar{\Phi}:(\overline{k[V]})_{k^{*}} \rightarrow V^{\otimes p}$ is the natural map induced by $[v] \mapsto v^{\otimes p}$.

Now, by Lemma 1.14 and Lemma 4.3, we canonically have

$$
H P^{F}\left(\lambda^{*} P .(A)_{\sharp}\right) \cong H P .(A),
$$

as in Proposition 2.10 (recall that we assume throughout that $A$-bimod has finite homological dimension). On the other hand, by Lemma 1.16, we have

$$
H P_{.}^{F}\left(\pi^{*} \bar{Q} .\left(A^{(1)}\right)_{\sharp}\right) \cong H H_{\bullet}\left(\bar{Q} .\left(A^{(1)}\right)_{\sharp}\right)((u)) \cong H H_{\bullet}\left(\bar{Q} .\left(A^{(1)}\right)\right)((u)) .
$$


Since Hochschild homology commutes with direct limits, there is no need to redefine it as in Definition 4.2 and $\bar{Q} .\left(A^{(1)}\right)$ in the right-hand side can be safely treated simply as a DG algebra, with no regards to filtrations.

Definition 4.5. The generalized Cartier map for the algebra $A$ is the map

$$
C=H P .\left(\lambda^{*} \varphi_{\sharp}\right): H H_{\bullet}\left(\bar{Q} .\left(A^{(1)}\right)\right)((u)) \rightarrow H P .(A)
$$

induced by the generalized Frobenius map $\varphi$.

The generalized Cartier map is defined in a completely canonical way and for any algebra $A$ of finite homological dimension, but it has no chance to be an isomorphism. For our applications, we need a stripped-down version of it which is an isomorphism. This of course would require some choices and some conditions on the algebra $A$. Our strategy is to reduce everything to the study of the DG algebra $Q .(A)$. This is sufficient for the following reason. By a $D G$ splitting

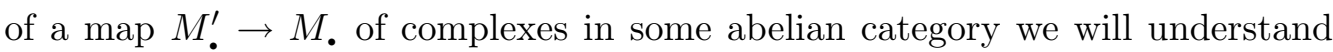
a complex $\widetilde{M}_{\text {. }}$ and a map $\widetilde{B}$. $\rightarrow B_{\text {. }}^{\prime}$ such that the composition $\widetilde{M}$. $\rightarrow M_{\text {. is }}$ a surjective quasiisomorphism. By a of a DG algebra map $B_{\text {. }}^{\prime} \rightarrow B$. we will understand a DG splitting $\left\langle\widetilde{B}_{\bullet}, s\right\rangle$ of the corresponding map of complexes such that $\widetilde{B}$. is a DG algebra, and $s$ is a DG algebra map.

Lemma 4.6. Assume given a $k$-vector space $V$ and a $D G$ splitting $s: V . \rightarrow \bar{Q}$. $(V)$ of the augmentation projection $\bar{Q} .(V) \rightarrow V$. Then the composition map

$$
V_{\bullet}^{(1)} \stackrel{s}{\longrightarrow} \bar{Q}_{\bullet}\left(V^{(1)}\right) \stackrel{\bar{\Phi}}{\longrightarrow} P_{\bullet}(V)
$$

induces a quasiisomorphism of filtered Tate homology groups $\check{H}_{\text {. }}^{F}(\mathbb{Z} / p \mathbb{Z},-)$.

Proof. By Lemma 4.1, we have $\check{H}$. $\left(\mathbb{Z} / p \mathbb{Z},(P .(V))_{i}\right)=0$ for any $i \geq 1$, so that, by virtue of Definition 4.2 , we have

$$
\check{H}_{\bullet}^{F}(\mathbb{Z} / p \mathbb{Z}, P .(V)) \cong \check{H}_{\bullet}\left(\mathbb{Z} / p \mathbb{Z}, V^{\otimes p}\right) .
$$

By Lemma 2.3, this is a free module over the Tate cohomology algebra $\check{H}^{\bullet}(\mathbb{Z} / p \mathbb{Z}, k)$ $=k\left[u, u^{-1}, \varepsilon\right]$ (here $\varepsilon \in H^{1}(\mathbb{Z} / p \mathbb{Z}, k)$ is a generator of degree 1$)$. The same is obviously true for $\check{H}^{F}\left(\mathbb{Z} / p \mathbb{Z}, V^{(1)}\right) \cong V^{(1)} \otimes \check{H}_{.}(\mathbb{Z} / p \mathbb{Z}, k)$. Therefore it suffices to consider the 0 -th homology groups $\check{H}_{0}(\mathbb{Z} / p \mathbb{Z},-)$. But $\check{H}_{0}^{F}(\mathbb{Z} / p \mathbb{Z}, P .(V))$ is concentrated in degree zero, so that the map

$$
\check{H}_{0}^{F}\left(\mathbb{Z} / p \mathbb{Z}, \bar{Q} .\left(V^{(1)}\right)\right) \rightarrow \check{H}_{0}^{F}(\mathbb{Z} / p \mathbb{Z}, P .(V))
$$


factors through the $n$-th tensor power of the augmentation map $\bar{Q}$. $\left(V^{(1)}\right) \rightarrow V^{(1)}$, and we are done by Lemma 2.3 .

Proposition 4.7. For any $D G$ splitting $s: A . \rightarrow \bar{Q}_{\bullet}(A)$ of the augmentation $\bar{Q} .(A) \rightarrow A$, the composition map

$$
H H_{\bullet}\left(A_{\bullet}^{(1)}\right)((u)) \stackrel{s}{\longrightarrow} H H_{\bullet}\left(\bar{Q} .\left(A^{(1)}\right)\right)((u)) \stackrel{C}{\longrightarrow} H P .(A)
$$

from $H_{\bullet}\left(A^{(1)}\right)((u)) \cong H_{\bullet}\left(A_{\bullet}^{(1)}\right)((u))$ to $H P .(A)$ is an isomorphism in all degrees.

Proof. By our assumption, the category $A$-bimod has finite homological dimension. Then as in the proof of Proposition 2.10, it suffices to prove that for any $n \geq 1$, the map

$$
A_{\bullet}^{(1) \otimes n} \stackrel{s^{(1) \otimes n}}{\longrightarrow} Q .\left(A^{(1)}\right)^{\otimes n} \stackrel{\varphi^{\otimes n}}{\longrightarrow} P .(A)^{\otimes n}
$$

becomes an isomorphism after passing to the filtered Tate homology of the group $\mathbb{Z} / p \mathbb{Z}$. We have $P_{.}(A)^{\otimes n} \cong A^{\otimes p n} \otimes P_{.}(k)^{n}, P_{.}\left(A^{\otimes n}\right) \cong A^{\otimes p n} \otimes P_{.}(k)$, and the multiplication map $m: P .(k)^{\otimes n} \rightarrow P$. $(k)$ is an isomorphism in degree 0. By Lemma 4.1, the filtered Tate homology of both $P .(A)^{\otimes n}$ and $P .\left(A^{\otimes n}\right)$ only depends on the degree-0 term $A^{\otimes p n}$, so that the map id $\otimes m: P .(A)^{\otimes n} \rightarrow P_{.}\left(A^{\otimes n}\right)$ induces an isomorphism on filtered Tate homology. Thus it suffices to prove that the map

$$
s^{(1) \otimes n} \circ \varphi^{\otimes n} \circ(\text { id } \otimes m): A_{.}^{(1) \otimes n} \rightarrow P .\left(A^{\otimes n}\right)
$$

induces an isomorphism on filtered Tate homology. This is Lemma 4.6, with $V=A^{\otimes n}$ and $V_{.}=A_{\bullet}^{\otimes n}$.

4.2. Splitting at first order. By virtue of Proposition 4.7, in order to construct a Cartier-type isomorphism for an algebra $A$, we have to find a DG splitting of the augmentation $\bar{Q}$. $(A) \rightarrow A$. We now turn to the study of the DG algebra $\bar{Q} .(A)$.

For any vector space $V$, consider the canonical filtration of the complex $\bar{Q} .(V)$; then the associated graded quotient gr. $\bar{Q} .(V)$ is quasiisomorphic to gr. $\bar{Q} .(k) \otimes V$, where, as we know from Proposition 3.8 , gr. $\bar{Q} .(k) \cong \mathrm{St}_{.}^{k^{*}}(k)$ is dual to the Steenrod algebra - the algebra of $k^{*}$-equivariant (or equivalently, $k$-linear) cohomological operations with coefficients in $k$. Explicitly, the quasiisomorphism 
$\mathrm{St}_{.}^{k^{*}}(k) \otimes V \rightarrow$ gr. $\bar{Q} .(V)$ is induced by the natural multiplication map $\bar{Q} .(k) \otimes$ $\bar{Q} .(V) \rightarrow \bar{Q} .(V \otimes k)=\bar{Q}$. $(V)$. If $V=A$ is an associative algebra, so that $\bar{Q}$. $(A)$ is a DG algebra, then the canonical filtration is compatible with the DG algebra structure, and gr. $\bar{Q} .(A) \cong \mathrm{St}^{k^{*}}(k) \otimes A$ is a DG algebra quasiisomorphism.

For any $l \geq 0$, we will denote by $\bar{Q}_{\leq l}(V)$ the $l$-th quotient with respect to the canonical filtration - to be precise, we let

$$
\left(\bar{Q}_{\leq l}(V)\right)_{i}= \begin{cases}\bar{Q}_{i}(V), & i \leq l, \\ \bar{Q}_{i}(V) / \operatorname{Ker} d, & i=l+1, \\ 0, & i \geq l+2 .\end{cases}
$$

In the algebra case $V=A$, these are DG algebras. The quotient $\bar{Q}_{\leq 0}(V)$ is quasiisomorphic to $V$. In this Subsection, we will study the first non-trivial quotient $\bar{Q}_{\leq 1}(V)$. It is convenient to consider its quasiisomorphic quotient $V^{b}$. defined by

$$
V_{0}^{\mathrm{b}}=\bar{Q}_{0}(V), \quad V_{1}^{\mathrm{b}}=\bar{Q}_{1}(V) / \operatorname{Im} d .
$$

This is a complex with two terms, in homological degrees 0 and 1 . We will need the following standard piece of linear algebra used for such complexes.

Definition 4.8. Assume given a complex $K_{\bullet}, K_{1} \stackrel{d}{\rightarrow} K_{0}$ in some abelian category $\mathcal{C}$. By a splitting of $K$. we will understand an object $K_{01} \in \mathcal{C}$ equipped with a surjective map $a: K_{01} \rightarrow K_{0}$ and an injective map $b: K_{1} \rightarrow K_{01}$ such that $d=a \circ b, b$ induces an isomorphism Ker $d \rightarrow \operatorname{Ker} a$, and $a$ induces an isomorphism Coker $b \rightarrow$ Coker $d$.

If the category $C$ is symmetric and tensor, and $K$. is a DG algebra in $C$, then we will say that a splitting $\left\langle K_{01}, a, b\right\rangle$ is multiplicative if $K_{01}$ is an algebra in $\mathcal{C}$, $a$ is an algebra map, so that the $K_{0}$-bimodule $K_{1}$ becomes a $K_{01}$-bimodule, and $b$ is a map of $K_{01}$-bimodules.

We note right away that for a multiplicative splitting $\left\langle K_{01}, a, b\right\rangle$ of a DG algebra $K_{\text {. }}$, the complex $K_{1} \stackrel{b}{\rightarrow} K_{01}$ which we denote by $\widetilde{K}$. is also a DG algebra, and $a: K_{01} \rightarrow K_{0}$ induces a surjective DG algebra map $s: \widetilde{K} . \rightarrow K_{\bullet}$; one checks easily that $s$ is a DG splitting of the augmentation map $K_{\bullet} \rightarrow H_{0}\left(K_{\bullet}\right)=$ Coker $d$ in the sense of Subsection 4.1.

In general, a complex $K$. represents by Yoneda a class in the extension group $\operatorname{Ext}^{2}\left(H_{0}\left(K_{\bullet}\right), H_{1}\left(K_{\bullet}\right)\right)$; a splitting exists if and only if this class is trivial. In 
particular, any complex of $k$-vector spaces, including the complex $V_{\bullet}^{b}$, admits a splitting (but not in a way functorial in $V$ ). All splittings of a given complex are isomorphic and form a groupoid $\operatorname{Spl}\left(K_{\bullet}\right)$, possibly an empty one. A quasiisomorphism $s: K_{\text {• }}^{\prime} \rightarrow K$. between two length-2 complexes induces an equivalence $s^{*}: \operatorname{Spl}\left(K_{\bullet}\right) \rightarrow \operatorname{Spl}\left(K_{\bullet}^{\prime}\right)$. Explicitly, for any splitting $K_{01}$ of the complex $K_{\bullet}$, $s^{*} K_{01}$ is the middle homology of the complex

$$
0 \longrightarrow H_{1}\left(K_{\bullet}\right) \stackrel{b \oplus\left(d^{\prime} \circ s\right)}{\longrightarrow} K_{01} \oplus K_{0}^{\prime} \stackrel{a \oplus(-s)}{\longrightarrow} K_{0} \longrightarrow 0 .
$$

We leave it to the reader to check that this is indeed a splitting of $K_{\bullet}^{\prime}$, and that $s^{*}$ is an equivalence. We also leave it to the reader to check that if the category $\mathcal{C}$ is symmetric and tensor, $K_{\text {. and }} K^{\prime}$ are DG algebras, $s: K^{\prime} \rightarrow K_{\bullet}$ is a DG algebra quasiisomorphism, and $K_{01}$ is a multiplicative splitting of $K_{\bullet}$, then $s^{*} K_{01}$ has a natural structure of a multiplicative splitting of $K_{\bullet}^{\prime}$, and $s^{*}$ also identifies multiplicative splittings of $K$. and $K_{\text {. }}^{\prime}$.

Lemma 4.9. For any $k$-vector space $V$, the dual $\left(V_{1}^{b}\right)^{*}$ to the degree-1 term $V_{1}^{b}$ of the complex $V_{\text {. }}^{b}$ is naturally identified with the space of symmetric reduced $k^{*}$ equivariant 2-cocycles of the additive group of $V$ with coefficients in $k$ - in other words, of $k^{*}$-equivariant cocycles $c: V \times V \rightarrow k$ such that in addition to the cocycle condition, we have

$$
c(0, v)=c(v, 0)=0, \quad c\left(v_{1}, v_{2}\right)=c\left(v_{2}, v_{1}\right)
$$

for any $v, v_{1}, v_{2} \in V$.

Proof. Using the explicit form of the cube construction given in the end of Subsection 3.3, we see that $\left(V_{1}^{b}\right)^{*}$ is naturally identified with the space of all maps $c: V \times V \rightarrow k$ such that $c(v, 0)=c(0, v)=0$ for any $v \in V$, and

$$
\begin{aligned}
& c\left(v_{1}, v_{3}\right)+c\left(v_{2}, v_{4}\right)-c\left(v_{1}+v_{2},\right.\left.v_{3}+v_{4}\right)= \\
&=c\left(v_{1}, v_{2}\right)+c\left(v_{3}, v_{4}\right)-c\left(v_{1}+v_{3}, v_{2}+v_{4}\right)
\end{aligned}
$$

for any $v_{1}, v_{2}, v_{3}, v_{4} \in V$. If $v_{1}=v_{4}=0$, this shows that $c$ is a symmetric function. Using this and taking $v_{4}=0$, we get the cocycle condition on $c$. Conversely, if $c$ is a cocycle, then

$$
\begin{aligned}
c\left(v_{1}+v_{2}, v_{3}+v_{4}\right)= & c\left(v_{1}+v_{2}+v_{3}, v_{4}\right)+c\left(v_{1}+v_{2}, v_{3}\right)-c\left(v_{3}, v_{4}\right)= \\
= & c\left(v_{1}+v_{2}+v_{3}, v_{4}\right)+c\left(v_{2}, v_{3}\right)+c\left(v_{1}, v_{2}+v_{3}\right)- \\
& -c\left(v_{1}, v_{2}\right)-c\left(v_{3}, v_{4}\right),
\end{aligned}
$$


and similarly with $v_{2}$ and $v_{3}$ interchanged, so that (4.2) reduces to

$$
c\left(v_{2}, v_{3}\right)=c\left(v_{3}, c_{2}\right),
$$

which means that the cocycle is symmetric.

As a corollary of this Lemma, we see that for any $k$-vector space $V$ we have a completely canonical symmetric $k^{*}$-equivariant cocycle $c: V \times V \rightarrow V_{1}^{b}$, so that the correspoding central extension $V_{01}^{b}$ of $V$ by $V_{1}^{b}$ is an abelian group equipped with an action of $k^{*}$. One checks easily that the image $d(c)$ of this cocycle under the differential $V_{1}^{b} \rightarrow V_{0}^{b}$ in the complex $V^{b}$ is the coboundary of the tautological 1-cochain $V \rightarrow V_{0}^{\mathrm{b}}=\overline{k[V]}_{k^{*}}, v \mapsto[v]$. Therefore we have a natural commutative diagram

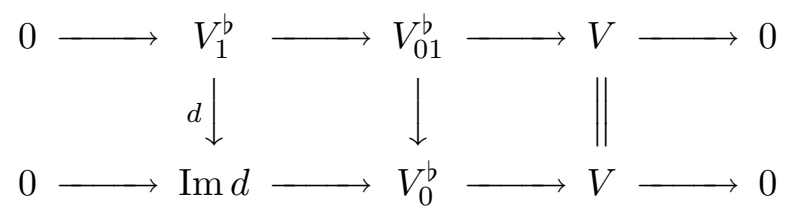

with exact rows, and $V_{01}^{b}$ is canonically a splitting of the complex $V_{\text {. }}^{b}$ in the sense of Definition 4.8. Explicitly, we have $V_{01}^{b}=V_{1}^{b} \times V$ as a set, with addition given by

$$
\left(v_{1} \times v\right)+\left(v_{1}^{\prime} \times v^{\prime}\right)=\left(v_{1}+v_{1}^{\prime}+c\left(v, v^{\prime}\right)\right) \times\left(v+v^{\prime}\right) .
$$

Moreover, for any $k$-vector spaces $V, W$, the multiplication map $\bar{Q} .(V) \otimes \bar{Q} .(W) \rightarrow$ $\bar{Q} .(V \otimes W)$ induces a multiplication map $V_{.}^{b} \otimes W^{b} \rightarrow(V \otimes W)_{.}^{b}$, so that we can define a multiplication map $V_{01}^{b} \times W_{01}^{b} \rightarrow(V \otimes W)_{01}^{b}$ by

$$
\left(v_{1} \times v\right) \cdot\left(w_{1} \times w\right)=\left(v_{1} \cdot d\left(w_{1}\right)\right) \times(v \otimes w) .
$$

We leave it to the reader to check that this multiplication map is bilinear (this amount to checking that $\left.c\left(v, v^{\prime}\right) \otimes w=c\left(v \otimes w, v^{\prime} \otimes w\right)\right)$, and compatible with the projections to $\bar{Q}_{0}(V), \bar{Q}_{0}(W)$. Moreover, since the complex $(V \otimes W)^{b}$. is concentrated in degrees 0 and 1 , we have $v_{1} \cdot d\left(w_{1}\right)-d\left(v_{1}\right) \cdot w_{1}=d\left(v_{1} \cdot w_{1}\right)=0$ for any $v_{1} \in V_{1}^{b}, w_{1} \in W_{1}^{b}$, so that $v_{1} \cdot d\left(w_{1}\right)=d\left(v_{1}\right) \cdot w_{1}$. Therefore the multiplication is also associative, and for an associative $k$-algebra $A, A_{01}^{b}$ becomes an associative algebra and a multiplicative splitting of the DG algebra $A^{b}$.

We conclude that for any $k$-algebra $A$, the DG algebra $A^{b} \rightarrow A$ admits a completely canonical multiplicative spliting $A_{01}^{b}$, so that the augmentation map $A_{\text {. }}^{\text {b }} \rightarrow A$ admits a completely canonical DG splitting. However, this splitting 
cannot be used in Proposition 4.7, since $A_{01}^{b}$ is not an algebra over $k$. Namely, we have the following.

Lemma 4.10. For any $k$-vector space $V, V_{01}^{b}$ has a natural structure of a module over the ring $W_{2}(k)$ of second Witt vectors of the field $k$, and multiplication by $p \in W_{2}(k)$ induces an isomorphism between the quotient $V=V_{01}^{b} / V_{1}^{b}$ and the subspace $H_{1}\left(V_{.}^{b}\right) \subset V_{1}^{b} \subset V_{01}^{b}$. If $A$ is an associative $k$-algebra, then $A_{01}^{b}$ is an algebra over $W_{2}(k)$.

Proof. We note that multiplication by $p$ is already defined for any abelian group, independently of any $W_{2}(k)$-module structure. For $V_{01}^{b}$, the quotient $V_{0}^{b}$ and the subspace $V_{1}^{\mathrm{b}}$ are by definition annihilated by $p$; therefore multiplication by $p$ on $V_{01}^{b}$ must factor through a map $V \rightarrow H_{1}\left(V_{\bullet}^{b}\right) \cong H_{1}(\bar{Q} .(V))$. Since $\bar{Q} .(V)$ is additive in $V$, to prove that this map is an isomorphism, it suffices to consider the 1-dimensional vector space $V=k$. Now, $k_{01}^{b}$ is by definition an algebra and an extension of $k_{0}^{b}$ by $H_{1}(\bar{Q} .(k))=k$. By definition, $k_{0}^{b}=\bar{Q}_{0}(k)=\overline{\operatorname{Span}_{k}}(k)$ is the algebra of $k^{*}$-coinvariants of the algebra of linear combinations of non-zero elements of the field $k$. Since $k^{*}$ acts transitively on the set $k \backslash\{0\}$ of these non-zero elements, the augmentation map $k_{0}^{b} \rightarrow k$ is actually an isomorphism, and $k_{01}^{b}$ is in fact a $k^{*}$-equivariant extension of $k$ by $k$. The ring $W_{2}(k)$ of second Witt vectors is another such extension (the $k^{*}$-action on $W_{2}(k)$ is induced from the multiplicative splitting of the map $W_{2}(k)^{*} \rightarrow k^{*}$ given by the Teichmüller representatives). But the extension $k_{01}^{b}$ is by construction universal. Therefore we have a surjective map $k_{01}^{b} \rightarrow W_{2}(k)$, which must then be an isomorphism for dimension reasons.

Thus $k_{01}^{b} \cong W_{2}(k)$ as rings. In particular, multiplication by $p$ on $k_{01}^{b}$ indeed induces an isomorphism $k=W_{2}(k) / p \cong k \subset W_{2}(k)$. To prove the other claims, we note that since $V$ is a $k$-bimodule, and $A$ is a $k$-algebra, $V_{01}^{b}$ is a $k_{01}^{b}$-bimodule, and $A_{01}^{b}$ is a $k_{01}^{b}$-algebra, by (4.4).

We will use this fact to identify splittings of $V^{b}$ in the category of $k$-vector spaces, and flat liftings of $V$ to $W_{2}(k)$ - that is, flat $W_{2}(k)$-modules $V^{\prime}$ such that $V^{\prime} / p$ is identified with $V$. We use the canonical splitting $V_{01}^{b}$ of $V_{\text {. }}^{\text {. defined over }}$ $W_{2}(k)$, and functoriality of splittings with respect to quasiisomorphisms. We note that pair $\left\langle V^{\prime}, V^{\prime} / p \cong V\right\rangle$ of a flat $W_{2}(k)$-module $V^{\prime}$ and an isomorphism $V^{\prime} / p \cong V$ form a groupoid which we denote by $\operatorname{Lift}(V)$. 
Lemma 4.11. The groupoid $\operatorname{Lift}(V)$ of flat liftings $V^{\prime}$ of a k-vector space $V$ to $W_{2}(k)$ is equivalent to the groupoid $\operatorname{Spl}\left(V_{\bullet}\right)$ of splittings $\left\langle V_{\bullet}, s\right\rangle$ of the complex $V_{\text {. }}^{\text {. }}$ in the category of $k$-vector spaces. If $V=A$ is an associative algebra over $k$, then this equivalence identifies flat liftings $A^{\prime}$ equipped with a structure of an algebra over $W_{2}(k)$ and multiplicative splittings of $A_{\text {. }}^{\text {. }}$.

Proof. Define a length-2 complex $\widetilde{V}$. by $\widetilde{V}_{0}=V_{01}^{b}, \widetilde{V}_{1}=V_{1}^{b} \oplus V, d: \widetilde{V}_{1} \rightarrow \widetilde{V}_{0}$ is the direct sum of the map $b: V_{1}^{b} \rightarrow V_{01}^{b}$ and its restriction to $V \cong H_{1}\left(V_{.}^{b}\right) \subset V_{01}^{b}$. Say that a splitting $\widetilde{V}_{01}$ of this complex in the category of $W_{2}(k)$ is admissible if multiplication by $p$ on $\widetilde{V}_{01}$ factors through the natural isomorphism between the quotient $V \cong H_{0}\left(V_{\bullet}^{b}\right) \cong H_{0}\left(\widetilde{V}_{\bullet}\right)$ of $\widetilde{V}_{01}$ and the subobject $V \subset V \oplus V_{1}^{b}=\widetilde{V}_{1} \subset \widetilde{V}_{01}$.

Define a map $s: \widetilde{V}$. $\rightarrow V_{\text {. }}^{\text {by }}$ the commutative diagram

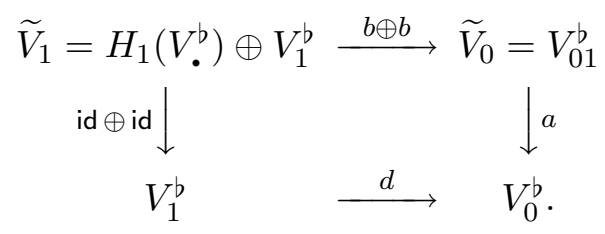

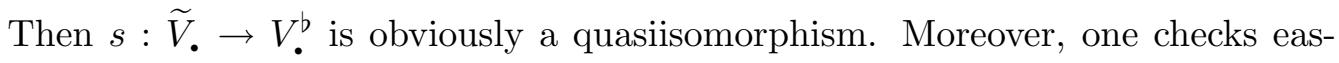
ily that a splitting $V_{01}$ of the complex $V_{01}^{b}$ in the category of $W_{2}(k)$-modules is annihilated by $p$ if and only if $s^{*} V_{01}^{b}$ is admissible.

On the other hand, a lifting $V^{\prime}$ of the $k$-vector space $V$ to $W_{2}(k)$ gives by definition a splitting of the complex $V \stackrel{0}{\rightarrow} V$ with trivial differential, which we denote by $\bar{V}$. . Define a map $s_{1}: \widetilde{V}$. $\rightarrow \bar{V}$. by the commutative diagram

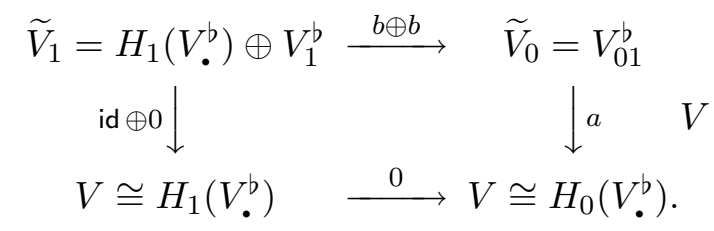

Then this is also a quasiisomorphism, and a splitting $\bar{V}_{01}$ of the complex $\bar{V}$. comes from a lifting $V^{\prime}$ if and only if, again, $s_{1}^{*} \bar{V}_{01}$ is admissible. Thus the equivalences $s^{*}, s_{1}^{*}$ identify the category of liftings $V^{\prime}$ of $V$ to $W_{2}(k)$, the category of splitting of $V^{\text {b }}$ over $k$, and the category of admissible splitting of $\widetilde{V}$. over $W_{2}(k)$. This proves the first claim. To prove the second claim, it suffices to notice that $\widetilde{A}$. is naturally a DG algebra over $W_{2}(k)$, and both $s$ and $s_{1}$ are DG algebra maps. 
Corollary 4.12. To any flat lifting $A^{\prime}$ of an associative $k$-algera $A$ to the ring $W_{2}(k)$ of second Witt vectors of $k$, one can associate in a functorial way a $D G$ splitting $A . \rightarrow \bar{Q}_{\leq 1}(A)$ of the projection $\bar{Q}_{\leq 1}(A) \rightarrow A$.

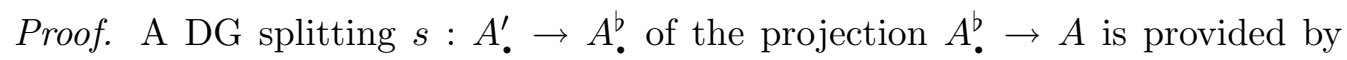
Lema 4.11. For any two DG algebras $B^{1}, B^{2}$ equipped with maps $B^{1} \rightarrow B$, $B^{2} \rightarrow B$ to a DG algebra $B$, we will denote by $B^{1} \oplus_{B} B^{2}$ the subalgebra in $B_{1} \oplus B_{2}$ consisting of elements with the same image in $B$ - in other words, the kernel of the difference $B_{1} \oplus B_{2} \rightarrow B$ of the given maps. We note that if $B_{1} \rightarrow B$ is a surjective map with an acyclic kernel, then the natural projections $B_{1} \oplus_{B} B_{2} \rightarrow B_{2}$ also is a surjective DG algebra map with an acyclic kernel. In particular, $A_{\bullet}=A_{\bullet}^{\prime} \oplus_{A_{\bullet}^{b}} \bar{Q}_{\leq 1}(A)$ is quasiisomorphic to $A_{\bullet}^{\prime}$, hence to $A$, and the projection $A . \rightarrow \bar{Q}_{\leq 1}(A)$ is a DG splitting of $\bar{Q}_{\leq 1}(A) \rightarrow A$.

Remark 4.13. From the topological point of view, Lemma 4.11 is in a sense completely obvious. We note that the cube construction in its explicit form given the end of Subsection 3.3 has a straightforward generalization to modules $V$ over the Witt vectors ring $W(k)$ - indeed, the $k$-linear span $\operatorname{Span}_{k}(V)$ is defined for any $W(k)$-module just as well as for a $k$-vector space, and the Teichmiller representatives give canonical map $k^{*} \rightarrow W(k)^{*}$, so that the quotient $\overline{\operatorname{Span}_{k}}(V)$ also makes sense. Thus for any $W(k)$-algebra $A^{\prime}$, we have a DG algebra $\bar{Q} .\left(A^{\prime}\right)$ over $k$ which computes the $k^{*}$-invariant part of the homology $H(k)\left(A^{\prime}\right)$ of the spectrum $A^{\prime}$ with cofficients in $k$. Once we are given a lifting $A^{\prime}$, as in Corollary 4.12 , we can consider the DG algebra $\bar{Q}_{\leq 1}\left(A^{\prime}\right)$ and the natural map $\bar{Q}_{\leq 1}\left(A^{\prime}\right) \rightarrow \bar{Q}_{\leq 1}(A)$. However, the generator of the group $H_{1}\left(\mathrm{St}_{.}^{k^{*}}(k)\right)=k$ is the Bokstein homomorphism, which controls precisely the liftings of the coefficients of cohomology from $k$ to $W_{2}(k)$. Because of this, both the 0 -th and the 1-st homology groups of the complex $\bar{Q}_{\leq 1}\left(A^{\prime}\right)$ are isomorphic to $A$, not to $A^{\prime}$, and the map $\bar{Q}_{\leq 1}\left(A^{\prime}\right) \rightarrow \bar{Q}_{\leq 1}(A)$ is trivial on homology in degree 1 , so that it factors through the quotient $\bar{Q}_{\leq 1}\left(A^{\prime}\right) /(A[1])$. The natural augmentation map $\bar{Q}_{\leq 1}\left(A^{\prime}\right) /(A[1]) \rightarrow A$ is then a quasiisomorphism, and the map $\bar{Q}_{\leq 1}\left(A^{\prime}\right) /(A[1]) \rightarrow \bar{Q}_{\leq 1}(A)$ is a DG splitting of the projection $\bar{Q}_{\leq 1}(A) \rightarrow A$. This is the same DG splitting as in Corollary 4.12. Instead of making precise the procedure sketched above, we found it more convenient to do things in a more explicit way. 
4.3. Splitting in higher orders. Unfortunately, we could not find a statement analogous to Corollary 4.12 for the splitting of the projection $\bar{Q} .(A) \rightarrow A$ in higher orders. From the topological point of view, it would be enough to require that $A$ lifts to a ring spectum - that is, there exists a ring spectrum $\widetilde{A}$ such that $A=H(k)(\widetilde{A})$, the homology of the spectrum $\widetilde{A}$ with coefficients in $k$. However, this is not easy to verify in practice. Therefore in higher orders, we have to rely on dimension reasons to insure that a splitting exists. We now recall the necessary generalities from deformation theory.

Assume given a flat associative algebra $A$ in an abelian symmetric tensor category $\mathcal{C}$, and an $A$-bimodule $M \in A$-bimod which is flat as an object in $\mathcal{C}$. By a square-zero extension of $A$ by $M$ we will understand a flat associative algebra $\widetilde{A}$ in $C$ equipped with an algebra map $\widetilde{A} \rightarrow A$ and an embedding $M \rightarrow \widetilde{A}$ such that the sequence

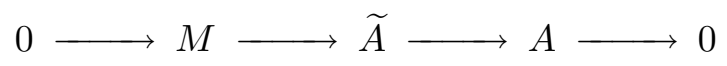

is exact, and the induced $\widetilde{A}$-bimodule structure on the two-sided ideal $M \subset \widetilde{A}$ is induced from the given $A$-bimodule structure via the algebra map $\widetilde{A} \rightarrow A$. If $\mathcal{C}$ is the category of vector spaces, then by a standard construction, all such square-zero extensions are classified by elements in the Hochschild cohomology group $H H^{2}(A)$. Let us recall the details of this construction, so that the reader will see that it works equally well for an arbitraty $\mathcal{C}$.

Assume given an object $\widetilde{A} \in \mathcal{C}$ which is an extension of the object $A$ by the object $M$. By definition, for any structure of a square-zero extension on $\widetilde{A}$, the multiplication map $\widetilde{A}^{\otimes 2} \rightarrow \widetilde{A}$ must factor through the quotient $\widetilde{A}^{\otimes 2} / M^{\otimes 2}$. This quotient is an extension of $A^{\otimes 2}$ by $(A \otimes M) \oplus(M \otimes A)$. Using the $A$-bimodule structure on $M$, we can apply the multiplication maps $A \otimes M \rightarrow M, M \otimes A \rightarrow M$, and reduce this extension to an extension of $A^{\otimes 2}$ by $M \oplus M$, which we denote by $\overline{A^{\otimes 2}}$. We have a natural map $\widetilde{A}^{\otimes 2} \rightarrow \overline{A^{\otimes 2}}$, and for any structure of a squre-zero extension on $\widetilde{A}$, the multiplication must factor through a map $\overline{A^{\otimes 2}} \rightarrow \widetilde{A}$.

However, and this is the crucial part of the construction, the extension $\overline{A^{\otimes 2}}$ can be also obtained "in a linear way", without using the tensor product $\widetilde{A}^{\otimes 2}$. Namely, we note that for any $X \in \mathcal{C}$, we tautologically have

$$
\mathcal{E}_{\mathrm{x}_{\mathcal{C}}}(X, M) \cong \mathcal{E}_{\mathrm{x}_{A \text {-bimod }}(A \otimes X \otimes A, M)}
$$


where $\mathcal{E} \mathrm{x}(-,-)$ denotes the groupoid of extensions, and in the right-hand side, we consider extensions in the category of $A$-bimodules in $\mathcal{C}$. In particular, $\widetilde{A} \in$ $\mathcal{E} \mathrm{x}_{\mathcal{C}}(A, M)$ corresponds to an object $\bar{A} \in \mathcal{E} \mathrm{x}_{A \text {-bimod }}(A \otimes A \otimes A, M)$. Unwinding the definitions, we immediately see that the object $\overline{A^{\otimes 2}} \in \mathcal{E}_{\mathrm{x}_{\mathcal{C}}}\left(A^{\otimes 2}, M \oplus M\right)$ corresponds to the object $d_{1}^{*} \bar{A} \oplus d_{3}^{*} \bar{A}$ in

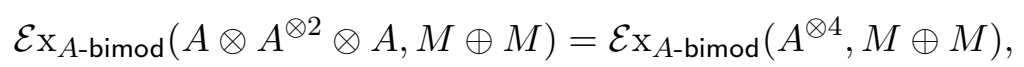

where $d_{i}: A^{\otimes 4} \rightarrow A^{\otimes 3}, i=1,2,3$, is obtained by applying the multiplication map $A^{\otimes 2} \rightarrow A$ at the $i$-th tensor sign in $A^{\otimes 4}=A \otimes A \otimes A \otimes A$.

Now, to define a structure of a square-zero extension on $\widetilde{A}$, we have to define a multiplication map $\overline{A^{\otimes 2}} \rightarrow \widetilde{A}$ which fits into a commutative diagram

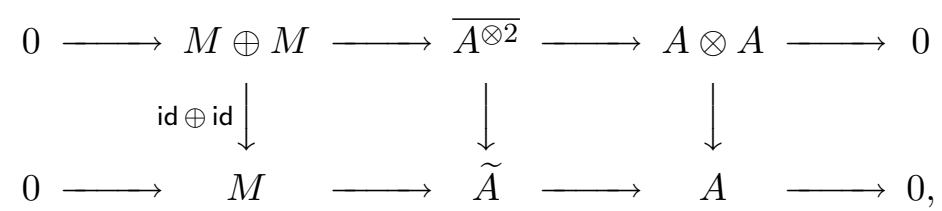

where on the right we have the multiplication map in $A$. In terms of the identification (4.5), this is equivalent to giving a splitting of the extension $\delta_{3}^{*} \bar{A} \in$

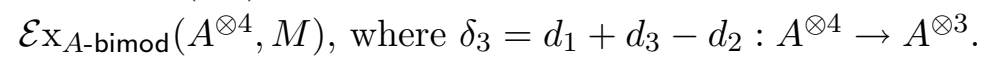

To sum up: giving an extension $\widetilde{A}$ of $A$ by $M$ equipped with a square-zero multiplication is equivalent to giving an extension $\bar{A}$ of the $A$-bimodule $A^{\otimes 3}$ by $M$, and a map $\bar{\delta}: A^{\otimes 4} \rightarrow \bar{A}$ whose composition with the natural projection is equal to $\delta_{3}: A^{\otimes 4} \rightarrow A^{\otimes 3}$.

The map $\delta_{3}$ is of course a part of the standard bar resolution $C .(A)=\left\langle A^{\otimes^{\bullet}}, \delta_{\bullet}\right\rangle$ of the diagonal bimodule $A \in A$-bimod; we leave it to the reader to check that the multiplication on $\widetilde{A}$ is associative if and only if the composition $\bar{\delta} \circ \delta_{4}: A^{\otimes 5} \rightarrow \bar{A}$ is equal to 0 . In other words, square-zero extensions are classified by an extension

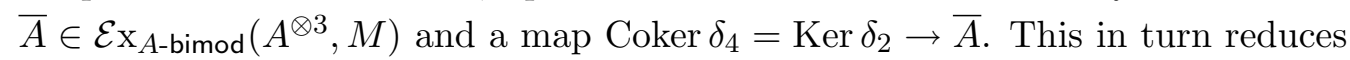
to an extension of Coker $\delta_{3}=\operatorname{Ker} \delta_{1}$ by $M$.

By definition, $\delta_{1}: A \otimes A \rightarrow A$ is the multiplication map in the algebra $A$; we denote its kernel by $\mathcal{I}_{A} \subset A^{\otimes 2}\left(\mathcal{I}_{A}\right.$ is sometimes known as the bimodule of non-commutative differential forms).

Definition 4.14. Reduced Hochschild cohomology of a flat associative algebra $A \in \mathcal{C}$ with coefficients in $M \in A$-bimod is given by

$$
\overline{H H}^{\bullet+1}(A, M)=\operatorname{Ext}_{A \text {-bimod }}\left(\mathcal{I}_{A}, M\right) .
$$


The reduced Hochschild cohomology $\overline{H H}^{\bullet}(A)$ is the reduced Hochschild cohomology of $A$ with coefficients in the diagonal bimodule $A$.

We conclude that square-zero extensions $\widetilde{A}$ of $A$ by $M$ are classified, up to an isomorphism, by elements in the reduced Hochschild cohomology group $\overline{H H}^{2}(A, M)$.

Remark 4.15. As in Subsection 4.2, we have chosen to do things in a very explicit way. In a more abstract approach, the linearization procedure described above shows that square-zero extensions of an assocative algebra $A$ by a bimodule $M$ are classified by elements in the extension group $\operatorname{Ext}^{1}(C$. $(A), M[1])$; the group is computed in the category $\operatorname{Fun}\left(\Delta^{o}, A\right.$-bimod) of simplicial $A$-bimodules, $C$. $(A)$ is the bar resolution of $A$ considered as a simplicial object, and $M[1]$ is the simplicial object corresponding to the shift of $M$ via the Dold-Kan equivalence. One can also develop a version for commutative algebras, in which $\Delta^{o}$ is replaced with the category $\Gamma_{+}$of finite pointed sets, as in Subsection 3.2, and $M[1]$ is replaced with $M \otimes T^{*}$. In characteristic 0 , this gives an invariant definition of the Harrison cohomology and the cotangent complex; in general, as we have seen in Subsection 3.2, the resulting Ext ${ }^{\circ}$-groups also involve the Steenrod algebra.

Now, the category of complexes of $k$-vector spaces is a symmetric tensor abelian category, so that all the above can be applied to DG algebras. However, to study DG splittings, we need a version of the formalism which works "up to a quasiisomorphism". We will actually need even more generality. We assume given an abelian category $\mathcal{C}$ and a thick triangulated subcategory

$$
\operatorname{Ac} \subset \mathcal{D}^{b}(\mathcal{C})
$$

of "negligible objects" in its derived category $\mathcal{D}^{b}(\mathcal{C})$. We will say that a map

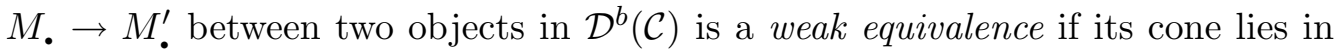
Ac $\subset \mathcal{D}^{b}(\mathcal{C})$. The category $\mathcal{C}$ is of course embedded into $\mathcal{D}^{b}(\mathcal{C})$, and the class of surjective weak equivalences between objects in $\mathcal{C}$ is localizing - one can develop the calculus of fractions and define the quotient category $\mathrm{Ho}(\mathcal{C})$. Objects in $\mathrm{Ho}(\mathcal{C})$ are the same as in $\mathcal{C}$, and a map between $M, M^{\prime} \in \mathrm{Ho}(\mathcal{C})$ is given by an equivalence class of diagrams

$$
M \longleftarrow \widetilde{M} \longrightarrow M^{\prime}
$$


in $\mathcal{C}$, where the left arrow is a weak equivalence. We obviously have a natural functor $\mathrm{Ho}(\mathcal{C})$ to the quotient category $\mathcal{D}^{b}(\mathcal{C}) / \mathrm{Ac}$. We will assume that

for any object $M \in \mathcal{C}$, there exist a surjection $P \rightarrow A$ and an injection $M \rightarrow Q$ with $I, Q \in \operatorname{Ac} \cap \mathcal{C}$.

Lemma 4.16. Under the assumption 4.8, the natural functor $\mathrm{Ho}(\mathcal{C}) \rightarrow \mathcal{D}^{b}(\mathcal{C}) / \mathrm{Ac}$ is an equivalence of categories.

Proof. To prove that the functor is essentially surjective, take some object $M \in$ $\mathcal{D}^{b}(\mathcal{C})$, assume that $M \in \mathcal{D}^{\leq n}(\mathcal{C}) \cap \mathcal{D}^{\geq n^{\prime}}(\mathcal{C})$ for some $n>n^{\prime}$, and, using the standard $t$-structure on $\mathcal{D}^{b}(\mathcal{C})$, decompose it into an exact triangle

$$
M_{1} \longrightarrow M \longrightarrow M_{0}[-n] \stackrel{\delta}{\longrightarrow}
$$

with $M_{1} \in \mathcal{D}^{\leq n-1}(\mathcal{C}) \cap \mathcal{D}^{\geq n^{\prime}}, M_{0} \in \mathcal{C}$. Choose a surjection $\tau: \widetilde{M} \rightarrow M_{0}$ such that $\tau \circ \delta=0$, and choose a surjection $\tau^{\prime}: P \rightarrow \widetilde{M}$ with $P \in$ Ac $\cap \mathcal{C}$. Let $M_{0}^{\prime}$ be the kernel of the surjection $\tau^{\prime} \circ \tau: P \rightarrow M_{0}$, and let $\iota: M_{0} \rightarrow M_{0}^{\prime}[1]$ be the corresponding extension class. Then $\tau^{\prime} \circ \tau \circ \delta=0$, so that $\delta$ factors as $\delta=\delta^{\prime} \circ \iota$ with some $\delta^{\prime}: M_{0}^{\prime}[n-1] \rightarrow M_{1}[1]$, and we have a triangle

$$
M \stackrel{\iota^{\prime}}{\longrightarrow} M^{\prime} \longrightarrow P[n-1] \longrightarrow,
$$

where $M^{\prime}$ is the cone of the map $\delta^{\prime}$. Then by construction, $\iota^{\prime}: M \rightarrow M^{\prime}$ is a weak equivalence, and $M^{\prime} \in \mathcal{D}^{\leq n-1}(\mathcal{C}) \cap \mathcal{D}^{\geq n^{\prime}}(\mathcal{C})$.

By the dual argument using injections instead of surjections, every $M \in$ $\mathcal{D}^{\leq n}(\mathcal{C}) \cap \mathcal{D}^{\geq n^{\prime}}(\mathcal{C})$ admits a weak equivalence $M^{\prime} \rightarrow M$ with $M^{\prime} \in \mathcal{D}^{\leq n} \cap \mathcal{D}^{\geq n^{\prime}}$. Then by induction, every $M \in \mathcal{D}^{b}(\mathcal{C})$ is weakly equivalent to some $M^{\prime} \in \mathcal{C}=$ $\mathcal{D}^{\geq 0}(\mathcal{C}) \cap \mathcal{D}^{\leq 0}(\mathcal{C})$, so that the comparison functor $\mathrm{Ho}(\mathcal{C}) \rightarrow \mathcal{D}^{b}(\mathcal{C}) /$ Ac is indeed essentially surjective.

To prove that it is an equivalence, it remains to prove that it is fully faithful that is, for any $M, M^{\prime} \in \mathcal{C}$, any diagram (4.7) with $\widetilde{M} \in \mathcal{D}^{b}(\mathcal{C})$ is equivalent to a diagram with $\widetilde{M} \in \mathrm{Ac} \cap \mathcal{C}$ and a surjective weak equivalence $\widetilde{M} \rightarrow M$. Choosing a weak equivalence $\widetilde{M^{\prime}} \rightarrow \widetilde{M}$ with $\widetilde{M}^{\prime} \in \mathcal{D}^{\geq 0}(\mathcal{C})$ and replacing $\widetilde{M}$ with $\widetilde{M^{\prime}}$, we insure that $\widetilde{M} \in \mathcal{D}^{b}(\mathcal{C})$. To cut it down to an object in $\mathcal{C}$, we argue as above: $\widetilde{M} \in \mathcal{D}^{\leq n}(\mathcal{C})$ for some $n$, and we can construct a map $\tau: P[-n] \rightarrow \widetilde{M}$ with $P \in \mathrm{Ac} \cap \mathcal{C}$ such that $\tau$ is surjective on $n$-th cohomology. Then the composition map $P[-n] \rightarrow M \oplus M^{\prime}$ can be annihilated by composing with some surjection 
$P^{\prime} \rightarrow P$, and by 4.8 , we can insure that $P^{\prime} \in \mathrm{Ac} \cap \mathcal{C}$. We replace $\widetilde{M}$ with the cone of the map $P^{\prime} \rightarrow M$ which lies in $\mathcal{D}^{\leq n-1}(\mathcal{C})$, and proceed by induction.

Thus if the condition 4.8 is satisfied, the category $\mathrm{Ho}(\mathcal{C})$ is triangulated. The basic example of the situation is $\mathcal{C}=C^{b}\left(\mathcal{C}_{0}\right)$, the category of bounded complexes in some abelian category $\mathcal{C}_{0}$, and $\mathrm{Ac} \in \mathcal{D}^{b}(\mathcal{C})$ is the kernel of the natural functor $\mathcal{D}^{b}(\mathcal{C}) \rightarrow \mathcal{D}^{b}\left(\mathcal{C}_{0}\right)$ which sends a complex of complexes in $\mathcal{C}_{0}$ to its total complex. In this case, $\operatorname{Ho}(\mathcal{C})=\mathcal{D}^{b}\left(\mathcal{C}_{0}\right)$. More complicated examples will be used in Section 5 .

Assume now that the abelian category $\mathcal{C}$ is a symmetric tensor category, with the unit object $I \in \mathcal{C}$. Assume also that $A c \subset \mathcal{D}^{b}(\mathcal{C})$ is an ideal with respect to the tensor structure, in the sense that for any $M^{\bullet} \in \mathcal{D}^{b}(\mathcal{C})$ and a flat $P \in \mathrm{Ac} \cap \mathcal{C}$, we have $P \stackrel{L}{\otimes} M^{\bullet} \in$ Ac. Then the simplest way to insure 4.8 is to require the following:

there exist flat objects $Q, P \in \operatorname{Ac} \cap \mathcal{C}$, a surjection $P \rightarrow I$, and an injection $I \rightarrow Q$.

Moreover, assume given a flat algebra $\mathcal{A}$ in $\mathcal{C}$, let $\mathcal{A}$-bimod be the abelian category of $\mathcal{A}$-bimodules in $\mathcal{C}$, and let $\operatorname{Ac}(\mathcal{A}) \subset \mathcal{D}^{b}(\mathcal{A}$-bimod) be the subcategory of objects whose underlying complex lies in $\operatorname{Ac} \subset \mathcal{D}^{b}(\mathcal{C})$. Then the pair $\langle\mathcal{A}$-bimod, $\operatorname{Ac}(\mathcal{A})\rangle$ also satisfies 4.8 . For any $M, M^{\prime} \in \mathcal{A}$-bimod, we denote by

$$
\operatorname{Ext}_{\mathcal{D}}^{n}\left(M, M^{\prime}\right)=\operatorname{Hom}_{\text {Ho }(\mathcal{A} \text {-bimod })}\left(M, M^{\prime}[n]\right), \quad n \geq 0
$$

the graded space of maps between $M$ and $M^{\prime}$ in the quotient category $\mathrm{Ho}(\mathcal{A}$-bimod $)$ $=\mathcal{D}^{b}(\mathcal{A}$-bimod $) / \operatorname{Ac}(\mathcal{A})$. Replacing Ext -groups in the right-hand side of $(4.6)$ with Ext $_{\mathcal{D}}$, we obtain a version of the Hochschild cohomology groups which we call the quotient Hochschild cohomology groups and denote by ${\overline{H H_{\mathcal{D}}}}_{\mathcal{D}}(\mathcal{A}, M)$. We have a natural map $\overline{H H}^{\bullet}(\mathcal{A}, M) \rightarrow{\overline{H H_{\mathcal{D}}}}_{\mathcal{D}}(\mathcal{A}, M)$ (induced by passing to the quotient category). For any map $f: \mathcal{A} \rightarrow \mathcal{A}^{\prime}$ of algebras in $\mathcal{C}$, and any $\mathcal{A}^{\prime}$-bimodule $M$, we have a natural map

$$
f^{*}: \overline{H H}_{\mathcal{D}}^{\bullet}\left(\mathcal{A}^{\prime}, M\right) \rightarrow{\overline{H H_{\mathcal{D}}}}_{{ }_{\mathcal{D}}}(\mathcal{A}, M),
$$

where on the right, $M$ is considered as an $\mathcal{A}$-bimodule via the map $f$. If $f$ is a weak equivalence, then $f^{*}$ is an isomorphism.

Any square-zero extension $\widetilde{\mathcal{A}}$ of an algebra $\mathcal{A}$ in $\mathcal{C}$ by an $\mathcal{A}$-bimodule $M$ gives rise to a class $\Theta(\widetilde{\mathcal{A}}) \in \overline{H H}^{2}(\mathcal{A}, M)$; we denote by $\Theta_{\mathcal{D}}(\widetilde{\mathcal{A}})$ its image in $\overline{H H}_{\mathcal{D}}^{2}(\mathcal{A}, M)$. By a weak splitting of a surjective map $\widetilde{\mathcal{A}} \rightarrow \mathcal{A}$ of algebras in $\mathcal{C}$ we 
will understand a pair of an algebra $\overline{\mathcal{A}}$ in $\mathcal{C}$ and a map $s: \overline{\mathcal{A}} \rightarrow \widetilde{\mathcal{A}}$ such that the composition

$$
\overline{\mathcal{A}} \stackrel{s}{\longrightarrow} \widetilde{\mathcal{A}} \longrightarrow \mathcal{A}
$$

is a surjective weak equivalence. If $\mathcal{C}=C^{b}\left(C_{0}\right)$ for some tensor abelian category $\mathcal{C}_{0}$, so that $\mathcal{A}$ is a DG algebra, then a weak splitting is the same as a DG splitting in the sense of Subsection 4.1.

Lemma 4.17. A square-zero extension $\widetilde{\mathcal{A}} \rightarrow \mathcal{A}$ of an algebra $\mathcal{A}$ in $\mathcal{C}$ by an $\mathcal{A}$ bimodule $M$ admits a weak splitting $s: \overline{\mathcal{A}} \rightarrow \widetilde{\mathcal{A}}$ if and only if its class $\Theta_{\mathcal{D}}(\widetilde{\mathcal{A}}) \in$ $\overline{H H}_{\mathcal{D}}^{2}(\mathcal{A}, M)$ is equal to zero.

Proof. If a weak splitting $s: \overline{\mathcal{A}} \rightarrow \widetilde{\mathcal{A}}$ exists, then the weak equivalence $\sigma: \overline{\mathcal{A}} \rightarrow \mathcal{A}$ induces by $s$ factors through the projection $\widetilde{\mathcal{A}} \rightarrow \mathcal{A}$; therefore the induced extension $\overline{\mathcal{A}} \oplus_{\mathcal{A}} \widetilde{\mathcal{A}}$ splits, so that $\sigma^{*} \Theta_{\mathcal{D}}(\widetilde{\mathcal{A}})=0$. Since $\sigma$, being a weak equivalence, induces an isomorphism of quotient Hochschild cohomology groups, this is equivalent to $\Theta_{\mathcal{D}}(\widetilde{\mathcal{A}})=0$.

Conversely, denote by $\bar{M} \in \mathcal{E}_{\mathrm{x}_{\mathcal{A} \text {-bimod }}}\left(\mathcal{I}_{\mathcal{A}}, M\right)$ the extension corresponding to the square-zero extension $\widetilde{\mathcal{A}}$. If $\Theta_{\mathcal{D}}(\widetilde{\mathcal{A}})=0$, then by definition, the exact triangle

$$
M \longrightarrow \bar{M} \longrightarrow \mathcal{I}_{\mathcal{A}} \longrightarrow
$$

splits in $\mathrm{Ho}(\mathcal{A}$-bimod $)$; explictly, this means that there exists an $\mathcal{A}$-bimodule $\widetilde{M} \in \mathcal{A}$-bimod and a map $\tau: \widetilde{M} \rightarrow \bar{M}$ such that the induced map $\widetilde{M} \rightarrow \mathcal{I}_{\mathcal{A}}$ is a surjective weak equivalence. Thus $\widetilde{M}$ is an extension of $\mathcal{I}_{\mathcal{A}}$ by some $\widetilde{M}^{\prime} \in \operatorname{Ac}(\mathcal{A})$, and the extension $\bar{M}$ is induced from $\widetilde{M}$ by means of the map $\tau: \widetilde{M}^{\prime} \rightarrow M$. Let $\overline{\mathcal{A}}$ be the square-zero extension of $\mathcal{A}$ by $\widetilde{M}^{\prime}$ corresponding to $\widetilde{M}$; then the projection $\overline{\mathcal{A}} \rightarrow \mathcal{A}$ is a surjective weak equivalence, and $\tau$ induces an algebra map $s: \overline{\mathcal{A}} \rightarrow \widetilde{\mathcal{A}}$ compatible with the projections to $\mathcal{A}$.

Applying Lemma 4.17 by induction, it is not difficult to show that for any $k$-algebra $A$ such that $A$-bimod has finite homological dimension and moreover, $\overline{H H}^{i}(A)=0$ for $i \geq 2 p$, any DG splitting of the projection $\bar{Q}_{\leq 1}(A) \rightarrow A$ extends to a DG splitting of the projection $\bar{Q}$. $(A) \rightarrow A$; if in addition $A$ can be lifted by a flat algebra over $W_{2}(k)$, this means that $A$ admits a Cartier isomorphism $H H_{\text {. }}\left(A^{(1)}\right)((u)) \cong H P .(A)$. We do not do this, since we will prove a more general statement in the next section. For now, let us remark the following. In 
the commutative case [DI], there are two conditions that insure the existence of a global Cartier isomorphism:

(i) the variety $X / k$ must be liftable to $W_{2}(k)$, and

(ii) we must have $p>\operatorname{dim} X$.

The second condition looks like an artifact of the definition, and one might expect that it can be dropped in the general associative case. However, as we see, this is not so: even in the general case, both conditions survive.

\section{Degeneration.}

5.1. Statements. We now turn to our main subject, the degeneration of the Hodge-to-de Rham spectral sequence (1.17).

A degeneration theorem can be stated and proved in the framework of the last section, that is, for an associative algebra $A$ over a field $K$ of characteristic 0 . But this is almost meaningless: very few algebras satisfy the natural sufficient conditions for such degeneration. To get a useful statement, one has to enhance the class of algebras in some way. There are two possibilities for this.

The first one is very well adapted to our method, namely, to the use of the Cartier isomorphism. Instead of an algebra $A$ over a field $K$ of characteristic 0 , one considers a small category $C$ and an associative algebra $\mathcal{A} \in \operatorname{Fun}(C, K)$. To cover more examples, one can also allow some Grothendieck topology $J$ on $C^{\circ}$, so that $\mathcal{A} \in \operatorname{Fun}(C, K)$ is a sheaf of associative algebras with respect to $J$. This is the approach used in $[\mathrm{Ka}]$.

In this paper, we prefer to use the second possibility, which is closer to the way the degeneration conjecture has been formulated in [KS]. One uses the language of $A_{\infty}$-algebras, as in [KS], or the equivalent and more classic language of DG algebras. Lately, DG algebras were studied extensively by B. Toën; we mostly follow his approach (we refer the reader in particular to [TV], joint with M. Vaquié, and also to an excellent overview of the subject given by B. Keller [Ke]).

Let us recall the main definitions. By a perfect complex of modules over a ring or a DG ring $R$. we mean a complex of $R$.-modules quasiisomorphic to a direct summand of a finite complex of finitely generated free (DG) $R_{0}$-modules (in other words, a compact object in the derived category $\mathcal{D}\left(R_{\bullet}\right)$, see [TV]). 
Definition 5.1. Assume given an associative DG algebra $A$. over a commutative $\operatorname{ring} R$.

(i) The algebra $A_{0}$ is called compact if $A$. is a perfect complex of $R$-modules.

(ii) The algebra $A_{0}$ is called smooth if $A_{0}$ is a perfect complex of $A_{0}$-bimodules.

(iii) The algebra $A_{\text {. }}$ is called saturated if it is smooth and compact.

As we have noted in Subsection 2.1, the $\sharp$-construction makes sense for algebras in any symmetric monoidal category; in particular, it works for DG algebras for any DG algebra $A^{\bullet}$ over a field $K$, we have a complex $A_{\sharp}^{\bullet}$ in $\operatorname{Fun}(\Lambda, K)$. We take the corresponding object in $\mathcal{D}(\Lambda, K)$, and define

$$
H H_{\bullet}\left(A^{\bullet}\right)=H H_{\bullet}\left(A_{\sharp}^{\bullet}\right), \quad H C .\left(A^{\bullet}\right)=H C .\left(A_{\sharp}^{\bullet}\right) .
$$

In this language, the degeneration conjecture of Kontsevich and Soibelman can be stated as follows.

Conjecture 5.2 (Kontsevich-Soibelman). For any saturated $D G$ algebra $A^{\bullet}$ over a field $K$ of characteristic 0 , the Hodge-to-de Rham spectral sequence

$$
H H_{\bullet}\left(A^{\bullet}\right)\left[u^{-1}\right] \Rightarrow H C .\left(A^{\bullet}\right)
$$

of (1.17) degenerates at first term, so that $H C$. $\left(A^{\bullet}\right) \cong H H_{\bullet}\left(A^{\bullet}\right)\left[u^{-1}\right]$.

Remark 5.3. It is known that for any smooth projective algebraic variety $X$ over a field $k$, the derived category $\mathcal{D}_{c}(X)$ of coherent sheaves on $X$ is equivalent to the derived category of DG modules over a saturated DG algebra $A^{\circ}$. Moreover, for such an $A^{\bullet}$, the Hochschild homology $H H_{\bullet}\left(A^{*}\right)$ is naturally identified with the Hodge homology $\bigoplus_{i} H^{i+\bullet}\left(X, \Omega_{X}^{i}\right)$ of the variety $X$, the periodic cyclic homology $H P .(A)$ is isomorphic to the de Rham cohomology of $X$, and the Hodge-to-de Rham spectral sequence reduces to the usual Hodge-to-de Rham Spectral sequence $H^{\bullet}\left(X, \Omega^{\bullet}\right) \Rightarrow H_{D R}^{\bullet}(X)$. Thus Kontsevich-Soibelman Conjecture includes the usual Hodge-to-de Rham degeneration.

Our approach to this conjecture - actually, the approach of [DI - is to use reduction to positive characteristic, and herein lies a difficulty. In the commutative case, one usually requires right from the start that all algebras are Noetherian, or better yet, of finite type; this guarantees that an algebra $A$ over a field $K$ is actually defined over a subring $R \subset K$ of finite type over $\mathbb{Z}$. In the general associative case, the Noetherian assumption is very restrictive and breaks easily 
under the most innocuous operations; in particular, a finitely generated algebra needs not be Noetherian. Fortunately, B. Toën has recently proved that saturated DG algebras are in fact "defined by a finite amount of data", so that an algebra over $K$ does admit a model over finitely generated $R \subset K$.

Theorem $5.4([\mathrm{~T}])$. Assume given a saturated $D G$ algebra $A^{\bullet}$ over a ring $R$, and assume that $R=\lim R_{i}$ is the filtered colimit of its subrings $R_{i} \subset R$. Then there exists a saturated $\overrightarrow{D G}$ algebra $A_{i}^{*}$ over one of these subrings $R_{i}$ such that $A^{\bullet} \cong A_{i}^{\bullet} \otimes_{R_{i}} R$.

Here, of course, the tensor product should be understood in the derived sense. Informally speaking, "the collection of saturated DG algebras over $R$ is finitely presented as a functor of $R$ ". We do not know any analog of this statement for sheaves of algebras over a site.

Using Theorem 5.4, we can prove the following.

Theorem 5.5. Assume given a saturated $D G$ algebra $A^{\bullet}$ over a field $K$ of characteristic 0. Assume in addition that $A_{K}^{i}=0$ for $i<0$. Then the Hodge-to-de Rham spectral sequence

$$
H H_{\bullet}\left(A^{\bullet}\right)\left[u^{-1}\right] \Rightarrow H C .\left(A^{\bullet}\right)
$$

of (1.17) degenerates at first term.

This differs from Conjecture 5.2 in one important respect: we require that our DG algebras are concentrated in non-negative degrees. The reason for this is our method, which does not work at all for DG algebras (essentially because Lemma 2.3 has no graded version - the degree of $v^{\otimes p}$ is equal to the degree of $v$ only if both degrees are 0 ). Thus we have to pass to cosimplicial algebras by the Dold-Kan equivalence, and this works only for algebras which are concentrated in non-negative degrees. There are methods for circumventing this difficulty, but at present, all the approaches known to the author are rather complicated and ad hoc. Instead of giving a crumpled exposition, we prefer to postpone the question and give a detailed treatment in a separate paper. We note that a DG algebra which corresponds to a projective algebraic variety $X$ as in Remark 5.3 can be chosen to satisfy the non-negativity assumption. 
5.2. Cosimplicial algebras and splittings. We start by establishing some facts on the relation between DG algebras and comsimplicial algebras (that is, associative algebras in $\operatorname{Fun}(\Delta, k))$. Recall that for any commutative ring $R$, we have the Dold-Kan equivalence $\mathrm{D}: \operatorname{Fun}(\Delta, R \bmod ) \rightarrow C^{\geq 0}(R \bmod )$. While it does not commute with the natural tensor products in both categories, there exists a functorial map

$$
\mathrm{D}\left(V \otimes_{R} W\right)^{\bullet} \rightarrow \mathrm{D}(V)^{\bullet} \otimes_{R} \mathrm{D}(W)^{\bullet},
$$

and this map is compatible with the associativity and commutativity morphisms. If $V^{\bullet}, W^{\bullet} \in \operatorname{Fun}(\Delta, R \bmod )$ are flat over $R$, then this map is a quasiisomorphism. Thus for any DG algebra $A_{R}^{*}$ over $R$ which is concentrated in non-negative degrees, the cosimplicial $R$-module $\mathcal{A}_{R}=\mathrm{D}^{-1}\left(A_{R}^{*}\right) \in \operatorname{Fun}(\Delta, R)$ has a natural structure of an associative algebra over $R$. This gives a functor from the category of DG $R$-algebras concentrated in non-negative degrees to the category of cosimplicial $R$-algebras. If the DG algebra $A_{R}^{*}$ is flat over $R$, then so is the cosimplicial algebra $\mathrm{D}^{-1}\left(A_{R}^{\bullet}\right)$.

We now let $R=k$ be a finite field of characteristic $p>0$. Fix a DG algebra $A^{\bullet} \in C^{\geq 0}(k)$ over $k$, and let $\mathcal{A}=\mathrm{D}^{-1}\left(A^{\bullet}\right) \in \operatorname{Fun}(\Delta, k)$ be the corresponding cosimplicial algebra. Applying the constructions of Subsection 4.1 pointwise, we obtain DG cosimplicial algebras $\bar{Q}$. $(\mathcal{A}), P$. $(A)$. We want to apply the formalism of Subsection 4.3 to the augmentation map $\bar{Q}$. $(\mathcal{A}) \rightarrow \mathcal{A}$. In order to do this, we have to specify a symmetric tensor abelian category $\mathcal{C}$ and a subcategory $\operatorname{Ac} \subset \mathcal{D}^{b}(\mathcal{C})$. For $A^{\bullet}$, we take $\mathcal{C}=C(k)$, the category of complexes of $k$-vector spaces; Ac $\subset$ $\mathcal{D}^{b}(\mathcal{C})$ is the minimal thick triangulated subcategory such that $A c \cap \mathcal{C}$ consists of acyclic complexes (in other words, if we represent objects in $\mathcal{D}^{b}(\mathcal{C})=\mathcal{D}^{b}(C(k)$ ) by bicomplexes, then $\mathrm{Ac} \subset \mathcal{D}^{b}(\mathcal{C})$ consists of those bicomplexes whose total complex is acyclic). We have $\mathrm{Ho}(\mathcal{C}) \cong \mathcal{D}(k$-Vect $)$. As for $\mathcal{A}$, we note that since $\bar{Q}$. $(\mathcal{A})$ is a DG comsimplicial algebra, $\mathcal{A}$ also has to be treated a DG cosimplicial algebra (placed in degree 0$)$. Thus for $\mathcal{A}$, we let $\mathcal{C}=C^{\leq 0}(\operatorname{Fun}(\Delta, k))$, the category of complexes of cosimplicial $k$-vector spaces concentrated in negative cohomological degrees. By Dold-Kan equivalence, $\mathcal{C}$ is equivalent to the category $C \leq 0, \geq 0(k)$ of bicomplexes of $k$-vector spaces concentrated in the "top-left" quadrant. For any such complex $\mathcal{K}^{\bullet \cdot \bullet}$, we let

$$
\operatorname{Tot}\left(K^{\bullet \cdot \bullet}\right)^{\bullet}=\bigoplus_{l} K^{l, \cdot-l}
$$


be its total complex, and we let $\mathrm{Ac} \subset \mathcal{D}^{b}(\mathcal{C})$ be the kernel of the functor Tot $\circ \mathrm{D}$ : $\mathcal{D}^{b}(\mathcal{C}) \rightarrow \mathcal{D}^{b}(C(k)) \mathcal{D}(k$-Vect $)$. Again, we have $\mathrm{Ho}(\mathcal{C}) \cong \mathcal{D}(k$-Vect $)$. We note that the condition 4.9 is obviously satisfied both for $\mathcal{C}=C(k)$ and for $\mathcal{C}=$ $C^{\leq 0}(\operatorname{Fun}(\Delta, k))$, so that the formalism of Subsection 4.3 applies.

Lemma 5.6. There exists an equivalence of categories $\mathrm{Ho}\left(A^{\bullet}\right.$-bimod $) \cong \mathrm{Ho}(\mathcal{A}$-bimod $)$ which induces an isomorphism

$$
{\overline{H H_{\mathcal{D}}}}_{\dot{\mathcal{B}}}\left(A^{\bullet}\right) \cong \overline{H_{H_{\mathcal{D}}}^{\bullet}}(\mathcal{A}) .
$$

Proof. Let $A^{\bullet}$-bimod ${ }^{\geq 0} \subset A^{\bullet}$-bimod be the full subcategory spannes by DG $A^{\bullet}$ bimodules concentrated in non-negative degrees. Embed $\operatorname{Fun}(\Delta, k)$ into $C^{\geq 0}$ (Fun $(\Delta, k))$ as the subcategory of complexes concentrated in degree 0 . Then the inverse Dold-Kand equivalence $\mathrm{D}^{-1}: C^{\geq 0}(k) \rightarrow \operatorname{Fun}(\Delta, k)$ gives an exact functor $A^{\bullet}$-bimod ${ }^{\geq 0} \rightarrow \mathcal{A}$-bimod, which extends to a functor

$$
\mathrm{D}^{-1}: \mathcal{D}^{b}\left(A^{\bullet} \text {-bimod }{ }^{\geq 0}\right) \rightarrow \mathcal{D}^{b}(\mathcal{A} \text {-bimod }) .
$$

This functor sends Ac $\subset \mathcal{D}^{b}\left(A^{\bullet}\right.$-bimod $\left.{ }^{\geq 0}\right)$ into $\mathrm{Ac} \subset \mathcal{D}^{b}(\mathcal{A}$-bimod $)$, thus descends to a triangulated functor between quotient categories. But the quotient $\mathcal{D}^{b}\left(A^{\bullet}\right.$-bimod $\left.{ }^{\geq 0}\right) /\left(\mathcal{D}^{b}\left(A^{\bullet}\right.\right.$-bimod $\left.\left.{ }^{\geq 0}\right) \cap \mathrm{Ac}\right)$ is the whole $\mathrm{Ho}\left(A^{\bullet}\right.$-bimod $)$, and the quotient $\mathcal{D}^{b}(\mathcal{A}$-bimod $) /\left(\mathcal{D}^{b}(\mathcal{A}\right.$-bimod $\left.) \cap \mathrm{Ac}\right)$ is the whole category $\mathrm{Ho}(\mathcal{A}$-bimod $)$. We obtain a triangulated functor

$$
\mathrm{D}^{-1}: \mathrm{Ho}\left(A^{\bullet} \text {-bimod }\right) \rightarrow \mathrm{Ho}(\mathcal{A} \text {-bimod }) .
$$

Both categories are generated by free bimodules, and since the map (5.1) is a quasiisomorphism for algebras over a field, $\mathrm{D}^{-1}$ sends the free bimodule $A^{\bullet \text { opp }} \otimes$ $M^{\bullet} \otimes \mathcal{A}^{\bullet}$ into an $\mathcal{A}$-bimodule equivalent to $\mathcal{A}^{\text {opp }} \otimes \mathrm{D}^{-1}\left(M^{\bullet}\right) \otimes \mathcal{A}$. Thus $\mathrm{D}^{-1}$ is essentially surjective, and to prove that it is an equivalence, it suffices to prove that the corresponding functor

$$
\mathrm{D}^{-1}: \mathrm{Ho}\left(C^{\geq 0}(k)\right) \rightarrow \mathrm{Ho}\left(C^{\leq 0}(\operatorname{Fun}(\Delta, k))\right.
$$

is an equivalence. This is obvious - both categories are equivalent to the unbounded derived category $\mathcal{D}(k$-Vect $)$.

Now, as explained in Subsection 4.1, to work correctly with the infinite DG algebra $\bar{Q}$. $(\mathcal{A})$, we have to treat it as a filtered DG algebra, with some increasing filtration cofinal to the stupid filtration. This again can be done with the framework of Subsection 4.3, but we have to replace the category of $k$-vector 
spaces with the abelian tensor category $\mathcal{C}_{0}$ graded modules over the polynomial algebra $k[t]$ in one formal variable $t$ of degree 1 . The category $\mathcal{C}_{0}$ is a symmetric tensor abelian category; we take $\mathcal{C}=C^{\leq 0}\left(\operatorname{Fun}\left(\Delta, \mathcal{C}_{0}\right)\right)$, with Ac spanned by those objects whose total complex is acyclic. Again, the condition 4.9 is obviously satisfied. The subcategory of flat objects in $\mathcal{C}_{0}$ is equivalent to the category of filtered $k$-vector spaces - this is the standard Rees construction, - thus a flat DG cosimplicial algebra in $\mathcal{C}_{0}$ is the same as a filtered DG cosimplicial algebra over $k$. We treat $\mathcal{A}$ as such by placing it, as before, in degree 0 , and giving it an increasing filtration $F$. $\mathcal{A}$ by

$$
F_{0} \mathcal{A}=k \cdot 1 \subset \mathcal{A}, \quad F_{1} \mathcal{A}=\mathcal{A} .
$$

We denote the corresponding quotient category of filtered DG cosimplicial $\mathcal{A}$ bimodules by $\mathrm{Ho}_{F}(\mathcal{A}$-bimod $)$. For any $M_{\bullet}, M^{\prime} \in \mathrm{Ho}_{F}(\mathcal{A}$-bimod $)$, we denote by $\operatorname{Ext}_{\mathcal{D F}}(-,-)$ the Ext ${ }^{\circ}$-groups in the category $\operatorname{Ho}_{F}(\mathcal{A}$-bimod $)$, and we denote by $\overline{H H}_{\mathcal{D F}}(\mathcal{A},-)=\operatorname{Ext}{ }^{\bullet}\left(\mathcal{I}_{\mathcal{A}},-\right)$ the quotient Hochschild cohomology $\left(\mathcal{I}_{\mathcal{A}} \subset \mathcal{A}^{\text {opp }} \otimes \mathcal{A}\right.$ inherits the filtration from the free bimodule $\left.\mathcal{A}^{\text {opp }} \otimes \mathcal{A}\right)$. Forgetting the filtration gives a natural functor $\mathrm{Ho}_{F}(\mathcal{A}$-bimod $) \rightarrow \mathrm{Ho}(\mathcal{A}$-bimod $)$ which induces a map

$$
\operatorname{Ext}_{\mathcal{D F}}^{\cdot}\left(M_{\bullet}, M_{\bullet}^{\prime}\right) \rightarrow \operatorname{Ext}_{\mathcal{D}}^{\cdot}\left(M_{\bullet}, M_{\bullet}^{\prime}\right)
$$

for any $M_{\bullet}, M_{\bullet}^{\prime} \in \mathrm{Ho}_{F}(\mathcal{A}$-bimod $)$.

Definition 5.7. A filtered bicomplex $\left\langle K^{\bullet}, \cdot, F\right.$. $\rangle$ is said to be bounded from above, resp. from below by some integer $n$ if for any $m$, the total complex of the associated graded quotient $\operatorname{~gr}_{m}^{F} K^{\bullet} \cdot \boldsymbol{\bullet}$ has no cohomology in degrees $\geq n-m$, resp. $\leq n-m$. A filtered object $K . \in C^{\leq 0}(\operatorname{Fun}(\Delta, k))$ is bounded from above/below by $n$ if so is the filtered bicomplex $\mathrm{D}\left(K_{\text {. }}\right) \in C^{\leq 0, \geq 0}(k)$.

Lemma 5.8. Assume given an object $M_{.} \in \mathrm{Ho}_{F}(\mathcal{A}$-bimod) which is bounded from below by $n$, and an object $M_{\bullet} \in \mathrm{Ho}_{F}(\mathcal{A}$-bimod) which is bounded from above by $n^{\prime}$. Then the natural map

$$
\operatorname{Ext}_{\mathcal{D F}}^{i}\left(M_{\bullet}, M_{\bullet}^{\prime}\right) \rightarrow \operatorname{Ext}_{\mathcal{D}}^{i}\left(M_{\bullet}, M_{\bullet}^{\prime}\right)
$$

is an isomorphism for all $i \geq n-n^{\prime}$. In particular, for any $M_{\bullet} \in \mathrm{Ho}_{F}(\mathcal{A})$ bounded from above by 0 , the map

$$
\overline{H H}_{\mathcal{D F}}^{i}\left(\mathcal{A}, M_{\bullet}\right) \rightarrow \overline{H H}_{\mathcal{D}}^{i}\left(\mathcal{A}, M_{\bullet}\right)
$$

is an isomorphism for any $i \geq 0$. 
Proof. By dévissage, it suffices to consider those $M_{\text {. }}^{\prime}$ which have only one nontrivial graded piece with respect to the filtration, say $\mathrm{gr}_{m^{\prime}}^{F} M_{\text {. }}^{\prime}$. Let $m$ be smallest integer such that $F_{m} M_{\bullet} \neq 0$. If $m \geq m^{\prime},(5.3)$ is obvious (and holds for all $i$ ). Assume $m<m^{\prime}$. In the degenerate case $\mathcal{A}=k$, the left-hand side of (5.3) is 0 , while the right-hand side is 0 if $i>n-n^{\prime}$, so that the claim holds for $\mathcal{A}=k$. Then by adjunction, it holds for any $\mathcal{A}$ as above when $M_{\bullet}=\mathcal{A}^{\text {opp }} \otimes V_{\bullet} \otimes \mathcal{A}$ is the free bimodule generated by some filtered DG cosimplicial $V$. bounded from below by $n$. To finish the proof of the first claim, we use the descending induction on $m<m^{\prime}$. For any $M_{\bullet}$, we have a canonical multiplication map $\mu: \mathcal{A}^{\text {opp }} \otimes M_{\bullet} \otimes \mathcal{A} \rightarrow M$. which is a weak equivalence on $F_{m}$ and surjective on the cohomology of the total complex of each graded piece $\operatorname{gr}_{l}^{F}, l \geq m+1$. Therefore if we form the filtered exact triangle

$$
\widetilde{M} \cdot \longrightarrow \mathcal{A}^{\text {opp }} \otimes M_{\bullet} \otimes \mathcal{A} \stackrel{\mu}{\longrightarrow} M_{\bullet} \longrightarrow,
$$

then $F_{l} \widetilde{M}$. $=0$ for $l<m+1$, and moreover, $\widetilde{M}$. is bounded from below by $n+1$. To deduce the induction step, apply dévissage. The second claim follows immediately, since the $\mathcal{A}$-bimodule $\mathcal{I}_{\mathcal{A}}$ is obviously bounded from below by 0 .

Lemma 5.9. Let $\mathcal{V} \in \operatorname{Fun}(\Delta, k)$ be such that the complex $\mathrm{D}(\mathcal{V}) \in C^{\geq 0}(k)$ has no cohomology in degrees $\geq n$ for some integer $n$. Then the $D G$ cosimplicial $k$-vector space $\bar{Q} .(\mathcal{V})$ equipped with the stupid filtration is bounded from above by pn in the sense of Definition 5.7. Moreover, the same is true for its associated graded pieces $\operatorname{gr} \bar{Q}^{\circ}(\mathcal{V})$ with respect to the canonical filtration.

Proof. By Definition 5.7, what we have to prove is that for every $m \geq 0$, the cosimplicial vector space $\bar{Q}_{m}(\mathcal{V})$ goes under the Dold-Kan equivalence to a complex which has no cohomology in degrees $\geq p n$, and moreover, the same is true for the image $\operatorname{Im} d_{m}^{\mathcal{V}}$ of the differential $d_{m}^{\mathcal{V}}: \bar{Q}_{m+1}(\mathcal{V}) \rightarrow \bar{Q}_{m}(\mathcal{V})$. If $\mathrm{D}(\mathcal{V})$ is entirely concentrated in degrees $\leq n$, this is clear: indeed, by (3.18), for any $m \geq 1$ the complex $\bar{Q}_{m}(\mathcal{V})$ is a direct summand of

$$
\bar{Q}_{m}^{\prime}(\mathcal{V})=\left(\operatorname{Span}_{k}(\mathcal{V} \otimes W)\right)_{k^{*}}
$$

where $W$ is a $2^{m}$-dimensional $k$-vector space, and since char $k=p$, the right-hand side goes under the Dold-Kan equivalence into a complex concentrated in degrees $\leq p n$. 
To deduce the general case, it suffices to prove that a map $\rho: \mathcal{V}^{\prime} \rightarrow \mathcal{V}^{\prime \prime}$ between $\mathcal{V}^{\prime}, \mathcal{V}^{\prime \prime} \in \operatorname{Fun}(\Delta, k)$ such that $\mathrm{D}(\rho)$ is a quasiisomorphism induces maps

$$
\mathrm{D}\left(\bar{Q}_{m}\left(\mathcal{V}^{\prime}\right)\right) \rightarrow \mathrm{D}\left(\bar{Q}_{m}\left(\mathcal{V}^{\prime \prime}\right)\right), \quad \mathrm{D}\left(\operatorname{Im} d_{m}^{\mathcal{V}^{\prime}}\right) \rightarrow \mathrm{D}\left(\operatorname{Im} d_{m}^{\mathcal{V}^{\prime \prime}}\right),
$$

which are quasiisomorphisms for every $m \geq 0$. For the first map, this is clear from (5.4), since the functor $\left(\operatorname{Span}_{k}(-)\right)_{k^{*}}$ preserves quasiisomorphism. For the second map, it suffices by induction to prove that the induced map

$$
\mathrm{D}\left(H_{m}\left(\bar{Q} .\left(\mathcal{V}^{\prime}\right)\right)\right) \rightarrow \mathrm{D}\left(H_{m}\left(\bar{Q} .\left(\mathcal{V}^{\prime \prime}\right)\right)\right)
$$

on homology is a quasiisomorphism for every $m$; this is again clear, since $H_{m}(\bar{Q} .(\mathcal{V}))$ $\cong \mathcal{V} \otimes H_{m}(\bar{Q} .(k))$.

Proposition 5.10. Assume given a DG algebra $A^{\bullet}$ over a finite field $k$ of characteristic $p>0$, and assume that

(i) $A^{\bullet}$ lifts to a flat DG algebra over the Witt vectors ring $W_{2}(k)$, and

(ii) $\overline{H H}_{\mathcal{D}}^{i}\left(A^{\bullet}\right)=0$ whenever $i \geq 2 p$.

Moreover, assume that $A^{\bullet}$ has no cohomology in degrees $\geq n$ for some integer $n \geq 0$. Then the augmentation map $\bar{Q} .(\mathcal{A}) \rightarrow \mathcal{A}$ for the cosimplicial k-algebra $\mathcal{A}=\mathrm{D}^{-1}\left(A^{\bullet}\right)$ admits a weak splitting $\mathcal{A}_{\bullet}, s: \mathcal{A} . \rightarrow \bar{Q} .(\mathcal{A})$.

Proof. Consider the quotients $\bar{Q}_{\leq}$. $(\mathcal{A})$ of the canonical filtration on $\bar{Q}$. $(\mathcal{A})$, and equip them with the stupid filtration. By Lemma 5.9, we may assume that they are all bounded from above by $N$ in the sense of Definition 5.7 for some fixed integer $N$. Redefine the filtration on $\bar{Q}$. $(\mathcal{A})$ by

$$
F_{0} \bar{Q} .(\mathcal{A})=k \cdot 1 \subset \mathcal{A}, \quad F_{l} \bar{Q}_{m}(\mathcal{A})= \begin{cases}0, & l \geq 1, m>l+N \\ \bar{Q}_{m}(\mathcal{A}), & l \geq 1, m \leq l+N\end{cases}
$$

and redefine the filtration on the quotients $\bar{Q}_{\leq \bullet}(\mathcal{A})$ accordingly.

Since $A^{\bullet}$ lifts to a flat algebra over $W_{2}(k)$, so does $\mathcal{A}$. By Corollary 4.12, fixing such a lifting defines a DG splitting of the projection $\bar{Q}_{\leq 1}(\mathcal{A}([n])) \rightarrow \mathcal{A}([n])$ for every $[n] \in \Delta$ which, moreover, depends on $[n]$ in a functorial way. Thus we have a DG splitting $\left\langle\mathcal{A}_{\bullet}^{1}, s\right\rangle$ of the augmentation projection $\bar{Q}_{\leq 1}(\mathcal{A}) \rightarrow \mathcal{A}$. Define a filtration on $\mathcal{A}_{\text {. by }}^{1} F_{0} \mathcal{A}_{\text {• }}^{1}=k \cdot 1, F_{1} \mathcal{A}_{\text {. }}^{1}=\mathcal{A}_{\text {: }}^{1}$; then this DG splitting is also a weak splitting of the map $\bar{Q}_{\leq 1}(\mathcal{A}) \rightarrow \mathcal{A}$ of filtered DG cosimplicial algebras. 
By induction, we extend it to a compatible system $\mathcal{A}_{\text {. }}^{i}$ of weak splittings of the projections $\bar{Q}_{\leq i}(\mathcal{A}) \rightarrow \mathcal{A}$. Namely, assume given such a splitting $\mathcal{A}_{\bullet}^{i-1}, s: \mathcal{A}_{\bullet}^{i-1} \rightarrow$ $\bar{Q}_{\leq i-1}(\mathcal{A})$. Let $\widetilde{\mathcal{A}}_{\bullet}^{i}=\mathcal{A}_{\bullet}^{i-1} \oplus_{\bar{Q}_{\leq i-1}(\mathcal{A})} \bar{Q}_{\leq i}(\mathcal{A})$, as in the proof of Corollary 4.12. Then $\widetilde{A}_{\text {. }}^{i}$ is a square-zero extension of $\mathcal{A}_{\bullet}^{i-1}$ by $\operatorname{gr}^{i} \bar{Q} .(\mathcal{A})$, the $i$-th associated graded quotient with respect to the canonical filtration. But $A_{\bullet}^{i-1}$ is weakly equivalent to $\mathcal{A}_{\bullet}^{1}$, which is in turn weakly equivalent to $\mathcal{A}$; by Lemma 5.8 , we have

$$
\overline{H H}_{\mathcal{D F}}^{2}\left(\mathcal{A}_{\bullet}^{i-1}, \operatorname{gr}^{i} \bar{Q} .(\mathcal{A})\right) \cong \overline{H H}_{\mathcal{D F}}^{2}\left(\mathcal{A}_{\bullet}^{1}, \operatorname{gr}^{i} \bar{Q} .(\mathcal{A})\right) \cong \overline{H H}_{\mathcal{D}}^{2}\left(\mathcal{A}_{\bullet}^{1}, \operatorname{gr}^{i} \bar{Q}_{\bullet}(\mathcal{A})\right),
$$

and by $(4.1), \operatorname{gr}^{i} \bar{Q}$. $(\mathcal{A})$ is quasiisomorphic to $\operatorname{gr}^{i} \bar{Q} .(k) \otimes \mathcal{A}$, hence acyclic when $2 \leq i \leq 2 p-3$. Then by $(5.2)$ we have

$$
\overline{H H}_{\mathcal{D}}^{2}\left(\mathcal{A}_{\bullet}^{i-1}, \operatorname{gr}^{i} \bar{Q} .(\mathcal{A})\right) \cong \overline{H H}^{i+2}(\mathcal{A}) \otimes \operatorname{gr}^{i} \bar{Q} .(k) \cong 0
$$

for $2 \leq i \leq 2 p-3$, and for $i \geq 2 p-2$, this equality follows from the assumption (ii). By Lemma 4.17, we conclude that the projection $\widetilde{\mathcal{A}}^{i} \rightarrow \mathcal{A}^{i-1}$ admits a weak splitting $s_{i}: \mathcal{A}_{\bullet}^{i} \rightarrow \widetilde{\mathcal{A}}_{\text {. }}^{i}$. Composing it with the projection $\widetilde{\mathcal{A}}_{\bullet}^{i} \rightarrow \bar{Q}_{\leq i}(\mathcal{A})$, we obtain a weak splitting for $\bar{Q}_{\leq i}(\mathcal{A}) \rightarrow \mathcal{A}$.

We note that for any integers $i \geq 0$ and $l<i-N$, we obviously have $F_{l} \operatorname{gr}^{i} \bar{Q} .(\mathcal{A})=0$, so that the projection $F_{l} \widetilde{A}^{i} \rightarrow F_{l} \mathcal{A}_{\text {. }}^{i-1}$ is not just a quasiisomorphism but an actual isomorphism. Replacing $\mathcal{A}_{\text {. }}^{i}$ with $\mathcal{A}_{\bullet}^{i} / F_{i-N}\left(\operatorname{Ker} s_{i}\right)$, we can arrange so that the composition map $F_{l} \mathcal{A}_{\text {. }}^{i} \rightarrow F_{l} \mathcal{A}_{\text {. }}^{i-1}$ also is an isomorphism when $i>l+N$. We now let

$$
\mathcal{A}_{.}=\lim _{\rightarrow} \lim _{\leftarrow} F_{l} A_{\text {. }}^{i}
$$

This is a DG cosimplicial algebra; by construction, it comes equipped with a map $\mathcal{A}$. $\rightarrow \bar{Q}$. $(\mathcal{A})$. Moreover, for every $l$ the projective system $F_{l} A_{\text {. }}^{i}$ of filtered weak equivalences $F_{l} A_{\text {. }}^{i} \rightarrow F_{l} A_{.}^{i-1}$ stabilizes at a finite level. Therefore the composition map $\mathcal{A}$. $\rightarrow \mathcal{A}$ is also a filtered weak equivalence, and $\mathcal{A}$. is the required weak splitting.

Our proof of Theorem 5.5 would become simpler if we could improve Proposition 5.10 so as to to provide a DG splitting, not just a weak splitting. Unfortunately, this can be done only if we forget the multiplicative structures.

Lemma 5.11. Assume given a cosimplicial $k$-vector space $\mathcal{V} \in \operatorname{Fun}(\Delta, k)$, which we treat as an object of the abelian category $C^{\leq 0}(\operatorname{Fun}(\Delta, k))$ by placing it in degree 
0 , and a complex $\mathcal{V} . \in C^{\leq 0}(\operatorname{Fun}(\Delta, k))$. Then any surjective weak equivalence $s: \mathcal{V} . \rightarrow \mathcal{V}$ admits a $D G$ splitting $\overline{\mathcal{V}} ., \overline{\mathcal{V}}_{.} \rightarrow \mathcal{V}$

Proof. Apply the Dold-Kan equivalence to obtain a complex $V^{\bullet}=\mathrm{D}(\mathcal{V})$, a bicomplex $V^{\bullet} \cdot \boldsymbol{D}\left(\mathcal{V}_{\bullet}\right)$, and a surjective map $s: V^{\bullet \cdot \bullet} \rightarrow V^{\bullet}$. We have to prove that $s$ splits after passing to $\mathcal{D}^{\leq 0}\left(C^{\geq 0}(k\right.$-Vect $\left.)\right)$. Denote by $W^{\bullet \cdot \bullet} \subset V^{\bullet \bullet}$ the kernel of the map $s$. Since $s$ is a surjective weak equivalence, the total complex $\operatorname{Tot}\left(W^{\bullet \cdot \bullet}\right)=\operatorname{Ker} \operatorname{Tot}(s) \in \mathcal{D}(k$-Vect $)$ is acyclic, and it suffices to prove that for any $K^{\bullet} \in C^{\geq 0}(k), K^{\bullet} \cdot \bullet D^{\leq 0}\left(C^{\leq 0}(k\right.$-Vect $\left.)\right)$, the natural map

$$
\operatorname{RHom}^{i}\left(K^{\bullet}, K^{\bullet \bullet}\right) \rightarrow \operatorname{RHom}^{i}\left(K^{\bullet}, \operatorname{Tot}\left(K^{\bullet} \bullet\right)\right)
$$

is an isomorphism for $i=1$. But this map is actually an isomorphism for any $i \geq 1$; to prove it, one can apply dévissage, and then it suffices to check the claim for $K^{\bullet}$ concentrated in a single degree and $K^{\bullet \bullet}$ concentrated in a single bidegree, which is a trivial computation.

5.3. Proofs. We now turn to the proof of Theorem 5.5. First, fix a DG algebra $A^{\bullet} \in C^{\geq 0}(k)$ over a finite field $k$ of arbitrary characteristic.

Lemma 5.12. If $A^{\cdot}$ is smooth, then the object $A_{\sharp} \in \mathcal{D}(\Lambda, k)$ and the objects $i^{*} A_{\sharp}^{\cdot}, \pi^{*} A_{\sharp}^{*} \in \mathcal{D}\left(\Lambda_{p}, k\right)$ are small in the sense of Definition 1.18 .

Proof. As in Lemma 2.9, it suffices to prove that the bar resolution $C$. $\left(A^{\bullet}\right)$ is effectively finite in the category $\mathcal{D}\left(\operatorname{Fun}\left(\Delta^{o}, A^{\bullet}\right.\right.$-bimod $\left.)\right)$ of simplicial $A^{\circ}$-bimodules. Every term $F_{i} C \cdot\left(A^{\bullet}\right)$ of the stupid filtration is by definition a perfect $A^{\bullet}$-bimodule. Since $A^{\bullet}$ is smooth, so is the diagonal bimodule $A^{\bullet}$. Thus for any $m \geq 1$, $\tau_{m}\left(C \cdot\left(A^{\bullet}\right)\right)$ is a perfect object of $\mathcal{D} \mathrm{F}_{[0,1]}\left(A^{\bullet}\right)$. Therefore the quasiisomorphism

$$
\tau_{m}\left(C \cdot\left(A^{\bullet}\right)\right) \cong \lim _{\substack{m^{\prime} \\ \rightarrow}} \tau_{m}\left(F_{m^{\prime}} C \cdot\left(A^{\bullet}\right)\right)
$$

factors through some $\tau_{m}\left(F_{m^{\prime}} C \cdot\left(A^{\bullet}\right)\right)$, and we are done by Lemma 1.4.

Next, we need a cosimplicial version of Proposition 4.7. For any associative unital cosimplicial $k$-algebra $\mathcal{A} \in \operatorname{Fun}(\Delta, k)$, we can apply the $\sharp$-construction pointwise and obtain an object $\mathcal{A}_{\sharp} \in \operatorname{Fun}(\Lambda \times \Delta, k)$; for a DG cosimplicial algebra $\mathcal{A}_{\text {. }}$, we obtain an object $\mathcal{A}_{\bullet \sharp} \in D(\Lambda \times \Delta, k)$. Let $\tau: \Lambda \times \Delta \rightarrow \Lambda$ be the natural projection. 
Definition 5.13. The Hochschild, cyclic and periodic cyclic homology of a cosimplicial algebra $\mathcal{A} \in \operatorname{Fun}(\Delta, k)$ are given by

$$
\begin{aligned}
& H H_{.}(\mathcal{A})=H H_{\bullet}\left(R^{\bullet} \tau_{*} \mathcal{A}_{\sharp}\right), \\
& H C .(\mathcal{A})=H C .\left(R^{\circ} \tau_{*} \mathcal{A}_{\sharp}\right), \\
& H P .(\mathcal{A})=H P_{.}\left(R^{\bullet} \tau_{*} \mathcal{A}_{\sharp}\right) .
\end{aligned}
$$

The Hochschild, cyclic and periodic cyclic homology of a DG cosimplicial $k$ algebra $\mathcal{A}$. is given by

$$
\begin{aligned}
& H H_{\bullet}\left(\mathcal{A}_{\bullet}\right)=\lim _{\rightarrow} H H_{\bullet}\left(R^{\bullet} \tau_{*} F_{i} \mathcal{A}_{\bullet \sharp}\right), \\
& H C ._{\bullet}\left(\mathcal{A}_{\bullet}\right)=\lim _{\rightarrow} H C_{\bullet}\left(R^{\bullet} \tau_{*} F_{i} \mathcal{A}_{\bullet}\right), \\
& H P_{\bullet}\left(\mathcal{A}_{\bullet}\right)=\lim _{\rightarrow} H P_{\bullet}\left(R^{\bullet} \tau_{*} F_{i} \mathcal{A}_{\bullet} \sharp,\right.
\end{aligned}
$$

where $F_{i} \mathcal{A}_{\bullet \sharp}$ is the stupid filtration.

We note that by this definition, a weak equivalence $\mathcal{A}$. $\rightarrow \mathcal{A}_{\text {。 }}^{\prime}$ of DG cosimplicial algebras induces an isomorphism on Hochschild and cyclic homology (and on periodic cyclic homology, if both DG algebras are concentrated in a finite range of degrees). We also note that since the map (5.1) is a quasiisomorphism for flat algeras, we have

$$
H_{\bullet}(\mathcal{A}) \cong H_{\bullet}\left(A^{\bullet}\right), \quad H C \cdot(\mathcal{A}) \cong H C_{\bullet}\left(A^{\bullet}\right), \quad H P .(\mathcal{A}) \cong H P_{.}\left(A^{\bullet}\right)
$$

for any DG $k$-algebra $A^{\bullet}$ and the cosimplicial $k$-algebra $\mathcal{A}=\mathrm{D}^{-1}\left(A^{\bullet}\right)$.

Proposition 5.14. Assume that a DG algebra $A^{\bullet}$ over $k$ is smooth, and that the augmentation map $\bar{Q}$. $(\mathcal{A}) \rightarrow \mathcal{A}$ for the corresponding cosimplicial algebra $\mathcal{A}=\mathrm{D}^{-1}\left(A^{\bullet}\right)$ admits a weak splitting $\langle\mathcal{A} ., s\rangle$. Moreover, assume that $A^{\bullet}$ has no cohomology in degrees $\geq q$ for some integer $q \geq 0$. Then the map $s \circ \varphi: \mathcal{A}$. $\rightarrow$ P. $(\mathcal{A})$ induces an isomorphism

$$
H H_{\bullet}\left(\mathcal{A}_{\bullet}^{(1)}\right)((u)) \stackrel{\sim}{\longrightarrow} H P .(\mathcal{A}) .
$$

Proof. By Lemma 5.12, both $R^{\bullet} \tau_{*} \mathcal{A}_{\bullet}$ and $R^{\bullet} \tau_{*} i^{*} \mathcal{A}_{\sharp}$ are small in $\mathcal{D}\left(\Lambda_{p}, k\right)$ in the sense of Definition 1.18; as in Lemma 4.3, the same can be said about every finite filtered piece $R^{\bullet} \tau_{*} F_{n} P .(\mathcal{A})_{\sharp}$. Then as in the proof of Proposition 2.10, it suffices to prove that for any $n$, the map $(s \circ \varphi)^{\otimes n}$ induces an isomorphism

$$
\check{H}_{.}\left(\mathbb{Z} / p \mathbb{Z}, R^{\bullet} \tau_{*} \mathcal{A}_{\bullet}^{\otimes n}\right)^{(1)} \cong \check{H}_{\bullet}\left(\mathbb{Z} / p \mathbb{Z}, R^{\bullet} \tau_{*} P(\mathcal{A})_{\cdot}^{\otimes n}\right) .
$$


From now on, we can forget about the algebra structure on $\mathcal{A}$. and treat it as a complex of cosimplicial $k$-vector spaces. Then we can apply Lemma 5.11 to the weak equivalence $\mathcal{A}$. $\rightarrow \mathcal{A}$ and replace $\mathcal{A}$. with $\overline{\mathcal{A}}_{\mathbf{0}}$. In other words, we can assume that $s: \mathcal{A} . \rightarrow \bar{Q}$. $(\mathcal{A})$ is in fact a DG splitting, and $\mathcal{A}$. $([m]) \rightarrow \mathcal{A}([m])$ is a quasiisomorphism for any $[m] \in \Delta$. Since $\mathcal{A}$ by assumption has no cohomology in degrees $\gg 0$, it is effectively finite as a cosimplicial $k$-vector space. Then its tensor power $P_{0}(\mathcal{A})^{\otimes n}=\mathcal{A}^{\otimes p n} \in \operatorname{Fun}(\Delta, k[\mathbb{Z} / p \mathbb{Z}] \bmod )$ is effectively finite by Corollary 1.5 (ii), and so is the stupid filtration term $F_{i} P .(\mathcal{A})^{\otimes n}=P_{0}(\mathcal{A})^{\otimes n} \otimes$ $F_{i}(P .(k))$ for any $i \geq 1$. Then as in the proof of Lemma 1.19, we can remove $R^{\circ} \tau_{*}$ - it suffices to prove that the map $(s \circ \varphi)^{\otimes n}$ induces an isomorphism

$$
\check{H}_{.}\left(\mathbb{Z} / p \mathbb{Z}, \mathcal{A}_{\bullet}^{\otimes n}([m])\right)^{(1)} \cong \check{H}_{.}\left(\mathbb{Z} / p \mathbb{Z}, P(\mathcal{A})_{\bullet}^{\otimes n}([m])\right)
$$

for any $n, m$. This is Lemma 4.6.

Proposition 5.15. Assume given a smooth DG algebra $A^{\bullet}$ over a finite field $k$ of characteristic $p>0$ which is concentrated in non-negative degrees. Assume in addition that $A^{\bullet}$ satisfies the assumptions (i), (ii) of Proposition 5.10, and that $A^{\bullet}$ has no cohomology in degrees $\geq q$ for some integer $q \geq 0$. Then there exists an isomorphism

$$
H H_{\bullet}\left(A^{\bullet}\right)^{(1)}((u)) \cong H P \cdot\left(A^{\bullet}\right)
$$

Moreover, assume that $A^{\bullet}$ is compact. Then the Hodge-to-de Rham spectral sequence $H_{\mathbf{*}}\left(A^{\bullet}\right)\left[u^{-1}\right] \Rightarrow H C .\left(A^{*}\right)$ degenerates at first term.

Proof. Let $\mathcal{A}=\mathrm{D}^{-1}\left(\mathcal{A}^{\bullet}\right) \in \operatorname{Fun}(\Delta, k)$. By Proposition 5.10, the augmentation map $\bar{Q} .(\mathcal{A}) \rightarrow \mathcal{A}$ admits a weak splitting; then Proposition 5.14 gives the required isomorphism

$$
H_{\bullet} \cdot\left(A^{\bullet}\right)^{(1)}((u)) \cong H_{\bullet}\left(\mathcal{A}^{(1)}\right)((u)) \cong H_{\bullet}(\mathcal{A}) \cong H P .\left(A^{\bullet}\right) .
$$

Since $A^{\bullet}$ is smooth and compact, the groups $H H_{i}\left(A^{\bullet}\right), H C_{i}\left(A^{\bullet}\right), H P_{i}\left(A^{\bullet}\right)$ are finite-dimensional $k$-vector spaces for every $i$, and we have $H H_{i}\left(A^{\bullet}\right)=0, H C_{i}\left(A^{\bullet}\right)$ $=H P_{i}\left(A^{\bullet}\right)$ for $i \gg 0$. Thus for $i \gg 0$ we have

$$
\operatorname{dim}_{k} H C_{i}\left(A^{\bullet}\right)=\sum_{j} \operatorname{dim}_{k} H H_{i-2 j}\left(A^{\bullet}\right)^{(1)}=\sum_{j} \operatorname{dim}_{k} H H_{i-2 j}\left(A^{\bullet}\right),
$$

and the Hodge-to-de Rham spectral sequence degenerates in this range of degrees by the standard degeneration criterion [D2]. Since all the differentials in the sequence commute with multiplication by $u$, it must degenerate everywhere. 
Proof of Theorem 5.5. We assume given a smooth and compact DG algebra $A^{\bullet}$ over a field $K$ of characteristic 0 which is concentrated in non-negative degrees. By Theorem 5.4, we may assume that there exists a subring $R \subset K$ which is finitely generated over $\mathbb{Z}$ and a DG algebra $A_{R}^{\cdot}$ such that $A_{R}^{*} \otimes_{R} K$ is quasiisomorphic to $A^{\bullet}$; moreover, $A_{R}^{*}$ is saturated over $R$. Replacing $A_{R}^{*}$ with a quasiisomorphic algebra if necessary, we may in addition assume that $A_{R}^{i}=0$ for negative $i$, and that every $A_{R}^{i}$ is a flat $R$-module. Since $A_{R}^{*}$ is compact, we have $\overline{H H}_{\mathcal{D}}^{i}\left(A_{R}^{\cdot}\right)=0$ for $i \geq n$, so some constant $n$. Since $R$ is of finite type over $\mathbb{Z}$, the residue field $k=R / \mathfrak{m}$ for any maximal ideal $\mathfrak{m} \subset R$ is a finite field, of some positive characteristic $p>0$. Replacing $R$ with its localization $R^{\prime}$, and $\mathcal{A}_{R}$ with $\mathcal{A}_{R^{\prime}}=\mathcal{A}_{R} \otimes_{R} R^{\prime}$, we may assume that for any $\mathfrak{m} \subset R^{\prime}, p=\operatorname{char} R^{\prime} / \mathfrak{m}$ is non-trivial in $\mathfrak{m} / \mathfrak{m}^{2}$ (in other words, $p$ is unramified in $R^{\prime}$ ), and $2 p>n$, so that

$$
\overline{H H}^{i}\left(\dot{A}_{R^{\prime}}\right)=0
$$

whenever $i \geq 2 p$. Then for any $\mathfrak{m} \subset R^{\prime}$, the DG algebra $A_{k}^{\bullet}=A_{R^{\prime}}^{\bullet} \otimes_{R^{\prime}} k$ satisfies the assumptions of Proposition 5.15, so that the Hodge-to-de Rham spectral sequence for $A_{k}^{*}$ degenerates. We conclude that the differentials in the Hodge-tode Rham spectral sequence for $\mathcal{A}_{R^{\prime}}$ are equal to 0 modulo every maximal ideal $\mathfrak{m} \subset R^{\prime}$. Since $A_{R^{\prime}}^{\cdot}$ is compact, $H H_{\bullet}\left(A_{R^{\prime}}^{*}\right)$ is a perfect complex of $R^{\prime}$-modules; this means, by Nakayama, that the spectral sequence degenerates already for $\mathcal{A}_{R^{\prime}}$, and certainly for $\mathcal{A}$.

\section{REFERENCES}

[A1] J.F. Adams, Stable Homotopy and Generalized Homology, Univ. of Chicago Press, 1974. [A2] J.F. Adams, Infinite Loop Spaces, Princeton Univ. Press and Univ. of Tokyo Press, Princeton, NJ, 1978.

[BBD] A. Beilinson, J. Bernstein and P. Deligne, Faisceaux Pervers, Astérisque 100, Soc. Math de France, 1983.

[BGS] A. Beilinson, V. Ginzburg, and V. Schechtman, Koszul duality, J. Geom. Phys. 5 (1988), 317-350.

[B] M. Bökstedt, Topological Hochschild homology, preprint, Bielefeld, 1985.

[BHM] M. Bökstedt, W.C. Hsiang, and I. Madsen, The cyclotomic trace and algebraic K-theory of spaces, Inv. Math. 111 (1993), 465-540.

[Br] L. Breen, Extensions du groupe additif, Publ. Math. IHES 48 (1978), 39-125.

[Co] A. Connes, Cohomologie cyclique et foncteur Ext $^{n}$, Comptes Rendues Ac. Sci. Paris Sér. A-B, 296 (1983), 953-958. 
[D1] P. Deligne, Cohomologie á support propre et construction du foncteur $f^{!}$, appendix to R. Hartshorne, Residues and Duality, Lecture Note in Math. 20, Springer-Verlag, Heidelberg 1966.

[D2] P. Deligne, Théorème de Lefschetz et critères de dégénérescence de suites spectrales, Publ. Math. IHES 35 (1968), 259-278.

[DI] P. Deligne and L. Illusie, Relévements modulo $p^{2}$ et dcomposition du complexe de de Rham, Inv. Math. 89 (1987), 247-270.

[Do] A. Dold, Homology of symmetric products and other functors of complexes, Ann. of Math. 68 (1958), 54-80.

[EM] S. Eilenberg and S. MacLane, Homology theories for multiplicative systems, Trans. AMS 71 (1951), 294-330.

[EKMM] A.D. Elmendorf, I. Kriz, M.A. Mandell, and J.P. May, Rings, modules, and algebras in stable homotopy theory, Mathematical Surveys and Monographs, 47, AMS, Providence, RI, 1997.

[FT1] B. Feigin and B. Tsygan, Cohomology of the Lie algebra of generalized Jacobi matrices, in Russian, Funct. An. Appl. 17 (1983), 86-87.

[FT2] B. Feigin and B. Tsygan, Additive K-Theory, in Lecture Notes in Math. 1289 (1987), 97-209.

[FPSVW] Z. Fiedorowicz, T. Pirashvili, R. Schwänzl, R. Vogt, and F. Waldhausen, Mac Lane homology and topological Hochschild homology, Math. Ann. 303 (1995), 149-164.

[GM] S. Gelfand and Yu. Manin, Methods of homological algebra, Nauka Publishers, Moscow, 1988 (in Russian).

[G] A. Grothendieck, SGA I: Revêtements étales et groupe fondamental, reprinted as Documents Mathématiques 3, SMF, 2003.

$[\mathrm{H}]$ L. Hesselholt, Witt vectors of non-commutative rings and topological cyclic homology, Acta Math. 178 (1997), 109-141.

[HM] L. Hesselholt and I. Madsen, On the K-theory of finite algebras over Witt vectors of perfect fields, Topology 36 (1997), 29-101.

[HKR] G. Hochschild, B. Kostant, and A. Rosenberg, Differential forms on regular affine algebras, Trans. AMS 102 (1962), 383-408.

[HSS] M. Hovey, B. Shipley, and J. Smith, Symmetric spectra, J. AMS 13 (2000), 149-208.

[JP] M. Jibladze and T. Pirashvili, Cohomology of algebraic theories, J. of Algebra, 137 (1991), 253-296.

[Ka] D. Kaledin, Non-commutative Cartier operator and Hodge-to-de Rham degeneration, preprint math.AG/0511665.

[Ke] B. Keller, On differential graded categories, a talk at ICM 2006, Madrid.

[KS] M. Kontsevich and Y. Soibelman, Notes on A-infinity algebras, A-infinity categories and non-commutative geometry, I, preprint math.RA/0606241.

[L] J.-L. Loday, Cyclic Homology, second ed., Springer, 1998.

[LQ] J.-L. Loday and D. Quillen, Homologie cyclique et homologie de l'algèbre de Lie des matrices, Comptes Rendues Ac. Sci. Paris Sér. A-B, 296 (1983), 295-297.

[LP] J.-L. Loday and T. Pirashvili, Chapter 13 in [L]. 
[M] I. Madsen, The cyclotomic trace in algebraic K-theory, First European Congress of Mathematics, Vol. II (Paris, 1992), 213-241, Progr. Math. 120, Birkhäuser, Basel, 1994.

[Mc] S. MacLane, Homologie des anneaux et des modules, Coll. topologie algébrique, Louvain, 1956; 55-80.

[PW] T. Pirashvili and F. Waldhausen, Mac Lane homology and topological Hochschild homology, J. Pure Appl. Alg. 82 (1992), 81-99.

[Se] G. Segal, Categories and cohomology theories, Topology 13 (1974), 293-312.

[T] B. Toën, Rings of definition of smooth and proper dg-algebras, preprint math.AT/0611546.

[TV] B. Toën and M. Vaquié, Moduli of objects in dg-categories, preprint math.AG/0503269.

Dmitry Kaledin

Steklov Math Institute, Moscow, USSR

E-mail: kaledin@mccme.ru 